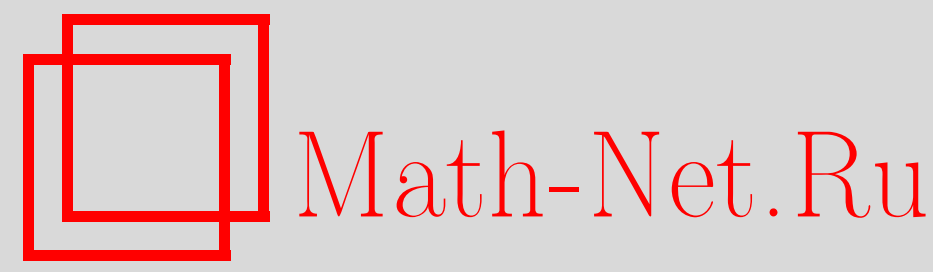

В. В. Зудилин, Тэта-константы и дифференциальные уравнения, Матем. сб., 2000, том 191, номер 12, 77-122

DOI: https://doi.org/10.4213/sm530

Использование Общероссийского математического портала Math-Net.Ru подразумевает, что вы прочитали и согласны с пользовательским соглашением

http: //www.mathnet.ru/rus/agreement

Параметры загрузки:

IP : 52.205 .19 .152

26 апреля 2023 г., 11:06:02 
УДК $511.334+517.953$

\title{
В.В. Зудилин
}

\section{Тэта-константы и дифференциальные уравнения}

\begin{abstract}
Замкнутость тэта-констант (и зигелевых модулярных функций) относительно дифференцирования в одномерном случае хорошо известна. В работе показывается, что тэта-константы и их всевозможные логарифмические производные удовлетворяют нелинейной системе дифференциальных уравнений; ранее была известна только одномерная версия этого результата. Приводится несколько различных примеров подобных нелинейных систем и формулируется теорема о степени трансцендентности дифференциального замыкания поля, порожденного всеми тэта-константами. Исследование модулярных свойств логарифмических производных тэта-констант позволяет получить неизвестные ранее соотношения, связывающие эти функции с самими тэта-константами в размерностях 2 и 3 .

Библиографияя: 26 названий.
\end{abstract}

\section{Введение}

Тэта-функции - насыщенный по красоте классический раздел математики. Их появлению на свет обязаны многие результаты математического анализа, алгебраической геометрии, теории дифференциальных уравнений и других дисциплин математики. Тэта-функциям посвящено большое количество монографий (достойные примеры - книги [1]-[3]) и статей, малую часть которых можно найти в приведенном нами списке литературы.

Первое систематическое изучение одномерных тэта-функций было проведено в работах К. Якоби (см. [4], [5]), правда, его обозначения несколько отличались от появившихся позднее в работах Г. Фробениуса, А. Крацера, В. Виртингера и других:

$$
\begin{aligned}
\vartheta_{1}(z, q) & =2 \sum_{n=0}^{\infty}(-1)^{n} q^{(n+1 / 2)^{2}} \sin (2 n+1) z \\
& =2 q^{1 / 4} \sin z-2 q^{9 / 4} \sin 3 z+2 q^{25 / 4} \sin 5 z-\cdots, \\
\vartheta_{2}(z, q) & =2 \sum_{n=0}^{\infty} q^{(n+1 / 2)^{2}} \cos (2 n+1) z \\
& =2 q^{1 / 4} \cos z+2 q^{9 / 4} \cos 3 z+2 q^{25 / 4} \cos 5 z+\cdots, \\
\vartheta_{3}(z, q) & =1+2 \sum_{n=1}^{\infty} q^{n^{2}} \cos 2 n z=1+2 q \cos 2 z+2 q^{4} \cos 4 z+2 q^{9} \cos 6 z+\cdots
\end{aligned}
$$

Работа выполнена при частичной поддержке Российского фонда фундаментальных исследований (грант № 97-01-00181).

(C) В.В. Зудилин 2000 


$$
\begin{aligned}
\vartheta_{4}(z, q) & =1+2 \sum_{n=1}^{\infty}(-1)^{n} q^{n^{2}} \cos 2 n z \\
& =1-2 q \cos 2 z+2 q^{4} \cos 4 z-2 q^{9} \cos 6 z+\cdots .
\end{aligned}
$$

Функции (0.1) являются цельми функциями аргумента $z$ при любом $|q|<1$. Параметр $q$ связан с модулярньм параметром $\tau$ формулой $q=e^{\pi i \tau}, \operatorname{Im} \tau>0$ (см. [6; $§ 21.1$ и $\S 21.7])$. Каждая из функций (0.1) удовлетворяет дифференциальному уравнению теплопроводности

$$
q \frac{\partial \vartheta(z, q)}{\partial q}=-\frac{1}{4} \frac{\partial^{2} \vartheta(z, q)}{\partial z^{2}}
$$

(см. $[6 ; \S 21.4])$. Кроме того, отношения тэта-функций (0.1) образуют замкнутую систему относительно дифференцирования по $z$ (см. [6; §21.6]).

В формулах, содержащих тэта-функции с одним и тем же параметром $q$, эта переменная традиционно опускается, как бы превращаясь из переменной в постоянную. С такой идеологией согласуется определение тәта-констант

$$
\vartheta_{2}=\vartheta_{2}(0), \quad \vartheta_{3}=\vartheta_{3}(0), \quad \vartheta_{4}=\vartheta_{4}(0)
$$

как значений четных тэта-функций (0.1) при $z=0$. Разумеется, функции (0.3) не являются постоянными и зависят от переменной $q$.

Еще в 1848 году Якоби [7] доказал, что каждая из тэта-констант (0.3) удовлетворяет одному и тому же дифференциальному уравнению третьего порядка. Уравнение Якоби в терминах $\delta$-дифференцирования принимает вид

$$
\begin{gathered}
\left(\vartheta^{2} \cdot \delta^{3} \vartheta-15 \vartheta \cdot \delta \vartheta \cdot \delta^{2} \vartheta+30(\delta \vartheta)^{3}\right)^{2}+32\left(\vartheta \cdot \delta^{2} \vartheta-3(\delta \vartheta)^{2}\right)^{3} \\
=4 \vartheta^{10}\left(\vartheta \cdot \delta^{2} \vartheta-3(\delta \vartheta)^{2}\right)^{3}, \quad \delta=q \frac{d}{d q}=\frac{1}{\pi i} \frac{d}{d \tau}
\end{gathered}
$$

(см., например, [8]). Веком позже К. Малер [9] доказал теорему об алгебраической независимости функций $\vartheta, \delta \vartheta, \delta^{2} \vartheta$, из которой, в частности, следовало, что ни одна из тэта-констант не удовлетворяет никакому алгебраическому дифференциальному уравнению второго порядка с коэффициентами из $\mathbb{C}[\tau]$ или из $\mathbb{C}[q]$. Мы сформулируем результат Малера из [9] в следующем виде.

ТЕОРема МАлеРА. Переменная $\tau$ и функции $q=e^{\pi i \tau}, \vartheta_{j}, \delta \vartheta_{j}, \delta^{2} \vartheta_{j} \partial \Omega_{я}$ любого $j=2,3,4$ алгебраччески независимы над полем $\mathbb{C}$.

Пусть $\mathscr{K}$ - дифференциальное замькание поля $\mathbb{C}(q)$, порожденное функциями (0.3) относительно $\delta$-дифференцирования. Из теоремы Малера следует, что степень трансцендентности поля $\mathscr{K}$ над $\mathbb{C}(q)$ равна 3 (см. также по этому поводу работы [10], [11]). Громоздкость уравнения (0.4) делает актуальньм вопрос о выборе образующих поля $\mathscr{K}$ - системы из трех алгебраически независимых над $\mathbb{C}(q)$ элементов $\mathscr{K}$ таких, что дифференциальные уравнения для них выглядят максимально просто.

Первый пример такой системы - логарифмические $\delta$-производные тэта-констант

$$
\psi_{2}=\frac{\delta \vartheta_{2}}{\vartheta_{2}}, \quad \psi_{3}=\frac{\delta \vartheta_{3}}{\vartheta_{3}}, \quad \psi_{4}=\frac{\delta \vartheta_{4}}{\vartheta_{4}}
$$

- привел в 1881 году М. Альфен [12]. Он доказал следующее утверждение. 
ТеОРема АЛЬфена. Функиии (0.5) удовлетворяют системе дифференциальных уравнений

$$
\delta\left(\psi_{2}+\psi_{3}\right)=4 \psi_{2} \psi_{3}, \quad \delta\left(\psi_{3}+\psi_{4}\right)=4 \psi_{3} \psi_{4}, \quad \delta\left(\psi_{4}+\psi_{2}\right)=4 \psi_{4} \psi_{2} .
$$

Менее компактная запись системы дифференциальных уравнений (0.6)

$$
\begin{aligned}
\delta \psi_{2} & =2\left(\psi_{2} \psi_{3}+\psi_{2} \psi_{4}-\psi_{3} \psi_{4}\right), \\
\delta \psi_{3} & =2\left(\psi_{2} \psi_{3}+\psi_{3} \psi_{4}-\psi_{2} \psi_{4}\right), \\
\delta \psi_{4} & =2\left(\psi_{2} \psi_{4}+\psi_{3} \psi_{4}-\psi_{2} \psi_{3}\right)
\end{aligned}
$$

демонстрирует замкнутость системы функций (0.5) относительно $\delta$-дифференцирования. Тэта-константы (0.3) связаны с функциями (0.5) хорошо известными соотношениями

$$
\psi_{3}-\psi_{4}=\frac{1}{4} \vartheta_{2}^{4}, \quad \psi_{2}-\psi_{4}=\frac{1}{4} \vartheta_{3}^{4}, \quad \psi_{2}-\psi_{3}=\frac{1}{4} \vartheta_{4}^{4},
$$

которые (в несколько иных обозначениях) можно найти, например, в $[13 ;$ гл. 11 , формулы (93.6)].

Другой пример "простой" системы образующих поля $\mathscr{K}$ был получен в 1916 году С. Рамануджаном [14].

Теорема РаманУДЖана. Функии

$$
\begin{gathered}
P(q)=1-24 \sum_{n=1}^{\infty} \sigma_{1}(n) q^{n} \\
Q(q)=1+240 \sum_{n=1}^{\infty} \sigma_{3}(n) q^{n}, \quad R(q)=1-504 \sum_{n=1}^{\infty} \sigma_{5}(n) q^{n}
\end{gathered}
$$

где $\sigma_{k}(n)=\sum_{d \mid n} d^{k}$, удовлетворяют системе дифференциальных уравнений

$$
\delta P=\frac{1}{12}\left(P^{2}-Q\right), \quad \delta Q=\frac{1}{3}(P Q-R), \quad \delta R=\frac{1}{2}\left(P R-Q^{2}\right) .
$$

Алгебраическая независимость функций (0.9) следует из упомянутого вьше результата Малера, а их алгебраичность над полем $\mathscr{K}$ получается из формул

$$
P\left(q^{2}\right)=1-24 \sum_{n=1}^{\infty} \frac{q^{2 n}}{\left(1-q^{2 n}\right)^{2}}=4\left(\frac{\delta \vartheta_{2}}{\vartheta_{2}}+\frac{\delta \vartheta_{3}}{\vartheta_{3}}+\frac{\delta \vartheta_{4}}{\vartheta_{4}}\right)
$$

(см., например, [6; §21.41]) и

$$
Q\left(q^{2}\right)=\frac{1}{2}\left(\vartheta_{2}^{8}+\vartheta_{3}^{8}+\vartheta_{4}^{8}\right), \quad R\left(q^{2}\right)=\frac{1}{2}\left(\vartheta_{4}^{4}-\vartheta_{2}^{4}\right)\left(\vartheta_{2}^{4}+\vartheta_{3}^{4}\right)\left(\vartheta_{3}^{4}+\vartheta_{4}^{4}\right)
$$

(см., например, [15; гл. III, теорема 1.16$]$ с небольшой опечаткой или $[16$; гл. 6 , упражнение 4] без опечаток); в правых частях соотношений (0.11) и (0.12) мы опустили параметр $q$, от которого зависят тэта-константы. Появление аргумента $q^{2}$ 
у функций Рамануджана в формулах (0.11) и (0.12) не является серьезным препятствием: согласно формулам

$\vartheta_{2}^{2}\left(q^{2}\right)=\frac{1}{2}\left(\vartheta_{3}^{2}(q)-\vartheta_{4}^{2}(q)\right), \quad \vartheta_{3}^{2}\left(q^{2}\right)=\frac{1}{2}\left(\vartheta_{3}^{2}(q)+\vartheta_{4}^{2}(q)\right), \quad \vartheta_{4}^{2}\left(q^{2}\right)=\vartheta_{3}(q) \vartheta_{4}(q)$

(см. [16; гл. 1, раздел 1.8]) дифференциальное замыкание поля $\mathbb{C}(q)$, порожденное функциями $\vartheta_{2}\left(q^{2}\right), \vartheta_{3}\left(q^{2}\right), \vartheta_{4}\left(q^{2}\right)$ относительно $\delta$-дифференцирования, с точностью до алгебраического расширения совпадает с $\mathscr{K}$.

Наш интерес к тэта-константам и их дифференциальньм свойствам инициирован следующим результатом Ю. В. Нестеренко [17], [18] в теории трансцендентных чисел.

Tеорема НестеренКо. Для любого $q_{0} \in \mathbb{C},\left|q_{0}\right|<1$, среди чисел

$$
q_{0}, P\left(q_{0}\right), Q\left(q_{0}\right), R\left(q_{0}\right)
$$

найдется по крайней мере три алгебраически независимых над полем $\mathbb{Q}$.

Разумеется, набор (0.13) можно заменить на $q_{0}, f_{1}\left(q_{0}\right), f_{2}\left(q_{0}\right), f_{3}\left(q_{0}\right)$ для любого выбора системы образующих $f_{1}, f_{2}, f_{3}$ поля $\mathscr{K}$ (но теорема была доказана именно для функций Рамануджана!). Одно из следствий теоремы Нестеренко - алгебраическая независимость над $\mathbb{Q}$ чисел $\pi, e^{\pi}, \Gamma(1 / 4)$.

Доказательство этой теоремы технически непросто и опирается на "хорошие" алгебраические свойства нелинейной системы диф ференциальных уравнений (0.10) и "хорошие" арифметические свойства разложений (0.9). Мы не будем конкретизировать последние обстоятельства, а только отметим, что подобными "хорошими" свойствами обладает также система (0.7) и разложения по степеням $q$ функций $(0.5)$ (см. [19]).

Сушествуют ли другие примеры подобных “хороших" систем функций и связывающих их нелинейных дифференциальных уравнений-вопрос открытый. В работе [20] указывается способ генерации подобных систем дифференциальных уравнений (типа системы Альфена) с помощью фуксовских линейных дифференциальных уравнений второго порядка, но их решения в отличие от функций (0.5), по всей видимости, не обладают "хорошими” арифметическими свойствами.

В настоящей работе мы получаем замкнутые системы дифференциальных уравнений в частных производных для многомерных тэта-констант и их логарифмических производных, исследуем алгебраические и модулярные свойства этих систем.

Зафиксируем размерность (род) $g$ и определим $g$-мерную тэта-функцию рядом

$$
\begin{gathered}
\vartheta(\boldsymbol{z}, \mathrm{T})=\sum_{\boldsymbol{n} \in \mathbb{Z}^{g}} \exp \left(\pi i^{t} \boldsymbol{n T} \boldsymbol{n}+2 i^{t} \boldsymbol{n z}\right) \\
\boldsymbol{z}=\left(\begin{array}{c}
z_{1} \\
\vdots \\
z_{g}
\end{array}\right) \in \mathbb{C}^{g}, \quad \mathrm{~T}=\left(\begin{array}{ccc}
\tau_{11} & \ldots & \tau_{1 g} \\
\vdots & \ddots & \vdots \\
\tau_{g 1} & \ldots & \tau_{g g}
\end{array}\right)
\end{gathered}
$$

где Т - симметричная комплексная матрица с положительно определенной мнимой частью (пространство $\mathfrak{H}_{g}$ размерности $g(g+1) / 2$ таких матриц является открытьм множеством в $\operatorname{Sym}_{g}(\mathbb{C})$ и называется верхним полупространством Зигеля). 
Векторы мы записываем в виде столбцов, обозначая индексом $t$ операцию транспонирования; таким образом, запись ${ }^{t} \boldsymbol{n z}$ обозначает скалярное произведение векторов $\boldsymbol{n}$ и $\boldsymbol{z}$.

Отметим, что ряд (0.14) сходится абсолютно и равномерно по $\boldsymbol{z}$ и Т на каждом компактном подмножестве в $\mathbb{C}^{g} \times \mathfrak{H}_{g}$, т.е. он определяет на этом множестве голоморфную функцию (см. [3; гл. II, предложение 1.1]).

$\mathrm{C}$ каждой матрищей $\mathrm{T} \in \mathfrak{H}_{g}$ можно связать решетку $\mathscr{L}_{\mathrm{T}}=\pi \mathrm{T} \mathbb{Z}^{g}+\pi \mathbb{Z}^{g} \subset \mathbb{C}^{g}$. Тэта-функция обладает свойством квазипериодичности относительно решетки $\mathscr{L}_{\mathrm{T}}$, а именно [3; гл. II, $\S 1]$

$$
\vartheta(\boldsymbol{z}+\pi \mathrm{T} \boldsymbol{m}+\pi \boldsymbol{n})=\exp \left(-\pi i^{t} \boldsymbol{m} \mathrm{T} \boldsymbol{m}-2 i^{t} \boldsymbol{m} \boldsymbol{z}\right) \vartheta(\boldsymbol{z}), \quad \boldsymbol{n}, \boldsymbol{m} \in \mathbb{Z}^{g} .
$$

Наряду с функцией (0.14) рассмотрим ее всевозможные сдвиги на полупериоды решетки $\mathscr{L}_{\mathrm{T}}-$ тәта-функиии с характеристиками -

$$
\begin{gathered}
\vartheta_{\boldsymbol{a}}(\boldsymbol{z}, \mathrm{T})=\sum_{\boldsymbol{n} \in \mathbb{Z}^{g}} \exp \left(\pi i^{t}\left(\boldsymbol{n}+\frac{1}{2} \boldsymbol{a}^{\prime}\right) \mathrm{T}\left(\boldsymbol{n}+\frac{1}{2} \boldsymbol{a}^{\prime}\right)+2 i^{t}\left(\boldsymbol{n}+\frac{1}{2} \boldsymbol{a}^{\prime}\right)\left(\boldsymbol{z}+\frac{\pi}{2} \boldsymbol{a}^{\prime \prime}\right)\right) \\
=\exp \left(\frac{\pi i t}{4} \boldsymbol{a}^{\prime} \mathrm{T} \boldsymbol{a}^{\prime}+i^{t} \boldsymbol{a}^{\prime}\left(\boldsymbol{z}+\frac{\pi}{2} \boldsymbol{a}^{\prime \prime}\right)\right) \vartheta\left(\boldsymbol{z}+\frac{\pi}{2} \mathrm{~T} \boldsymbol{a}^{\prime}+\frac{\pi}{2} \boldsymbol{a}^{\prime \prime}, \mathrm{T}\right) \\
\boldsymbol{a}=\left(\boldsymbol{a}^{\prime}, \boldsymbol{a}^{\prime \prime}\right) \in \mathbb{Z}^{2 g}
\end{gathered}
$$

которые мы также будем называть тэта-функциями. Свойство квазипериодичности функций (0.15) относительно решетки $\mathscr{L}_{\mathrm{T}}$ (см. [3; гл. II, §1]) позволяет рассматривать только функции с характеристиками $\boldsymbol{a} \in \mathbb{Z}^{2 g} / 2 \mathbb{Z}^{2 g}$. В частности, при $g=1$ мы получаем набор функций (0.1) (с отличающейся на постоянный множитель функцией $\left.\vartheta_{1}(z, q)\right)$. Сразу оговоримся, что множество $\mathfrak{K}=\mathbb{Z}^{2 g} / 2 \mathbb{Z}^{2 g}$, а также его факторизации и подмножества мы часто будем представлять себе как подмножество $\mathbb{Z}^{2 g}$ векторов, элементы которых нули и единицы.

Подобно одномерному случаю мы будем опускать параметр Т в записи тэта-функций и определим тәта-константы как значения четных тэта-функций (0.15) в точке $\boldsymbol{z}=\mathbf{0}$. Поскольку

$$
\vartheta_{\boldsymbol{a}}(-\boldsymbol{z})=(-1)^{|\boldsymbol{a}|} \vartheta_{\boldsymbol{a}}(\boldsymbol{z}), \quad|\boldsymbol{a}|={ }^{t} \boldsymbol{a}^{\prime} \boldsymbol{a}^{\prime \prime}(\bmod 2), \quad \boldsymbol{a}=\left(\boldsymbol{a}^{\prime}, \boldsymbol{a}^{\prime \prime}\right) \in \mathbb{Z}^{2 g}
$$

(см. $[1 ;$ ч. 2, гл. $7, \S 1])$, функция $\vartheta_{\boldsymbol{a}}(\boldsymbol{z})$ является четной тогда и только тогда, когда значение $|\boldsymbol{a}|$ четно, и нечетной в противном случае (см. [2; гл. V, $\S 1$, теорема 1]). Назовем характеристики четных тэта-функций четными и обозначим множество четных характеристик через $\mathfrak{K}^{*}$, а характеристики нечетных тэта-функций нечетнымми (и никак не обозначим отвечаюшее им множество). Как показывает несложный подсчет (см. [1, ч. 2, гл. $7, \S 3$, теорема IV]), количество четных характеристик равно $2^{g-1}\left(2^{g}+1\right)$.

Введем дифференциальные операторы

$$
\delta_{j j}=\frac{1}{\pi i} \frac{\partial}{\partial \tau_{j j}}, \quad j=1, \ldots, g, \quad \delta_{j k}=\frac{1}{2 \pi i} \frac{\partial}{\partial \tau_{j k}}=\delta_{k j}, \quad j, k=1, \ldots, g, j \neq k,
$$

и всевозможные частные “логарифмические” производные тэта-констант:

$$
\psi_{\boldsymbol{a}, j k}=\psi_{\boldsymbol{a}, j k}(\mathrm{~T})=\frac{\delta_{j k} \vartheta_{\boldsymbol{a}}}{\vartheta_{\boldsymbol{a}}}=\psi_{\boldsymbol{a}, k j}, \quad \boldsymbol{a} \in \mathfrak{K}^{*}, \quad j, k=1, \ldots, g .
$$


ТЕОРЕма 1. Функиии (0.16) удовлетворяют системе дифференииальных уравнений

$$
\begin{gathered}
\vartheta_{\boldsymbol{a}}^{4} \cdot \delta_{l s} \psi_{\boldsymbol{a}, j k}=-\vartheta_{\boldsymbol{a}}^{4} \cdot \psi_{\boldsymbol{a}, l s} \psi_{\boldsymbol{a}, j k}-\frac{1}{N_{j k l s}} \vartheta_{\boldsymbol{a}}^{4} \cdot \sum_{j_{1}, k_{1}, l_{1}, s_{1}}^{*} \psi_{\boldsymbol{a}, j_{1} k_{1}} \psi_{\boldsymbol{a}, l_{1} s_{1}} \\
+\frac{1}{N_{j k l s} \cdot 2^{g-2}} \sum_{\boldsymbol{b} \in \mathfrak{K}^{*}}(-1)^{|\boldsymbol{a}, \boldsymbol{b}|} \vartheta_{\boldsymbol{b}}^{4} \cdot \sum_{j_{1}, k_{1}, l_{1}, s_{1}}^{*} \psi_{\boldsymbol{b}, j_{1} k_{1}} \psi_{\boldsymbol{b}, l_{1} s_{1}} \\
\boldsymbol{a} \in \mathfrak{K}^{*}, \quad j, k, l, s=1, \ldots, g, \\
|\boldsymbol{a}, \boldsymbol{b}|={ }^{t} \boldsymbol{a}^{\prime} \boldsymbol{b}^{\prime \prime}-{ }^{t} \boldsymbol{b}^{\prime} \boldsymbol{a}^{\prime \prime}(\bmod 2), \quad \boldsymbol{a}=\left(\boldsymbol{a}^{\prime}, \boldsymbol{a}^{\prime \prime}\right) \in \mathbb{Z}^{2 g}, \quad \boldsymbol{b}=\left(\boldsymbol{b}^{\prime}, \boldsymbol{b}^{\prime \prime}\right) \in \mathbb{Z}^{2 g}
\end{gathered}
$$

где через $\sum^{*}$ обозначено суммирование по всем различным перестановкам множсества $\{j, k, l, s\}$, а через $N_{j k l s}$ - количество таких перестановок.

Дифференциальное замькание поля $\mathbb{C}(\mathrm{T})=\mathbb{C}\left(\tau_{j k}\right)_{j, k=1, \ldots, m}$, порожденное тэта-константами относительно $\delta_{j k}$-дифференцирований, $j, k=1, \ldots, g$, согласно теореме 1 имеет конечную степень алгебраической независимости над $\mathbb{C}(\mathrm{T})$. Точная оценка степени трансцендентности этого дифференциального поля была установлена в совместной работе Д. Бертрана и автора.

Tеорема [21]. Степень трансцендентности над $\mathbb{C}(\mathrm{T})$ дифференциального замыкания поля $\mathbb{C}(\mathrm{T})$, порожсденого тэта-константами относительно $\delta_{j k}$-дифференцирований, $j, k=1, \ldots, g$, равна $2 g^{2}+g$.

Система (0.17) может быть записана в более компактном виде, если ввести в рассмотрение квадратичные по $\boldsymbol{z} \in \mathbb{C}^{g}$ формы

$$
\begin{gathered}
\psi_{\boldsymbol{a}}=\psi_{\boldsymbol{a}}(\boldsymbol{z})={ }^{t} \boldsymbol{z} \Psi_{\boldsymbol{a}} \boldsymbol{z}, \quad \Psi_{\boldsymbol{a}}=\left(\psi_{\boldsymbol{a}, j k}\right)_{j, k=1, \ldots, g}, \quad \boldsymbol{a} \in \mathfrak{K}^{*} \\
\delta=\delta(\boldsymbol{z})={ }^{t} \boldsymbol{z} \Delta \boldsymbol{z}, \quad \Delta=\left(\delta_{j k}\right)_{j, k=1, \ldots, g} .
\end{gathered}
$$

Объект (0.19), разумеется, имеет смысл только при наличии следующего аргумента - мероморфной по $\mathrm{T} \in \mathfrak{H}_{g}$ функции или квадратичной формы по $\boldsymbol{z}$, коэффициенты которой - мероморфные по Т функции (нас будут интересовать только эти возможности). Естественным образом определяются операции умножения квадратичных форм на мероморфную по Т функцию и попарного (тензорного) перемножения двух квадратичных форм, результат которого - однородный многочлен четвертой степени (квартика) по $\boldsymbol{z}$. В этих обозначениях система $(0.17)$ записывается как равенство квартик:

$$
\vartheta_{\boldsymbol{a}}^{4} \cdot \delta \psi_{\boldsymbol{a}}=\frac{1}{2^{g-2}} \sum_{\boldsymbol{b} \in \mathfrak{K}^{*}}(-1)^{|\boldsymbol{a}, \boldsymbol{b}|} \vartheta_{\boldsymbol{b}}^{4} \cdot \psi_{\boldsymbol{b}}^{2}-2 \vartheta_{\boldsymbol{a}}^{4} \cdot \psi_{\boldsymbol{a}}^{2}, \quad \boldsymbol{a} \in \mathfrak{K}^{*}
$$

Несмотря на симметрию системы (0.20), она разве что отдаленно напоминает систему Альфена: во-первых, функции, входящие в систему (0.20), связаны большим количеством алгебраических соотношений и, во-вторых, множество особых точек этой системы содержит многообразие нулей всех тэта-констант (за информацией о которых мы отсылаем читателя к [2; гл. 5]).

Однако, как было показано в работах [22] и [23], в случае $g=2$ удается выписать систему, подобную (0.7). 
ТЕОРемА ОХИЯМЫ. Пусть четные характеристики $\boldsymbol{a}_{1}, \boldsymbol{a}_{2}, \boldsymbol{a}_{3}, \boldsymbol{a}_{4} \in \mathbb{Z}^{4} / 2 \mathbb{Z}^{4}$ образуют “вериины невырожденного параллелограмма" (т.е. $\boldsymbol{a}_{1}-\boldsymbol{a}_{2} \equiv \boldsymbol{a}_{3}-\boldsymbol{a}_{4}$ $\left.\left(\bmod 2 \mathbb{Z}^{4}\right)\right)$. Тогда отвечающие им функиии $\psi_{\boldsymbol{a}_{1}}, \psi_{\boldsymbol{a}_{2}}, \psi_{\boldsymbol{a}_{3}}, \psi_{\boldsymbol{a}_{4}}$ удовлетворяют дифференииальному уравнению

$\delta\left(\psi_{\boldsymbol{a}_{1}}+\psi_{\boldsymbol{a}_{2}}+\psi_{\boldsymbol{a}_{3}}+\psi_{\boldsymbol{a}_{4}}\right)=\left(\psi_{\boldsymbol{a}_{1}}+\psi_{\boldsymbol{a}_{2}}+\psi_{\boldsymbol{a}_{3}}+\psi_{\boldsymbol{a}_{4}}\right)^{2}-2\left(\psi_{\boldsymbol{a}_{1}}^{2}+\psi_{\boldsymbol{a}_{2}}^{2}+\psi_{\boldsymbol{a}_{3}}^{2}+\psi_{\boldsymbol{a}_{4}}^{2}\right)$.

К сожалению, в случае $g>2$ система дифференциальных уравнений для функций (0.18), подобная системе Альфена, до сих пор не получена. В случае $g=3$ из наших результатов (см. §6) получается система дифференциальных уравнений, в которой $\delta$-производные функций (0.18) выражаются как рациональные функции от них же, причем знаменатели имеют степень не выше 9.

Изложение устроено следующим образом. В $\S 1$ мы приводим два доказательства теоремы Альфена. Такое количество внимания, уделяемое этому результату, неслучайно: в следующих параграфах проводится аналогия между этими доказательствами и получением систем дифференциальных уравнений в случаях $g=2$ и $g=3$. В $\S 2$ и $\S 3$ мы указываем необходимые для дальнейшего свойства многомерных тэта-функций и в $\S 4$ доказываем теорему 1 . Случаю $g=2$ (и не только) мы уделяем внимание в $\S 5$ и $\S 6$, доказывая, в частности, теорему Охиямы. В $\S 6$ мы также обсуждаем случай $g=3$ и проблемы получения систем дифференциальных уравнений “без знаменателей” для функций (0.18), а в $\S 7$ приводим еще один пример системы дифференциальных уравнений, имеющей другую “структуру” по сравнению с системой (0.20). Модулярньм свойствам функций (0.16) и многомерньм обобщениям соотношений $(0.8)$ посвящен $\S 8$.

Автор благодарен профессору Ю.В. Нестеренко, инициировшему мою многомерную тэта-деятельность, за постоянное внимание и профессору Д. Бертрану, плодотворные беседы с которым способствовали написанию не только этой статьи, но и созданию совместной работы [21]. Настояшая работа была выполнена во время прохождения научной стажировки в Институте математики Университета П. и М. Кюри (Париж VI) и Центре Э. Борель (Институт А. Пуанкаре). Автор искренне признателен сотрудникам этих организаций за гостеприимство и теплую рабочую атмосферу. Особая благодарность фонду Островского за финансовую поддержку этой стажировки.

\section{§1. Два доказательства теоремы Альфена}

Уравнение теплопроводности (0.2), которому удовлетворяет любая из тэтафункций, содержит информацию о разложении функций (0.1) в степенной ряд по $z$.

ЛЕМма 1. В окрестности точки $z=0$ справедливы разложсения

$$
\vartheta_{j}(z)=\vartheta_{j} \cdot\left(1-2 \psi_{j} z^{2}+\frac{2}{3}\left(\psi_{j}^{2}+\delta \psi_{j}\right) z^{4}\right)+O\left(z^{6}\right), \quad j=2,3,4
$$


ДокАЗАТЕльство. Запишем первые члены тейлоровского разложения четных тэта-функций $(0.1)$ в точке $z=0$ :

$$
\vartheta_{j}(z)=\vartheta_{j} \cdot\left(1+a_{j} z^{2}+b_{j} z^{4}\right)+O\left(z^{6}\right), \quad j=2,3,4
$$

где $a_{j}=a_{j}(q)$ и $b_{j}=b_{j}(q)$ - некоторые функции переменной $q$, и воспользуемся уравнением теплопроводности $(0.2)$ :

$$
\begin{aligned}
-\frac{1}{4} \vartheta_{j}^{\prime \prime}(z) & =-\frac{1}{2} \vartheta_{j} \cdot\left(a_{j}+6 b_{j} z^{2}\right)+O\left(z^{4}\right), \\
\delta \vartheta_{j}(z) & =\delta \vartheta_{j}+\left(\delta \vartheta_{j} \cdot a_{j}+\vartheta_{j} \cdot \delta a_{j}\right) z^{2}+O\left(z^{4}\right),
\end{aligned} \quad j=2,3,4,
$$

откуда

$-\frac{1}{2} \vartheta_{j} \cdot\left(a_{j}+6 b_{j} z^{2}\right)+o\left(z^{2}\right)=\delta \vartheta_{j}+\left(\delta \vartheta_{j} \cdot a_{j}+\vartheta_{j} \cdot \delta a_{j}\right) z^{2}+O\left(z^{4}\right), \quad j=2,3,4$

После деления обеих частей на $\vartheta_{j}, j=2,3,4$, получаем

$$
-\frac{1}{2} a_{j}-3 b_{j} z^{2}+O\left(z^{4}\right)=\psi_{j}+\left(\psi_{j} a_{j}+\delta a_{j}\right) z^{2}+O\left(z^{4}\right), \quad j=2,3,4 .
$$

Сравнивая коэффициенты при одинаковых степенях $z$, находим:

$$
a_{j}=-2 \psi_{j}, \quad b_{j}=-\frac{1}{3}\left(\psi_{j} a_{j}+\delta a_{j}\right)=\frac{2}{3}\left(\psi_{j}^{2}+\delta \psi_{j}\right), \quad j=2,3,4,
$$

что и требовалось доказать.

ДОКАЗАТЕЛЬСТВо ТЕОРЕМЫ АЛЬФЕНА. Воспользуемся только формулами сложения

$$
\begin{aligned}
& \vartheta_{2}(z+y) \vartheta_{2}(z-y) \vartheta_{2}^{2}=\vartheta_{3}^{2}(z) \vartheta_{3}^{2}(y)-\vartheta_{4}^{2}(z) \vartheta_{4}^{2}(y), \\
& \vartheta_{3}(z+y) \vartheta_{3}(z-y) \vartheta_{3}^{2}=\vartheta_{2}^{2}(z) \vartheta_{2}^{2}(y)+\vartheta_{4}^{2}(z) \vartheta_{4}^{2}(y), \\
& \vartheta_{4}(z+y) \vartheta_{4}(z-y) \vartheta_{4}^{2}=\vartheta_{3}^{2}(z) \vartheta_{3}^{2}(y)-\vartheta_{2}^{2}(z) \vartheta_{2}^{2}(y)
\end{aligned}
$$

(см. [3; гл. I, $\S 5$, таблица 3$]$ ), точнее формулами удвоения

$\vartheta_{2}(2 z) \vartheta_{2}^{3}=\vartheta_{3}^{4}(z)-\vartheta_{4}^{4}(z), \quad \vartheta_{3}(2 z) \vartheta_{3}^{3}=\vartheta_{2}^{4}(z)+\vartheta_{4}^{4}(z), \quad \vartheta_{4}(2 z) \vartheta_{4}^{3}=\vartheta_{3}^{4}(z)-\vartheta_{2}^{4}(z)$, получающимися из формул (1.2) после подстановки $y=z$.

Учитьвая разложения

$$
\begin{aligned}
\vartheta_{j}(2 z) & =\vartheta_{j} \cdot\left(1+4 a_{j} z^{2}+16 b_{j} z^{4}\right)+o\left(z^{4}\right), \\
\vartheta_{j}^{4}(z) & =\vartheta_{j}^{4} \cdot\left(1+4 a_{j} z^{2}+\left(6 a_{j}^{2}+4 b_{j}\right) z^{4}\right)+o\left(z^{4}\right), \quad j=2,3,4
\end{aligned}
$$

(коэффициенты $a_{j}, b_{j}$ определяются в соответствии с $(1.1)$ ), приравняем коэффициенты при $z^{0}, z^{2}, z^{4}$ в форомулах удвоения (1.3):

$$
\begin{gathered}
\vartheta_{2}^{4}-\vartheta_{3}^{4}+\vartheta_{4}^{4}=0, \quad \vartheta_{2}^{4} a_{2}-\vartheta_{3}^{4} a_{3}+\vartheta_{4}^{4} a_{4}=0, \\
16 \vartheta_{2}^{4} b_{2}=\vartheta_{3}^{4}\left(6 a_{3}^{2}+4 b_{3}\right)-\vartheta_{4}^{4}\left(6 a_{4}^{2}+4 b_{4}\right), \\
16 \vartheta_{3}^{4} b_{3}=\vartheta_{2}^{4}\left(6 a_{2}^{2}+4 b_{2}\right)+\vartheta_{4}^{4}\left(6 a_{4}^{2}+4 b_{4}\right), \\
16 \vartheta_{4}^{4} b_{4}=\vartheta_{3}^{4}\left(6 a_{3}^{2}+4 b_{3}\right)-\vartheta_{2}^{4}\left(6 a_{2}^{2}+4 b_{2}\right) .
\end{gathered}
$$


Решая линейную систему уравнений (1.5) относительно $\vartheta_{j}^{4} b_{j}, j=2,3,4$, получаем

$$
\begin{aligned}
& \vartheta_{2}^{4} b_{2}=\frac{1}{6}\left(\vartheta_{2}^{4} a_{2}^{2}+2 \vartheta_{3}^{4} a_{3}^{2}-2 \vartheta_{4}^{4} a_{4}^{2}\right), \\
& \vartheta_{3}^{4} b_{3}=\frac{1}{6}\left(2 \vartheta_{2}^{4} a_{2}^{2}+\vartheta_{3}^{4} a_{3}^{2}+2 \vartheta_{4}^{4} a_{4}^{2}\right), \\
& \vartheta_{4}^{4} b_{4}=\frac{1}{6}\left(-2 \vartheta_{2}^{4} a_{2}^{2}+2 \vartheta_{3}^{4} a_{3}^{2}+\vartheta_{4}^{4} a_{4}^{2}\right) .
\end{aligned}
$$

Подставляя соотношения (1.1), найденные в лемме 1, в (1.6), получаем систему дифференциальных уравнений, связывающих функции (0.5):

$$
\begin{aligned}
& \frac{2}{3} \vartheta_{2}^{4}\left(\psi_{2}^{2}+\delta \psi_{2}\right)=\frac{2}{3}\left(\vartheta_{2}^{4} \psi_{2}^{2}+2 \vartheta_{3}^{4} \psi_{3}^{2}-2 \vartheta_{4}^{4} \psi_{4}^{2}\right), \\
& \frac{2}{3} \vartheta_{3}^{4}\left(\psi_{3}^{2}+\delta \psi_{3}\right)=\frac{2}{3}\left(2 \vartheta_{2}^{4} \psi_{2}^{2}+\vartheta_{3}^{4} \psi_{3}^{2}+2 \vartheta_{4}^{4} \psi_{4}^{2}\right), \\
& \frac{2}{3} \vartheta_{4}^{4}\left(\psi_{4}^{2}+\delta \psi_{4}\right)=\frac{2}{3}\left(-2 \vartheta_{2}^{4} \psi_{2}^{2}+2 \vartheta_{3}^{4} \psi_{3}^{2}+\vartheta_{4}^{4} \psi_{4}^{2}\right)
\end{aligned}
$$

откуда

$\delta \psi_{2}=2\left(\frac{\vartheta_{3}^{4}}{\vartheta_{2}^{4}} \psi_{3}^{2}-\frac{\vartheta_{4}^{4}}{\vartheta_{2}^{4}} \psi_{4}^{2}\right), \quad \delta \psi_{3}=2\left(\frac{\vartheta_{2}^{4}}{\vartheta_{3}^{4}} \psi_{2}^{2}+\frac{\vartheta_{4}^{4}}{\vartheta_{3}^{4}} \psi_{4}^{2}\right), \quad \delta \psi_{4}=2\left(\frac{\vartheta_{3}^{4}}{\vartheta_{4}^{4}} \psi_{3}^{2}-\frac{\vartheta_{2}^{4}}{\vartheta_{4}^{4}} \psi_{2}^{2}\right)$

Перепишем теперь соотношения (1.4) в виде

$$
\vartheta_{2}^{4}-\vartheta_{3}^{4}+\vartheta_{4}^{4}=0, \quad \vartheta_{2}^{4} \psi_{2}-\vartheta_{3}^{4} \psi_{3}+\vartheta_{4}^{4} \psi_{4}=0
$$

Из них, в частности, следуют выражения для отношений четвертых степеней тэтафункций:

$$
\frac{\vartheta_{3}^{4}}{\vartheta_{2}^{4}}=\frac{\psi_{2}-\psi_{4}}{\psi_{3}-\psi_{4}}, \quad \frac{\vartheta_{4}^{4}}{\vartheta_{2}^{4}}=\frac{\psi_{2}-\psi_{3}}{\psi_{3}-\psi_{4}}
$$

что позволяет легко получить из дифференциальных уравнений (1.7) систему (0.7). Так, для первого уравнения системы (1.7) имеем

$$
\begin{aligned}
\delta \psi_{2} & =2\left(\frac{\psi_{2}-\psi_{4}}{\psi_{3}-\psi_{4}} \psi_{3}^{2}-\frac{\psi_{2}-\psi_{3}}{\psi_{3}-\psi_{4}} \psi_{4}^{2}\right)=2 \frac{\psi_{2}\left(\psi_{3}^{2}-\psi_{4}^{2}\right)-\psi_{3} \psi_{4}\left(\psi_{3}-\psi_{4}\right)}{\psi_{3}-\psi_{4}} \\
& =2\left(\psi_{2} \psi_{3}+\psi_{2} \psi_{4}-\psi_{3} \psi_{4}\right)
\end{aligned}
$$

и аналогично поступаем со вторым и третьим уравнениями системы (1.7). Для завершения доказательства теоремы в очередной раз отметим эквивалентность систем (0.7) и (0.6).

Докажем тождества (0.8). Для этого перепишем соотношения (1.8) в виде

$$
\frac{\psi_{3}-\psi_{4}}{\vartheta_{2}^{4}}=\frac{\psi_{2}-\psi_{4}}{\vartheta_{3}^{4}}=\frac{\psi_{2}-\psi_{3}}{\vartheta_{4}^{4}}=\varkappa(q)
$$


и покажем с помощью полученной системы дифференциальных уравнений (0.7) и соотношений

$$
\delta\left(\vartheta_{j}^{-4}\right)=-4 \vartheta_{j}^{-5} \cdot \delta \vartheta_{j}=-4 \vartheta_{j}^{-4} \psi_{j}, \quad j=2,3,4,
$$

что $\varkappa(q)=$ const. Последнее утверждение следует из того, что

$$
\begin{aligned}
\delta \varkappa & =\delta\left(\vartheta_{2}^{-4}\left(\psi_{3}-\psi_{4}\right)\right)=-\vartheta_{2}^{-4}\left(4 \psi_{2}\left(\psi_{3}-\psi_{4}\right)+\delta \psi_{3}-\delta \psi_{4}\right) \\
& =-2 \vartheta_{2}^{-4}\left(2 \psi_{2}\left(\psi_{3}-\psi_{4}\right)+\left(\psi_{2} \psi_{3}+\psi_{3} \psi_{4}-\psi_{2} \psi_{4}\right)-\left(\psi_{2} \psi_{4}+\psi_{3} \psi_{4}-\psi_{2} \psi_{3}\right)\right)=0 .
\end{aligned}
$$

Заметим теперь, что $\vartheta_{3}=1+2 q+O\left(q^{2}\right), \vartheta_{4}=1-2 q+O\left(q^{2}\right)$, откуда $\psi_{3}=2 q+O\left(q^{2}\right)$, $\psi_{4}=-2 q+O\left(q^{2}\right)$, и $\vartheta_{2}^{4}=16 q+O\left(q^{2}\right)$; поэтому

$$
\varkappa=\frac{\psi_{3}-\psi_{4}}{\vartheta_{2}^{4}}=\frac{1}{4}+O(q)
$$

т.е. $\varkappa=1 / 4$. Это доказывает тождества $(0.8)$.

ОЧЕНЬ ПРОСТОЕ ДОКАЗАТЕЛЬСТВО ТЕОРЕМЫ АЛЬФЕНА. ИЗ ОбШеЙ форМУЛЫ сложения для тэта-функций следуют соотношения

$$
\vartheta_{j}^{2}(z) \vartheta_{k}^{2}(z)=\frac{1}{2}\left(\vartheta_{j}(2 z) \vartheta_{j}(0) \vartheta_{k}^{2}(0)+\vartheta_{j}^{2}(0) \vartheta_{k}(2 z) \vartheta_{k}(0)\right), \quad j, k=2,3,4, \quad j \neq k
$$

(см. [6; упражнения 1, 2 к гл. 21]).

Согласно лемме 1

$$
\begin{aligned}
& \vartheta_{j}^{2}(z) \vartheta_{k}^{2}(z)=\vartheta_{j}^{2} \vartheta_{k}^{2} \cdot\left(1-4\left(\psi_{j}+\psi_{k}\right) z^{2}\right. \\
& \left.+16 \psi_{j} \psi_{k} z^{4}+\frac{16}{3}\left(\psi_{j}^{2}+\psi_{k}^{2}\right) z^{4}+\frac{4}{3} \delta\left(\psi_{j}+\psi_{k}\right) z^{4}\right)+O\left(z^{6}\right), \\
& \frac{\vartheta_{j}(2 z) \vartheta_{j} \vartheta_{k}^{2}+\vartheta_{j}^{2} \vartheta_{k}(2 z) \vartheta_{k}}{2}=\vartheta_{j}^{2} \vartheta_{k}^{2} \cdot\left(1-4\left(\psi_{j}+\psi_{k}\right) z^{2}\right. \\
& \left.+\frac{16}{3}\left(\psi_{j}^{2}+\psi_{k}^{2}\right) z^{4}+\frac{16}{3} \delta\left(\psi_{j}+\psi_{k}\right) z^{4}\right)+O\left(z^{6}\right)
\end{aligned}
$$

Подставляя разложения (1.11) в тождества (1.10) и сравнивая коэффициенты при $z^{4}$, получаем систему дифференциальных уравнений (0.6).

ЗАмечАниЕ. Разумеется, сам Альфен [12] приводит простое доказательство своей теоремы. Основным достоинством “длинного” доказательства являются полученные с его помошью тождества (0.8).

\section{§2. Разложения тэта-функций}

Пусть размерность $g$ фиксирована, $g$-мерные тэта-функции (с характеристиками) определяются формулами (0.15), через $\mathfrak{K}^{*}$ обозначено множество четных характеристик. В этом параграфе мы докажем следующее многомерное обобшение леммы 1.

ЛЕмма 2. В окрестности точки $\boldsymbol{z}=\mathbf{0}$ справедливы разложсения

$$
\vartheta_{\boldsymbol{a}}(\boldsymbol{z})=\vartheta_{\boldsymbol{a}} \cdot\left(1-2 \psi_{\boldsymbol{a}}+\frac{2}{3} \psi_{\boldsymbol{a}}^{2}+\frac{2}{3} \delta \psi_{\boldsymbol{a}}\right)+O\left(\boldsymbol{z}^{6}\right), \quad \boldsymbol{a} \in \mathfrak{K}^{*},
$$


где квадратичные формы $\psi_{\boldsymbol{a}}, \boldsymbol{a} \in \mathfrak{K}^{*}$, и $\delta$ определяются формулами (0.18) и (0.19). Кроме того, выполнены соотночения

$$
\begin{aligned}
\delta_{j k} \psi_{\boldsymbol{a}, l m}+\psi_{\boldsymbol{a}, j k} \psi_{\boldsymbol{a}, l m} & =\delta_{j l} \psi_{\boldsymbol{a}, k m}+\psi_{\boldsymbol{a}, j l} \psi_{\boldsymbol{a}, k m}, \quad \delta_{j k} \psi_{\boldsymbol{a}, l m}=\delta_{l m} \psi_{\boldsymbol{a}, j k}, \\
\boldsymbol{a} & \in \mathfrak{K}^{*}, \quad j, k, l, m=1, \ldots, g .
\end{aligned}
$$

Символом $O\left(z^{n}\right), n=0,1,2, \ldots$, мы обозначаем класс голоморфных в точке $\boldsymbol{z}=\mathbf{0}$ функций $f(\boldsymbol{z})$, разложение в степенной ряд которых начинается со степени $n$, т.e.

$$
\frac{\partial^{m_{1}+\cdots+m_{g} f}}{\partial z_{1}^{m_{1}} \cdots \partial z_{g}^{m_{g}}}(\mathbf{0})=0, \quad m_{1}+\cdots+m_{g}<n .
$$

Несложно проверить (мы пользуемся стандартной записью О-символики), что $O\left(\boldsymbol{z}^{n}\right)+O\left(\boldsymbol{z}^{k}\right)=O\left(\boldsymbol{z}^{\min \{n, k\}}\right)$ и $O\left(\boldsymbol{z}^{n}\right) \cdot O\left(\boldsymbol{z}^{k}\right)=O\left(\boldsymbol{z}^{n+k}\right)$.

Как и в одномерном случае, для доказательства леммы 2 нам понадобится многомерный аналог уравнения теплопроводности для функций (0.15).

Лемма 3 [1, ч. 1, гл. 1, §5, теорема XIII]. Любая из функиий (0.15) удовлетворяет дифференциальным уравнениям

$$
\delta_{j k} \vartheta_{\boldsymbol{a}}(\boldsymbol{z}, \mathrm{T})=-\frac{1}{4} \frac{\partial^{2}}{\partial z_{j} \partial z_{k}} \vartheta_{\boldsymbol{a}}(\boldsymbol{z}, \mathrm{T}), \quad j, k=1, \ldots, g, \quad \boldsymbol{a} \in \mathbb{Z}^{2 g}
$$

Это утверждение доказьвается непосредственной проверкой для рядов (0.15).

Следующее техническое утверждение не имеет прямого отношения к тэта-функциям.

ЛЕМма 4. Пусть $V(\boldsymbol{z})$ - однородный многочлен четвертой степени от переменных $z_{1}, \ldots, z_{g} ; \Phi=\left(\varphi_{j k}\right)_{j, k=1, \ldots, g} u \Psi=\left(\psi_{j k}\right)_{j, k=1, \ldots, g}-$ cимметричные квадратные матрицы. Известно, что

$$
\frac{\partial^{2} V}{\partial z_{j} \partial z_{k}}=\varphi_{j k} \cdot{ }^{t} \boldsymbol{z} \boldsymbol{z}, \quad j, k=1, \ldots, g
$$

Тогда

$$
V(\boldsymbol{z})=\frac{1}{12}\left({ }^{t} \boldsymbol{z} \Phi \boldsymbol{z}\right)\left({ }^{t} \boldsymbol{z} \Psi \boldsymbol{z}\right)
$$

и выполнень равенства

$$
\begin{gathered}
\varphi_{j k} \psi_{l m}=\varphi_{j l} \psi_{k m}=\varphi_{j m} \psi_{k l}=\varphi_{k l} \psi_{j m}=\varphi_{k m} \psi_{j l}=\varphi_{l m} \psi_{j k} \\
j, k, l, m=1, \ldots, g .
\end{gathered}
$$


ДокАЗАТЕЛЬСТво. Представим многочлен $V(\boldsymbol{z})$ в “симметричной” форме

$$
V(\boldsymbol{z})=\sum_{l_{1}, l_{2}, l_{3}, l_{4}=1}^{g} v_{l_{1} l_{2} l_{3} l_{4}} z_{l_{1}} z_{l_{2}} z_{l_{3}} z_{l_{4}}
$$

где $v_{l_{1} l_{2} l_{3} l_{4}}=v_{\sigma\left(l_{1} l_{2} l_{3} l_{4}\right)}$ для любой перестановки $\sigma$ индексов $l_{1}, l_{2}, l_{3}, l_{4}$. Тогда

$$
\begin{aligned}
\frac{\partial^{2} V}{\partial z_{j} \partial z_{k}} & =\frac{\partial^{2}}{\partial z_{j} \partial z_{k}} \sum_{l_{1}, l_{2}, l_{3}, l_{4}=1}^{g} v_{l_{1} l_{2} l_{3} l_{4}} z_{l_{1}} z_{l_{2}} z_{l_{3}} z_{l_{4}} \\
& =\sum_{l_{3}, l_{4}=1}^{g} v_{j k l_{3} l_{4}} z_{l_{3}} z_{l_{4}}+\sum_{l_{2}, l_{4}=1}^{g} v_{j l_{2} k l_{4}} z_{l_{2}} z_{l_{4}}+\cdots+\sum_{l_{1}, l_{2}=1}^{g} v_{l_{1} l_{2} j k} z_{l_{1}} z_{l_{2}} \\
& =\sum_{l_{3}, l_{4}=1}^{g}\left(v_{j k l_{3} l_{4}}+v_{j l_{3} k l_{4}}+\cdots+v_{l_{3} l_{4} j k}\right) z_{l_{3}} z_{l_{4}}=12 \sum_{l_{3}, l_{4}=1}^{g} v_{j k l_{3} l_{4}} z_{l_{3}} z_{l_{4}} .
\end{aligned}
$$

Сравнивая коэффициенты получающихся квадратичных форм с условиями (2.3), находим:

$$
v_{j k l_{3} l_{4}}=\frac{1}{12} \varphi_{j k} \psi_{l_{3} l_{4}}, \quad j, k, l_{3}, l_{4}=1, \ldots, g
$$

Следовательно,

$$
\begin{aligned}
V(\boldsymbol{z}) & =\frac{1}{12} \sum_{l_{1}, l_{2}, l_{3}, l_{4}=1}^{g} \varphi_{l_{1} l_{2}} \psi_{l_{3} l_{4}} z_{l_{1}} z_{l_{2}} z_{l_{3}} z_{l_{4}} \\
& =\frac{1}{12}\left(\sum_{l_{1}, l_{2}=1}^{g} \varphi_{l_{1} l_{2}} z_{l_{1}} z_{l_{2}}\right)\left(\sum_{l_{3}, l_{4}=1}^{g} \psi_{l_{3} l_{4}} z_{l_{3}} z_{l_{4}}\right)=\frac{1}{12}\left({ }^{t} \boldsymbol{z} \boldsymbol{z}\right)\left({ }^{t} \boldsymbol{z} \Psi \boldsymbol{z}\right),
\end{aligned}
$$

что доказывает соотношение (2.4). Равенства (2.5) следуют из (2.7) и симметрии коэффициентов формы (2.6).

ДоКАЗАТЕЛЬСТВо ЛЕмМы 2 не зависит от выбора четной характеристики $\boldsymbol{a}$, поэтому мы исключим ее из нижнего индекса.

Разложение четной функции в степенной ряд имеет вид

$$
\vartheta(\boldsymbol{z})=\vartheta \cdot(1+U(\boldsymbol{z})+V(\boldsymbol{z}))+O\left(\boldsymbol{z}^{6}\right), \quad \vartheta=\vartheta(\mathbf{0})
$$

где $U(\boldsymbol{z})$ - квадратичная форма (однородный многочлен степени 2), $V(\boldsymbol{z})$ - квартика (однородньй многочлен степени 4), коэффициенты этих форм являются мероморфными по $\mathrm{T} \in \mathfrak{H}_{g}$ функциями.

Воспользуемся для разложения (2.8) леммой 3. Имеем

$$
\begin{aligned}
\delta_{j k} \vartheta(\boldsymbol{z}) & =\delta_{j k} \vartheta+\delta_{j k} \vartheta \cdot U(\boldsymbol{z})+\vartheta \cdot \delta_{j k} U(\boldsymbol{z})+O\left(\boldsymbol{z}^{4}\right), \\
\frac{\partial^{2}}{\partial z_{j} \partial z_{k}} \vartheta(\boldsymbol{z}) & =\vartheta \cdot\left(\frac{\partial^{2} U(\boldsymbol{z})}{\partial z_{j} \partial z_{k}}+\frac{\partial^{2} V(\boldsymbol{z})}{\partial z_{j} \partial z_{k}}\right)+O\left(\boldsymbol{z}^{4}\right), \quad j, k=1, \ldots, g, \quad
\end{aligned}
$$


откуда (мы делим обе части уравнений (2.2) на $\vartheta$ )

$$
\begin{gathered}
\psi_{j k}+\psi_{j k} U(\boldsymbol{z})+\delta_{j k} U(\boldsymbol{z})+O\left(\boldsymbol{z}^{4}\right)=-\frac{1}{4}\left(\frac{\partial^{2} U(\boldsymbol{z})}{\partial z_{j} \partial z_{k}}+\frac{\partial^{2} V(\boldsymbol{z})}{\partial z_{j} \partial z_{k}}\right)+O\left(\boldsymbol{z}^{4}\right), \\
j, k=1, \ldots, g .
\end{gathered}
$$

Сравнение однородных компонент в разложениях (2.9) дает соотношения

$$
\begin{gathered}
\frac{\partial^{2} U(\boldsymbol{z})}{\partial z_{j} \partial z_{k}}=-4 \psi_{j k}, \quad j, k=1, \ldots, g \\
\frac{\partial^{2} V(\boldsymbol{z})}{\partial z_{j} \partial z_{k}}=-4\left(\psi_{j k} U(\boldsymbol{z})+\delta_{j k} U(\boldsymbol{z})\right), \quad j, k=1, \ldots, g .
\end{gathered}
$$

Соотношения (2.10) означают, что

$$
U(\boldsymbol{z})=-2^{t} \boldsymbol{z} \Psi \boldsymbol{z}=-2 \psi
$$

Подстановка (2.12) в (2.11) приводит к формулам

$$
\frac{\partial^{2} V(\boldsymbol{z})}{\partial z_{j} \partial z_{k}}=8\left(\psi_{j k}+\delta_{j k}\right)^{t} \boldsymbol{z} \boldsymbol{z}, \quad j, k=1, \ldots, g
$$

откуда согласно лемме 4

$$
V(\boldsymbol{z})=\frac{2}{3}\left({ }^{t} \boldsymbol{z} \Psi \boldsymbol{z}\right)^{2}+\frac{2}{3}\left({ }^{t} \boldsymbol{z} \Delta \boldsymbol{z}\right)\left({ }^{t} \boldsymbol{z} \Psi \boldsymbol{z}\right)=\frac{2}{3} \psi^{2}+\frac{2}{3} \delta \psi
$$

и

$$
\left(\psi_{j k}+\delta_{j k}\right) \psi_{l m}=\left(\psi_{j l}+\delta_{j l}\right) \psi_{k m}=\cdots=\left(\psi_{l m}+\delta_{l m}\right) \psi_{j k}, \quad j, k, l, m=1, \ldots, g
$$

Лемма 2 доказана.

\section{§3. Соотношения Римана}

Именно эта формула используется в большинстве приложений.

Д. Мамфорд

Цитата из книги [3], вьнесенная в эпиграфф этого параграфа, относится к coomношениям Римана [3; гл. II, §6], которые для тэта-функций (0.15) записываются в виде

$$
\begin{gathered}
(-1)^{t}\left(\boldsymbol{c}^{\prime}+\boldsymbol{d}^{\prime}\right) \boldsymbol{a}^{\prime \prime} \vartheta_{\boldsymbol{a}+\boldsymbol{c}+\boldsymbol{d}}\left(\frac{\boldsymbol{z}_{1}+\boldsymbol{z}_{2}+\boldsymbol{z}_{3}+\boldsymbol{z}_{4}}{2}\right) \vartheta_{\boldsymbol{a}+\boldsymbol{c}}\left(\frac{\boldsymbol{z}_{1}+\boldsymbol{z}_{2}-\boldsymbol{z}_{3}-\boldsymbol{z}_{4}}{2}\right) \\
\times \vartheta_{\boldsymbol{a}+\boldsymbol{d}}\left(\frac{\boldsymbol{z}_{1}-\boldsymbol{z}_{2}+\boldsymbol{z}_{3}-\boldsymbol{z}_{4}}{2}\right) \vartheta_{\boldsymbol{a}}\left(\frac{\boldsymbol{z}_{1}-\boldsymbol{z}_{2}-\boldsymbol{z}_{3}+\boldsymbol{z}_{4}}{2}\right) \\
=\frac{1}{2^{g}} \sum_{\boldsymbol{b} \in \mathfrak{K}}(-1)^{|\boldsymbol{a}, \boldsymbol{b}|}(-1)^{t\left(\boldsymbol{c}^{\prime}+\boldsymbol{d}^{\prime}\right) \boldsymbol{b}^{\prime \prime}} \vartheta_{\boldsymbol{b}+\boldsymbol{c}+\boldsymbol{d}}\left(\boldsymbol{z}_{1}\right) \vartheta_{\boldsymbol{b}+\boldsymbol{c}}\left(\boldsymbol{z}_{2}\right) \vartheta_{\boldsymbol{b}+\boldsymbol{d}}\left(\boldsymbol{z}_{3}\right) \vartheta_{\boldsymbol{b}}\left(\boldsymbol{z}_{4}\right), \\
\boldsymbol{a}, \boldsymbol{c}, \boldsymbol{d} \in \mathfrak{K}=\mathbb{Z}^{2 g} / 2 \mathbb{Z}^{2 g}
\end{gathered}
$$


(см. [1; ч. 2, гл. VII, $\S 10$, теорема XXXVIII]). На самом деле в дальнейшем основное наше внимание будет приковано к частному случаю соотношений Римана, а именно

$$
\begin{gathered}
(-1)^{t}\left(\boldsymbol{c}^{\prime}+\boldsymbol{d}^{\prime}\right) \boldsymbol{a}^{\prime \prime} \vartheta_{\boldsymbol{a}+\boldsymbol{c}+\boldsymbol{d}}(\boldsymbol{z}) \vartheta_{\boldsymbol{a}+\boldsymbol{c}}(\boldsymbol{z}) \vartheta_{\boldsymbol{a}+\boldsymbol{d}}(\boldsymbol{z}) \vartheta_{\boldsymbol{a}}(\boldsymbol{z}) \\
=\frac{1}{2^{g}} \sum_{\boldsymbol{b} \in \mathfrak{K}}(-1)^{|\boldsymbol{a}, \boldsymbol{b}|}(-1)^{t}\left(\boldsymbol{c}^{\prime}+\boldsymbol{d}^{\prime}\right) \boldsymbol{b}^{\prime \prime} \vartheta_{\boldsymbol{b}+\boldsymbol{c}+\boldsymbol{d}}(2 \boldsymbol{z}) \vartheta_{\boldsymbol{b}+\boldsymbol{c}}(\mathbf{0}) \vartheta_{\boldsymbol{b}+\boldsymbol{d}}(\mathbf{0}) \vartheta_{\boldsymbol{b}}(\mathbf{0}), \\
\boldsymbol{a}, \boldsymbol{c}, \boldsymbol{d} \in \mathfrak{K},
\end{gathered}
$$

которьй получается из формул (3.1) подстановкой $\boldsymbol{z}_{1}=2 \boldsymbol{z}, \boldsymbol{z}_{2}=\boldsymbol{z}_{3}=\boldsymbol{z}_{4}=\mathbf{0}$.

Лемма 5. Для любой четной характеристики а справедливо тождество

$$
\vartheta_{\boldsymbol{a}}^{4}(\boldsymbol{z})=\frac{1}{2^{g}} \sum_{\boldsymbol{b} \in \mathfrak{K}^{*}}(-1)^{|\boldsymbol{a}, \boldsymbol{b}|} \vartheta_{\boldsymbol{b}}(2 \boldsymbol{z}) \vartheta_{\boldsymbol{b}}^{3}(\mathbf{0}) .
$$

ДокАЗАТЕЛьство. Для доказательства достаточно положить $\boldsymbol{c}=\boldsymbol{d}=\mathbf{0}$ в тождестве (3.2) и заметить, что все слагаемые в правой части, отвечающие нечетным характеристикам, пропадают.

Для квадратной симметричной матрицы размера $2^{g-1}\left(2^{g}+1\right)$, отвечающей соотношениям (3.3), введем обозначение

$$
\mathrm{M}=\frac{1}{2^{g}}\left((-1)^{|\boldsymbol{a}, \boldsymbol{b}|}\right)_{\boldsymbol{a}, \boldsymbol{b} \in \mathfrak{K}^{*}} \cdot
$$

При изучении свойств этой матрицы нам понадобятся два простых вспомогательных утверждения (из теории суммирования характеров).

ЛЕмма 6. Справедливь тождества

$$
\sum_{\boldsymbol{b} \in \mathfrak{K}}(-1)^{|\boldsymbol{a}, \boldsymbol{b}|}=\left\{\begin{array}{ll}
2^{2 g}, & \text { если } \boldsymbol{a}=\mathbf{0}, \\
0 & \text { в противном случае }
\end{array} \quad \boldsymbol{a} \in \mathfrak{K} .\right.
$$

ДокАЗАТЕльство. Случай $\boldsymbol{a}=\mathbf{0}$ тривиален: в сумме участвует $2^{2 g}$ слагаемых, каждое из которых равно 1 . В случае $\boldsymbol{a} \neq \mathbf{0}$ выберем характеристику $\boldsymbol{c}$ так, чтобы величина $|\boldsymbol{a}, \boldsymbol{c}|$ была нечетной (для этого достаточно выбрать характеристику с одной единственной единицей на $j$-м месте, где $\left.\boldsymbol{a}_{j \pm g} \neq 0\right)$, и умножим сумму в левой части $(3.4)$ на $(-1)^{|\boldsymbol{a}, \boldsymbol{c}|}=-1$ :

$$
\begin{aligned}
-\sum_{\boldsymbol{b} \in \mathfrak{K}}(-1)^{|\boldsymbol{a}, \boldsymbol{b}|} & =\sum_{\boldsymbol{b} \in \mathfrak{K}}(-1)^{|\boldsymbol{a}, \boldsymbol{b}|+|\boldsymbol{a}, \boldsymbol{c}|}=\sum_{\boldsymbol{b} \in \mathfrak{K}}(-1)^{t} \boldsymbol{a}^{\prime}\left(\boldsymbol{b}^{\prime \prime}+\boldsymbol{c}^{\prime \prime}\right)-{ }^{t}\left(\boldsymbol{b}^{\prime}+\boldsymbol{c}^{\prime}\right) \boldsymbol{a}^{\prime \prime} \\
& =\sum_{\boldsymbol{b} \in \mathfrak{K}}(-1)^{|\boldsymbol{a}, \boldsymbol{b}+\boldsymbol{c}|}=\sum_{\boldsymbol{b} \in \mathfrak{K}}(-1)^{|\boldsymbol{a}, \boldsymbol{b}|}
\end{aligned}
$$

(мы изменили порядок суммирования $\boldsymbol{b}+\boldsymbol{c} \mapsto \boldsymbol{b}$ ). Правая и левая части равенства (3.5) отличаются зна́ком, и это завершает доказательство тождеств (3.4). 
Лемма 7. Справедливы тождества

$$
\sum_{\boldsymbol{b} \in \mathfrak{K}^{*}}(-1)^{|\boldsymbol{a}, \boldsymbol{b}|}=\left\{\begin{array}{ll}
2^{g-1}\left(2^{g}+1\right), & \text { если } \boldsymbol{a}=\mathbf{0}, \\
(-1)^{|\boldsymbol{a}|} \cdot 2^{g-1} & \text { в противном случае, }
\end{array} \quad \boldsymbol{a} \in \mathfrak{K} .\right.
$$

ДоКАЗАТЕЛЬСТво. В случае $\boldsymbol{a}=\mathbf{0}$ все слагаемые в левой части равны 1 , т.е. сумма равна количеству слагаемых. Пусть $\boldsymbol{a} \neq \mathbf{0}$. Рассмотрим суммы

$$
S_{+}=\sum_{\boldsymbol{b}:|\boldsymbol{b}| \text { четно }}(-1)^{|\boldsymbol{a}, \boldsymbol{b}|}, \quad S_{-}=-\sum_{\boldsymbol{b}:|\boldsymbol{b}| \text { нечетно }}(-1)^{|\boldsymbol{a}, \boldsymbol{b}|} .
$$

Согласно лемме $6 S_{+}-S_{-}=0$, т.е. $S_{+}=S_{-}$. Заметим теперь, что

$$
\begin{aligned}
& (-1)^{|\boldsymbol{a}, \boldsymbol{b}|}=(-1)^{{ }^{t} \boldsymbol{a}^{\prime} \boldsymbol{b}^{\prime \prime}-{ }^{t} \boldsymbol{b}^{\prime} \boldsymbol{a}^{\prime \prime}}=(-1)^{t}\left(\boldsymbol{a}^{\prime}+\boldsymbol{b}^{\prime}\right)\left(\boldsymbol{a}^{\prime \prime}+\boldsymbol{b}^{\prime \prime}\right)(-1)^{{ }^{t} \boldsymbol{a}^{\prime} \boldsymbol{a}^{\prime \prime}}(-1)^{{ }^{t} \boldsymbol{b}^{\prime} \boldsymbol{b}^{\prime \prime}} \\
& =(-1)^{|\boldsymbol{a}+\boldsymbol{b}|}(-1)^{|\boldsymbol{a}|}(-1)^{|\boldsymbol{b}|} .
\end{aligned}
$$

Поэтому суммы (3.7) можно переписать в виде

$$
S_{+}=(-1)^{|\boldsymbol{a}|} \sum_{\boldsymbol{b}:|\boldsymbol{b}| \text { четно }}(-1)^{|\boldsymbol{a}+\boldsymbol{b}|}, \quad S_{-}=(-1)^{|\boldsymbol{a}|} \sum_{\boldsymbol{b}:|\boldsymbol{b}| \text { нечетно }}(-1)^{|\boldsymbol{a}+\boldsymbol{b}|} .
$$

Следовательно,

$$
\begin{aligned}
S_{+}+S_{-} & =(-1)^{|\boldsymbol{a}|} \sum_{\boldsymbol{b} \in \mathfrak{K}}(-1)^{|\boldsymbol{a}+\boldsymbol{b}|}=(-1)^{|\boldsymbol{a}|} \sum_{\boldsymbol{b} \in \mathfrak{K}}(-1)^{|\boldsymbol{b}|} \\
& =(-1)^{|\boldsymbol{a}|}\left(\sum_{\boldsymbol{b}:|\boldsymbol{b}| \text { четно }} 1-\sum_{\boldsymbol{b}:|\boldsymbol{b}| \text { нечетно }} 1\right) \\
& =(-1)^{|\boldsymbol{a}|}\left(2^{g-1}\left(2^{g}+1\right)-2^{2 g}+2^{g-1}\left(2^{g}+1\right)\right)=(-1)^{|\boldsymbol{a}|} \cdot 2^{g} .
\end{aligned}
$$

Таким образом, $S_{+}=S_{-}=(-1)^{|\boldsymbol{a}|} \cdot 2^{g-1}$, что завершает доказательство тождеств (3.6).

Лемма 8. Матрица М удовлетворяет соотношению

$$
\mathrm{M}^{2}=\frac{1}{2}(\mathrm{M}+E)
$$

где $E$ - единичная матрица размера $2^{g-1}\left(2^{g}+1\right)$.

ДокАЗАТЕЛЬСтво. Пользуясь леммой 7 , рассмотрим скалярное произведение строк матрицы М с номерами $\boldsymbol{a}$ и $\boldsymbol{c}$ :

$$
\begin{aligned}
\frac{1}{2^{2 g}} & \sum_{\boldsymbol{b} \in \mathfrak{K}^{*}}(-1)^{|\boldsymbol{a}, \boldsymbol{b}|} \cdot(-1)^{|\boldsymbol{c}, \boldsymbol{b}|}=\frac{1}{2^{2 g}} \sum_{\boldsymbol{b} \in \mathfrak{K}^{*}}(-1)^{|\boldsymbol{a}-\boldsymbol{c}, \boldsymbol{b}|} \\
& =\left\{\begin{array}{l}
2^{-g-1}\left(2^{g}+1\right), \quad \text { если } \boldsymbol{a}=\boldsymbol{c}, \\
(-1)^{|\boldsymbol{a}-\boldsymbol{c}|} \cdot 2^{-g-1} \quad \text { в противном случае, }
\end{array}\right. \\
& =\left\{\begin{array}{l}
\frac{1}{2^{g+1}}+\frac{1}{2}, \quad \text { если } \boldsymbol{a}=\boldsymbol{c}, \\
\frac{1}{2^{g+1}}(-1)^{|\boldsymbol{a}, \boldsymbol{c}|} \quad \text { в противном случае. }
\end{array}\right.
\end{aligned}
$$

В силу симметричности матрицы М (произведение строк равно произведению строки на столбец) соотношения (3.9) в точности означают справедливость матричного тождества (3.8). 
СледСтвиЕ. Справедливо тождество

$$
\left(\mathrm{M}-\frac{1}{4} E\right)^{-1}=\frac{16}{9}\left(\mathrm{M}-\frac{1}{4} E\right) .
$$

ДокАЗАТЕЛьство. Действительно, согласно лемме 8 имеем

$$
\left(\mathrm{M}-\frac{1}{4} E\right)^{2}=\mathrm{M}^{2}-\frac{1}{2} \mathrm{M}+\frac{1}{16} E=\frac{9}{16} E,
$$

откуда следует тождество (3.10).

ЗАмЕчАниЕ. В дальнейшем мы воспользуемся схемой доказательства леммы 8, поэтому мы предпочли его подробно проделать, не прибегая к готовьм свойствам матрицы М из литературы (см., например, [24; § 2]).

Поскольку матрица М симметрична, ее жорданова нормальная форма имеет диагональный вид. Поэтому все собственные значения $\lambda$ матрицы М удовлетворяют квадратному уравнению (3.8):

$$
\lambda^{2}=\frac{1}{2}(\lambda+1)
$$

откуда $\lambda=1$ или $\lambda=-\frac{1}{2}$.

ЛЕмма 9. Кратность собственных значений $\lambda_{1}=1$ и $\lambda_{2}=-\frac{1}{2}$ в характеристическом многочлене матрииы М равна

$$
s_{1}=\frac{\left(2^{g-1}+1\right)\left(2^{g}+1\right)}{3} \quad u \quad s_{2}=\frac{\left(2^{g}-1\right)\left(2^{g}+1\right)}{3}
$$

соответственно.

ДокАЗАтЕльство. Это утверждение является легким упражнением по линейной алгебре. Если $s_{1}$ и $s_{2}-$ кратности корней $\lambda_{1}=1$ и $\lambda_{2}=-\frac{1}{2}$ соответственно (других корней у характеристического многочлена матрицы М нет), то $s_{1}+s_{2}=2^{g-1}\left(2^{g}+1\right)$. След матрицы инвариантен при переходе к жордановой нормальной форме. Жорданова форма симметричной матрицы М диагональная матрица, на диагонали $s_{1}$ раз стоит $\lambda_{1}$ и $s_{2}$ раз $\lambda_{2}$. Поэтому $\operatorname{tr} \mathrm{M}=s_{1} \lambda_{1}+s_{2} \lambda_{2}=s_{1}-\frac{1}{2} s_{2}$. С другой стороны, на диагонали матрицы М стоят числа $2^{-g}$, т.е. $\operatorname{tr} \mathrm{M}=2^{-g} \cdot 2^{g-1}\left(2^{g}+1\right)=\frac{1}{2}\left(2^{g}+1\right)$. Решая систему линейных уравнений

$$
s_{1}+s_{2}=2^{g-1}\left(2^{g}+1\right), \quad s_{1}-\frac{1}{2} s_{2}=\frac{1}{2}\left(2^{g}+1\right),
$$

находим значения (3.11). Лемма доказана. 


\section{§4. Доказательство теоремы 1}

С помощью леммы 2 запишем разложения четных тэта-функций, стоящих в левой и правой частях тождеств леммы 5 :

$$
\begin{aligned}
\vartheta_{\boldsymbol{a}}^{4}(\boldsymbol{z}) & =\vartheta_{\boldsymbol{a}}^{4} \cdot\left(1-2 \psi_{\boldsymbol{a}}+\frac{2}{3} \psi_{\boldsymbol{a}}^{2}+\frac{2}{3} \delta \psi_{\boldsymbol{a}}\right)^{4}+O\left(\boldsymbol{z}^{6}\right) \\
& =\vartheta_{\boldsymbol{a}}^{4} \cdot\left(1-8 \psi_{\boldsymbol{a}}+\frac{80}{3} \psi_{\boldsymbol{a}}^{2}+\frac{8}{3} \delta \psi_{\boldsymbol{a}}\right)+O\left(\boldsymbol{z}^{6}\right), \quad \boldsymbol{a} \in \mathfrak{K}^{*}, \\
\vartheta_{\boldsymbol{b}}(2 \boldsymbol{z}) & =\vartheta_{\boldsymbol{b}} \cdot\left(1-8 \psi_{\boldsymbol{b}}+\frac{32}{3} \psi_{\boldsymbol{b}}^{2}+\frac{32}{3} \delta \psi_{\boldsymbol{b}}\right)+O\left(\boldsymbol{z}^{6}\right), \quad \boldsymbol{b} \in \mathfrak{K}^{*} .
\end{aligned}
$$

Подставляя (4.1) в тождества (3.3) и сравнивая однородные компоненты степени 4 получающихся разложений, приходим к формулам

$$
\vartheta_{\boldsymbol{a}}^{4} \cdot \frac{1}{3}\left(80 \psi_{\boldsymbol{a}}^{2}+8 \delta \psi_{\boldsymbol{a}}\right)=\frac{1}{2^{g}} \sum_{\boldsymbol{b} \in \mathfrak{K}^{*}}(-1)^{|\boldsymbol{a}, \boldsymbol{b}|} \vartheta_{\boldsymbol{b}}^{4} \cdot \frac{1}{3}\left(32 \psi_{\boldsymbol{b}}^{2}+32 \delta \psi_{\boldsymbol{b}}\right), \quad \boldsymbol{a} \in \mathfrak{K}^{*}
$$

Перепишем соотношения (4.2) в матричном виде. Для этого мы рассмотрим векторы (столбцы)

$$
\boldsymbol{X}=\left(\vartheta_{\boldsymbol{a}}^{4} \cdot \psi_{\boldsymbol{a}}^{2}\right)_{\boldsymbol{a} \in \mathfrak{K}^{*}}, \quad \boldsymbol{Y}=\left(\vartheta_{\boldsymbol{a}}^{4} \cdot \delta \psi_{\boldsymbol{a}}\right)_{\boldsymbol{a} \in \mathfrak{K}^{*}}
$$

В обозначениях (4.3) соотношения (4.2) после упрощения примут вид

$$
\frac{5}{2} \boldsymbol{X}+\frac{1}{4} \boldsymbol{Y}=\mathrm{M}(\boldsymbol{X}+\boldsymbol{Y})
$$

откуда

$$
\begin{aligned}
\boldsymbol{Y} & =-\left(\mathrm{M}-\frac{1}{4} E\right)^{-1}\left(\mathrm{M}-\frac{5}{2} E\right) \boldsymbol{X}=-\boldsymbol{X}+\frac{9}{4}\left(\mathrm{M}-\frac{1}{4} E\right)^{-1} \boldsymbol{X} \\
& =-\boldsymbol{X}+4\left(\mathrm{M}-\frac{1}{4} E\right) \boldsymbol{X}=(4 \mathrm{M}-2 E) \boldsymbol{X}
\end{aligned}
$$

где мы воспользовались леммой 8 и следствием из нее.

Соотношения (4.4) после декодирования (4.3) дают систему дифференциальных уравнений (0.20). Система (0.17) следует из нее и соотношений (2.1) леммы 2.

Теорема 1 доказана.

\section{§5. Дальнейшее обобщение теоремы 1}

Доказьвая (не самым простьм способом) теорему Альфена, мы фактически доказали одномерный вариант теоремы 1 (система (1.7)) и, кроме того, воспользовались соотношениями между тэта-константами и функциями (0.18), получающимися сравнением постоянных и квадратичных коэффициентов. Последняя операция имеет многомерньй аналог: ничто не мешает нам сравнить однородные компоненты степени 0 и 2 после подстановки разложений (4.1) в тождества (3.3):

$$
\vartheta_{\boldsymbol{a}}^{4}=\frac{1}{2^{g}} \sum_{\boldsymbol{b} \in \mathfrak{K}_{0}}(-1)^{|\boldsymbol{a}, \boldsymbol{b}|} \vartheta_{\boldsymbol{b}}^{4}, \quad \vartheta_{\boldsymbol{a}}^{4} \psi_{\boldsymbol{a}}=\frac{1}{2^{g}} \sum_{\boldsymbol{b} \in \mathfrak{K}_{0}}(-1)^{|\boldsymbol{a}, \boldsymbol{b}|} \vartheta_{\boldsymbol{b}}^{4} \psi_{\boldsymbol{b}}, \quad \boldsymbol{a} \in \mathfrak{K}_{0} .
$$


Однако, получающиеся выражения для всевозможных отношений тэта-констант имеют далеко не такой простой вид, как формулы (1.8). А как выглядит система уравнений для $\psi$-функций, если воспользоваться схемой простого доказательства теоремы Альфена? Ответу на этот вопрос и посвящен $\S 5$.

Зафиксируем характеристику $\boldsymbol{c} \in \mathfrak{K}, \boldsymbol{c} \neq \mathbf{0}$, и вернемся к соотношениям Римана $(3.2)$, полагая в них на этот раз $\boldsymbol{d}=\mathbf{0}$ :

$$
\begin{gathered}
(-1)^{t}{ }^{\boldsymbol{c}^{\prime} \boldsymbol{a}^{\prime \prime}} \vartheta_{\boldsymbol{a}+\boldsymbol{c}}^{2}(\boldsymbol{z}) \vartheta_{\boldsymbol{a}}^{2}(\boldsymbol{z})=\frac{1}{2^{g}} \sum_{\boldsymbol{b} \in \mathfrak{K}}(-1)^{|\boldsymbol{a}, \boldsymbol{b}|}(-1)^{t \boldsymbol{c}^{\prime} \boldsymbol{b}^{\prime \prime}} \vartheta_{\boldsymbol{b}+\boldsymbol{c}}(2 \boldsymbol{z}) \vartheta_{\boldsymbol{b}+\boldsymbol{c}}(\mathbf{0}) \vartheta_{\boldsymbol{b}}^{2}(\mathbf{0}), \\
\boldsymbol{a} \in \mathfrak{K} .
\end{gathered}
$$

Заметим, что теперь суммирование в правой части равенств (5.2) происходит только по тем характеристикам $\boldsymbol{b}$, для которых $\boldsymbol{b}$ и $\boldsymbol{b}+\boldsymbol{c}$ являются четными. Характеристике $\boldsymbol{c} \in \mathfrak{K} \backslash\{\mathbf{0}\}$ отвечает подгруппа $\{\mathbf{0}, \boldsymbol{c}\} \subset \mathfrak{K}$ порядка 2. Элементы $(\boldsymbol{a}, \boldsymbol{a}+\boldsymbol{c})$ факторгруппы $\mathfrak{K}_{\boldsymbol{c}}=\mathfrak{K} /\{\boldsymbol{c}\}$ мы также будем называть характеристиками, отождествляя их с любым представителем такой пары; четными при этом будут те и только те характеристики, для которых и $\boldsymbol{a}$ и $\boldsymbol{a}+\boldsymbol{c}$ четны. Множество четных характеристик групшы $\mathfrak{K}_{c}$ мы обозначим через $\mathfrak{K}_{c}^{*}$. В дальнейшем, выбирая и фиксируя по одному представителю пары $(\boldsymbol{a}, \boldsymbol{a}+\boldsymbol{c}) \in \mathfrak{K}_{\boldsymbol{c}}$, мы будем отождествлять группу $\mathfrak{K}_{\boldsymbol{c}}$ и ее подмножество $\mathfrak{K}_{\boldsymbol{c}}^{*}$ с подмножествами в $\mathfrak{K}$.

Полагая

$$
\begin{aligned}
& \theta_{\boldsymbol{a}}(\boldsymbol{z})=i^{{ }^{t} \boldsymbol{c}^{\prime} \boldsymbol{a}^{\prime \prime}} \vartheta_{\boldsymbol{a}+\boldsymbol{c}}(\boldsymbol{z}) \vartheta_{\boldsymbol{a}}(\boldsymbol{z}), \\
& \tilde{\theta}_{\boldsymbol{a}}(\boldsymbol{z})=i^{{ }^{t} \boldsymbol{c}^{\prime} \boldsymbol{a}^{\prime \prime}} \frac{1}{2}\left(\vartheta_{\boldsymbol{a}+\boldsymbol{c}}(2 \boldsymbol{z}) \vartheta_{\boldsymbol{a}}(\mathbf{0})+\vartheta_{\boldsymbol{a}+\boldsymbol{c}}(\mathbf{0}) \vartheta_{\boldsymbol{a}}(2 \boldsymbol{z})\right) \\
& \theta_{\boldsymbol{a}}=\theta_{\boldsymbol{a}}(\mathbf{0})=\tilde{\theta}_{\boldsymbol{a}}(\mathbf{0}), \\
& a \in \mathfrak{K}_{c}^{*},
\end{aligned}
$$

и группируя слагаемые, отвечающие характеристикам $\boldsymbol{b}$ и $\boldsymbol{b}+\boldsymbol{c}$, перепишем соотношения (5.2) в виде

$$
\theta_{\boldsymbol{a}}^{2}(\boldsymbol{z})=\frac{1}{2^{g-1}} \sum_{\boldsymbol{b} \in \mathfrak{K}_{\boldsymbol{c}}^{*}}(-1)^{|\boldsymbol{a}, \boldsymbol{b}|} \widetilde{\theta}_{\boldsymbol{b}}(\boldsymbol{z}) \theta_{\boldsymbol{b}}, \quad \boldsymbol{a} \in \mathfrak{K}_{\boldsymbol{c}}^{*} .
$$

Наряду с формулами (5.4) нам понадобится также еще одна “вырожденная" специализация соотношений Римана:

$$
\theta_{\boldsymbol{a}}(\boldsymbol{z}) \theta_{\boldsymbol{a}}=\frac{1}{2^{g-1}} \sum_{\boldsymbol{b} \in \mathfrak{K}_{c}^{*}}(-1)^{|\boldsymbol{a}, \boldsymbol{b}|} \theta_{\boldsymbol{b}}(\boldsymbol{z}) \theta_{\boldsymbol{b}}, \quad \boldsymbol{a} \in \mathfrak{K}_{\boldsymbol{c}}^{*},
$$

которая получается после подстановки $\boldsymbol{d}=\mathbf{0}, \boldsymbol{z}_{1}=\boldsymbol{z}_{3}=\boldsymbol{z}, \boldsymbol{z}_{2}=\boldsymbol{z}_{4}=\mathbf{0}$ в соотношения (3.1) и группирования в правой части слагаемых, отвечающих характеристикам $\boldsymbol{b}$ и $\boldsymbol{b}+\boldsymbol{c}$.

Как и в преддверии доказательства теоремы 1, мы ознакомимся со свойствами симметричной матрицы

$$
\mathrm{M}=\mathrm{M}_{\boldsymbol{c}}=\frac{1}{2^{g-1}}\left((-1)^{|\boldsymbol{a}, \boldsymbol{b}|}\right)_{\boldsymbol{a}, \boldsymbol{b} \in \mathfrak{K}_{c}^{*}}
$$

(неизвестного пока размера), отвечаюшей тождествам (5.4) и (5.5). 
Прежде всего, вычислим некоторые вспомогательные суммы.

Лемма 10. Справедливъ тождества

$$
\sum_{\boldsymbol{b} \in \mathfrak{K}^{*}: \boldsymbol{b}+\boldsymbol{c} \in \mathfrak{K}^{*}}(-1)^{|\boldsymbol{a}, \boldsymbol{b}|}=(-1)^{|\boldsymbol{a}|} \cdot \begin{cases}2^{g-1}\left(2^{g-1}+1\right), & \text { если } \boldsymbol{a}=\mathbf{0} \text { или } \boldsymbol{a}=\boldsymbol{c}, \\ \left((-1)^{|\boldsymbol{a}, \boldsymbol{c}|}+1\right) \cdot 2^{g-2} & \text { в остальных случаях, } \\ \boldsymbol{a} \in \mathfrak{K} . & (5.6)\end{cases}
$$

ДокАЗАТЕЛьство. Для заданной характеристики $\boldsymbol{a} \in \mathfrak{K}$ обозначим через $S_{+}$ сумму в правой части соотношений (5.6) и наряду с ней рассмотрим сумму

$$
S_{-}=-\sum_{\boldsymbol{b} \in \mathfrak{K}^{*}: \boldsymbol{b}+\boldsymbol{c} \notin \mathfrak{K}^{*}}(-1)^{|\boldsymbol{a}, \boldsymbol{b}|} .
$$

Согласно лемме 7

$$
S_{+}-S_{-}=(-1)^{|\boldsymbol{a}|} \cdot \begin{cases}2^{g-1}\left(2^{g}+1\right), & \text { если } \boldsymbol{a}=\mathbf{0}, \\ 2^{g-1} & \text { в противном случае. }\end{cases}
$$

Поскольку

$$
(-1)^{|\boldsymbol{a}, \boldsymbol{b}|}=(-1)^{|\boldsymbol{c}|}(-1)^{|\boldsymbol{b}|}(-1)^{|\boldsymbol{b}+\boldsymbol{c}|}(-1)^{|\boldsymbol{a}+\boldsymbol{c}, \boldsymbol{b}|},
$$

суммы $S_{+}$и $S_{-}$можно переписать в виде

$$
S_{+}=(-1)^{|\boldsymbol{c}|} \sum_{\boldsymbol{b} \in \mathfrak{K}^{*}: \boldsymbol{b}+\boldsymbol{c} \in \mathfrak{K}^{*}}(-1)^{|\boldsymbol{a}+\boldsymbol{c}, \boldsymbol{b}|}, \quad S_{-}=(-1)^{|\boldsymbol{c}|} \sum_{\boldsymbol{b} \in \mathfrak{K}^{*}: \boldsymbol{b}+\boldsymbol{c} \notin \mathfrak{K}^{*}}(-1)^{|\boldsymbol{a}+\boldsymbol{c}, \boldsymbol{b}|} .
$$

Следовательно,

$$
\begin{aligned}
S_{+}+S_{-} & =(-1)^{|\boldsymbol{c}|} \sum_{\boldsymbol{b} \in \mathfrak{K}^{*}}(-1)^{|\boldsymbol{a}+\boldsymbol{c}, \boldsymbol{b}|} \\
& =(-1)^{|\boldsymbol{c}|} \cdot \begin{cases}2^{g-1}\left(2^{g}+1\right), & \text { если } \boldsymbol{a}=\boldsymbol{c}, \\
(-1)^{|\boldsymbol{a}+\boldsymbol{c}|} \cdot 2^{g-1} & \text { в противном случае, }\end{cases}
\end{aligned}
$$

где мы снова воспользовались леммой 7 .

Складывая равенства $(5.7)$ и $(5.8)$, находим $S_{+}$равньп правой части тождества (5.6). Лемма доказана.

СлЕДСТВИЕ. В множестве $\mathfrak{K}_{\boldsymbol{c}}^{*}$ содержится $2^{g-2}\left(2^{g-1}+1\right)$ әлементов.

ДокАЗАТЕЛЬСтво. Рассматривая тождество (5.6) при $\boldsymbol{a}=\mathbf{0}$, в правой части получаем количество элементов множества $\left\{\boldsymbol{b} \in \mathfrak{K}^{*}: \boldsymbol{b}+\boldsymbol{c} \in \mathfrak{K}^{*}\right\}$. Остается разделить это число пополам.

Лемма 11. Матрица $\mathrm{M}=\mathrm{M}_{\boldsymbol{c}}$ удовлетворяет соотношению

$$
\mathrm{M}^{2}=\frac{1}{2}(\mathrm{M}+E),
$$

әде $E-$ единичная матрица размера $2^{g-2}\left(2^{g-1}+1\right)$. 
ДокАЗАТЕЛЬСтво. Пользуясь леммой 10, рассмотрим скалярное произведение строк матрицы М с номерами $\boldsymbol{a}, \boldsymbol{d} \in \mathfrak{K}_{\boldsymbol{c}}^{*}\left(\right.$ случай $\boldsymbol{a}+\boldsymbol{c} \equiv \boldsymbol{d}\left(\bmod 2 \mathbb{Z}^{2 g}\right)$ согласно определению множества $\mathfrak{K}_{\boldsymbol{c}}^{*}$ невозможен!):

$$
\begin{aligned}
& \sum_{\boldsymbol{b} \in \mathfrak{K}_{\boldsymbol{c}}^{*}} \frac{1}{2^{g-1}}(-1)^{|\boldsymbol{a}, \boldsymbol{b}|} \cdot \frac{1}{2^{g-1}}(-1)^{|\boldsymbol{d}, \boldsymbol{b}|}=\frac{1}{2^{2 g-1}} \sum_{\boldsymbol{b} \in \mathfrak{K}^{*}: \boldsymbol{b}+\boldsymbol{c} \in \mathfrak{K}^{*}}(-1)^{|\boldsymbol{a}+\boldsymbol{d}, \boldsymbol{b}|} \\
& =\frac{1}{2^{2 g-1}} \cdot\left\{\begin{array}{l}
2^{g-1}\left(2^{g-1}+1\right), \\
(-1)^{|\boldsymbol{a}+\boldsymbol{d}|} \cdot 2^{g-1}
\end{array}\right. \\
& \quad=\frac{1}{2} \cdot \begin{cases}\frac{1}{2^{g-1}}+\frac{1}{2}, & \text { в прли } \boldsymbol{a}=\boldsymbol{d}, \\
\frac{1}{2^{g-1}}(-1)^{|\boldsymbol{a}, \boldsymbol{d}|} & \text { в противном случае, } \boldsymbol{a}=\boldsymbol{d},\end{cases}
\end{aligned}
$$

С учетом симметричности матрицы М (произведение строк равно произведению строки на столбец) лемма доказана.

СлЕДСТВИЕ 1. Справедливо тождество

$$
\left(\mathrm{M}-\frac{1}{4} E\right)^{-1}=\frac{16}{9}\left(\mathrm{M}-\frac{1}{4} E\right) .
$$

ДоКАЗАТЕЛЬСТво повторяет доказательство следствия из леммы 8.

СлЕДСТВИЕ 2. Матрица

$$
(\mathrm{M}-E)\left(\mathrm{M}+\frac{1}{2} E\right)
$$

нулевая.

ДокАЗАтЕльство. Данная матрица представляет собой разложение матрицы $\mathrm{M}^{2}-\frac{1}{2}(\mathrm{M}+E)$ на линейные множители.

Теперь по традиции следовало бы рассмотреть кратность собственных значений 1 и $-\frac{1}{2}$ в характеристическом многочлене матрицы М. Мы предоставляем это читателю в качестве простого (особенно после доказанной леммы 9) упражнения.

ТЕОрема 2. Для заданной характеристики $\boldsymbol{c} \in \mathfrak{K}, \boldsymbol{c} \neq \mathbf{0}$, и отвечающего ей множсества $\mathfrak{K}_{c}^{*}$ функиии (0.18) удовлетворяют системе дифференциальньх уравнений

$$
\begin{aligned}
& \vartheta_{\boldsymbol{a}+\boldsymbol{c}}^{2} \vartheta_{\boldsymbol{a}}^{2} \delta\left(\psi_{\boldsymbol{a}+\boldsymbol{c}}+\psi_{\boldsymbol{a}}\right) \\
& =\frac{1}{2^{g-2}} \sum_{\boldsymbol{b} \in \mathfrak{K}_{\boldsymbol{c}}^{*}}(-1)^{|\boldsymbol{a}, \boldsymbol{b}|}(-1)^{t} \boldsymbol{c}^{\prime}\left(\boldsymbol{a}^{\prime \prime}+\boldsymbol{b}^{\prime \prime}\right) \vartheta_{\boldsymbol{b}+\boldsymbol{c}}^{2} \vartheta_{\boldsymbol{b}}^{2}\left(\psi_{\boldsymbol{b}+\boldsymbol{c}}+\psi_{\boldsymbol{b}}\right)^{2} \\
& +4 \vartheta_{\boldsymbol{a}+\boldsymbol{c}}^{2} \vartheta_{\boldsymbol{a}}^{2} \psi_{\boldsymbol{a}+\boldsymbol{c}} \psi_{\boldsymbol{a}}-2 \vartheta_{\boldsymbol{a}+\boldsymbol{c}}^{2} \vartheta_{\boldsymbol{a}}^{2}\left(\psi_{\boldsymbol{a}+\boldsymbol{c}}+\psi_{\boldsymbol{a}}\right)^{2}, \quad \boldsymbol{a} \in \mathfrak{K}_{\boldsymbol{c}}^{*} .
\end{aligned}
$$

Кроме того, тэта-константы и функиии (0.18) связаны соотношениями

$$
\begin{aligned}
& \vartheta_{\boldsymbol{a}+\boldsymbol{c}}^{2} \vartheta_{\boldsymbol{a}}^{2}=\frac{1}{2^{g-1}} \sum_{\boldsymbol{b} \in \mathfrak{K}_{c}^{*}}(-1)^{|\boldsymbol{a}, \boldsymbol{b}|}(-1)^{t} \boldsymbol{c}^{\prime}\left(\boldsymbol{a}^{\prime \prime}+\boldsymbol{b}^{\prime \prime}\right) \vartheta_{\boldsymbol{b}+\boldsymbol{c}}^{2} \vartheta_{\boldsymbol{b}}^{2}, \quad \boldsymbol{a} \in \underset{\mathfrak{K}_{\boldsymbol{c}}^{*}}{(5.10)}
\end{aligned}
$$

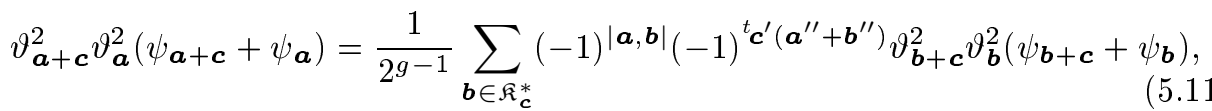

$$
\begin{aligned}
& a \in \mathfrak{K}_{c}^{*} .
\end{aligned}
$$


ДокАЗАТЕЛьство. Согласно лемме 2 имеем

$$
\begin{aligned}
& \theta_{\boldsymbol{a}}(\boldsymbol{z})=i^{{ }^{t} \boldsymbol{c}^{\prime} \boldsymbol{a}^{\prime \prime}} \vartheta_{\boldsymbol{a}+\boldsymbol{c}}(\boldsymbol{z}) \vartheta_{\boldsymbol{a}}(\boldsymbol{z})=\theta_{\boldsymbol{a}} \cdot\left(1-2\left(\psi_{\boldsymbol{a}+\boldsymbol{c}}+\psi_{\boldsymbol{a}}\right)\right. \\
& \left.+\frac{8}{3} \psi_{\boldsymbol{a}+\boldsymbol{c}} \psi_{\boldsymbol{a}}+\frac{2}{3}\left(\psi_{\boldsymbol{a}+\boldsymbol{c}}+\psi_{\boldsymbol{a}}\right)^{2}+\frac{2}{3} \delta\left(\psi_{\boldsymbol{a}+\boldsymbol{c}}+\psi_{\boldsymbol{a}}\right)\right)+O\left(\boldsymbol{z}^{6}\right), \\
& \theta_{\boldsymbol{a}}^{2}(\boldsymbol{z})=(-1)^{t \boldsymbol{c}^{\prime} \boldsymbol{a}^{\prime \prime}} \vartheta_{\boldsymbol{a}+\boldsymbol{c}}^{2}(\boldsymbol{z}) \vartheta_{\boldsymbol{a}}^{2}(\boldsymbol{z})=\theta_{\boldsymbol{a}}^{2} \cdot\left(1-4\left(\psi_{\boldsymbol{a}+\boldsymbol{c}}+\psi_{\boldsymbol{a}}\right)\right. \\
& \left.\quad+\frac{16}{3} \psi_{\boldsymbol{a}+\boldsymbol{c}} \psi_{\boldsymbol{a}}+\frac{16}{3}\left(\psi_{\boldsymbol{a}+\boldsymbol{c}}+\psi_{\boldsymbol{a}}\right)^{2}+\frac{4}{3} \delta\left(\psi_{\boldsymbol{a}+\boldsymbol{c}}+\psi_{\boldsymbol{a}}\right)\right)+O\left(\boldsymbol{z}^{6}\right), \\
& \tilde{\theta}_{\boldsymbol{a}}(\boldsymbol{z})=i^{{ }^{\prime} \boldsymbol{c}^{\prime} \boldsymbol{a}^{\prime \prime}} \frac{1}{2}\left(\vartheta_{\boldsymbol{a}+\boldsymbol{c}}(2 \boldsymbol{z}) \vartheta_{\boldsymbol{a}}+\vartheta_{\boldsymbol{a}+\boldsymbol{c}} \vartheta_{\boldsymbol{a}}(2 \boldsymbol{z})\right)=\theta_{\boldsymbol{a}} \cdot\left(1-4\left(\psi_{\boldsymbol{a}+\boldsymbol{c}}+\psi_{\boldsymbol{a}}\right)\right. \\
& \left.\quad-\frac{32}{3} \psi_{\boldsymbol{a}+\boldsymbol{c}} \psi_{\boldsymbol{a}}+\frac{16}{3}\left(\psi_{\boldsymbol{a}+\boldsymbol{c}}+\psi_{\boldsymbol{a}}\right)^{2}+\frac{16}{3} \delta\left(\psi_{\boldsymbol{a}+\boldsymbol{c}}+\psi_{\boldsymbol{a}}\right)\right)+O\left(\boldsymbol{z}^{6}\right),
\end{aligned}
$$$$
a \in \mathfrak{K}_{c}^{*} .
$$

Учитывая $\theta_{\boldsymbol{a}}^{2}=(-1)^{t} \boldsymbol{c}^{\prime} \boldsymbol{a}^{\prime \prime} \vartheta_{\boldsymbol{a}+\boldsymbol{c}}^{2} \vartheta_{\boldsymbol{a}}^{2}, \boldsymbol{a} \in \mathfrak{K}_{\boldsymbol{c}}^{*}$, подставляя разложения (5.12) в тождества $(5.4),(5.5)$ и сравнивая однородные компоненты степени 0,2 и 4, получаем соответственно соотношения (5.10), (5.11) и

$$
\begin{gathered}
\theta_{\boldsymbol{a}}^{2}\left(4 \psi_{\boldsymbol{a}+\boldsymbol{c}} \psi_{\boldsymbol{a}}+4\left(\psi_{\boldsymbol{a}+\boldsymbol{c}}+\psi_{\boldsymbol{a}}\right)^{2}+\delta\left(\psi_{\boldsymbol{a}+\boldsymbol{c}}+\psi_{\boldsymbol{a}}\right)\right) \\
=\frac{1}{2^{g-1}} \sum_{\boldsymbol{b} \in \mathfrak{K}_{\boldsymbol{c}}^{*}}(-1)^{|\boldsymbol{a}, \boldsymbol{b}|} \cdot 4 \theta_{\boldsymbol{b}}^{2}\left(-2 \psi_{\boldsymbol{b}+\boldsymbol{c}} \psi_{\boldsymbol{b}}+\left(\psi_{\boldsymbol{b}+\boldsymbol{c}}+\psi_{\boldsymbol{b}}\right)^{2}+\delta\left(\psi_{\boldsymbol{b}+\boldsymbol{c}}+\psi_{\boldsymbol{b}}\right)\right) \\
\theta_{\boldsymbol{a}}^{2}\left(4 \psi_{\boldsymbol{a}+\boldsymbol{c}} \psi_{\boldsymbol{a}}+\left(\psi_{\boldsymbol{a}+\boldsymbol{c}}+\psi_{\boldsymbol{a}}\right)^{2}+\delta\left(\psi_{\boldsymbol{a}+\boldsymbol{c}}+\psi_{\boldsymbol{a}}\right)\right) \\
=\frac{1}{2^{g-1}} \sum_{\boldsymbol{b} \in \mathfrak{K}_{\boldsymbol{c}}^{*}}(-1)^{|\boldsymbol{a}, \boldsymbol{b}|} \theta_{\boldsymbol{b}}^{2}\left(4 \psi_{\boldsymbol{b}+\boldsymbol{c}} \psi_{\boldsymbol{b}}+\left(\psi_{\boldsymbol{b}+\boldsymbol{c}}+\psi_{\boldsymbol{b}}\right)^{2}+\delta\left(\psi_{\boldsymbol{b}+\boldsymbol{c}}+\psi_{\boldsymbol{b}}\right)\right) \\
\boldsymbol{a} \in \mathfrak{K}_{\boldsymbol{c}}^{*},
\end{gathered}
$$

По аналогии с доказательством теоремы 1 рассмотрим векторы (столбцы)

$$
\begin{gathered}
\boldsymbol{W}=\left(\theta_{\boldsymbol{a}}^{2} \psi_{\boldsymbol{a}+\boldsymbol{c}} \psi_{\boldsymbol{a}}\right)_{\boldsymbol{a} \in \mathfrak{K}_{\boldsymbol{c}}^{*}}, \quad \boldsymbol{X}=\left(\theta_{\boldsymbol{a}}^{2}\left(\psi_{\boldsymbol{a}+\boldsymbol{c}}+\psi_{\boldsymbol{a}}\right)^{2}\right)_{\boldsymbol{a} \in \mathfrak{K}_{\boldsymbol{c}}^{*}}, \\
\boldsymbol{Y}=\left(\theta_{\boldsymbol{a}}^{2} \delta\left(\psi_{\boldsymbol{a}+\boldsymbol{c}}+\psi_{\boldsymbol{a}}\right)\right)_{\boldsymbol{a} \in \mathfrak{K}_{\boldsymbol{c}}^{*}}
\end{gathered}
$$

Соотношения (5.13) в матричном виде дают

$$
\begin{aligned}
4 \boldsymbol{W}+4 \boldsymbol{X}+\boldsymbol{Y} & =4 \mathrm{M}(-2 \boldsymbol{W}+\boldsymbol{X}+\boldsymbol{Y}) \\
4 \boldsymbol{W}+\boldsymbol{X}+\boldsymbol{Y} & =\mathrm{M}(4 \boldsymbol{W}+\boldsymbol{X}+\boldsymbol{Y})
\end{aligned}
$$

Из (5.14) получаем

$$
\left(\mathrm{M}-\frac{1}{4} E\right) \boldsymbol{Y}=2\left(\mathrm{M}+\frac{1}{2} E\right) \boldsymbol{W}-(\mathrm{M}-E) \boldsymbol{X} .
$$


Пользуясь леммой 11 и следствием 1 из нее, находим

$$
\boldsymbol{Y}=\frac{8}{3}\left(\mathrm{M}+\frac{1}{2} E\right) \boldsymbol{W}+\frac{4}{3}(\mathrm{M}-E) \boldsymbol{X} .
$$

Поскольку

$$
\delta\left(\theta_{\boldsymbol{a}}^{2}\left(\psi_{\boldsymbol{a}+\boldsymbol{c}}+\psi_{\boldsymbol{a}}\right)\right)=2 \theta_{\boldsymbol{a}}^{2}\left(\psi_{\boldsymbol{a}+\boldsymbol{c}}+\psi_{\boldsymbol{a}}\right)^{2}+\theta_{\boldsymbol{a}}^{2} \delta\left(\psi_{\boldsymbol{a}+\boldsymbol{c}}+\psi_{\boldsymbol{a}}\right), \quad \boldsymbol{a} \in \mathfrak{K}_{\boldsymbol{c}}^{*},
$$

$\delta$-дифференцирование соотношений (5.11) приводит к матричному соотношению

$$
2 \boldsymbol{X}+\boldsymbol{Y}=\mathrm{M}(2 \boldsymbol{X}+\boldsymbol{Y})
$$

Вычитая его из (5.15), получаем

$$
4 \boldsymbol{W}-\boldsymbol{X}=\mathrm{M}(4 \boldsymbol{W}-\boldsymbol{X}),
$$

откуда

$$
\begin{aligned}
\left(\mathrm{M}+\frac{1}{2} E\right) \boldsymbol{W} & =\frac{1}{4} \mathrm{M}(4 \boldsymbol{W}-\boldsymbol{X})+\frac{1}{2} \boldsymbol{W}+\frac{1}{4} \mathrm{M} \boldsymbol{X}=\frac{1}{4}(4 \boldsymbol{W}-\boldsymbol{X})+\frac{1}{2} \boldsymbol{W}+\frac{1}{4} \mathrm{M} \boldsymbol{X} \\
& =\frac{3}{2} \boldsymbol{W}+\frac{1}{4}(\mathrm{M}-E) \boldsymbol{X} .
\end{aligned}
$$

Подставляя соотношение (5.18) в (5.16), находим

$$
\boldsymbol{Y}=4 \boldsymbol{W}+2(\mathrm{M}-E) \boldsymbol{X}
$$

что в точности отвечает системе (5.9). Теорема доказана полностью.

ЗАмечАниЕ. Конечно, систему дифференциальных уравнений (5.9) можно записать “персонально" для функций (0.16), пользуясь соотношениями (2.1). Но мы ограничимся персонализацией (0.17) теоремы 1.

ДокаЗАТЕЛЬство ТЕоремы ОХиямЫ. Покажем теперь, как в случае $g=2$ из теоремы 2 следует теорема Охиямы. Выберем произвольную характеристику $\boldsymbol{c} \in \mathbb{Z}^{4} / 2 \mathbb{Z}^{4}, \boldsymbol{c} \neq \mathbf{0}$. Отвечающее ей множество $\mathfrak{K}_{\boldsymbol{c}}^{*}$ состоит из трех элементов, которые мы обозначим через $\boldsymbol{a}_{1}, \boldsymbol{a}_{2}, \boldsymbol{a}_{3}$, причем порядок этих элементов (для удобства) выберем так, чтобы первая строка матрищы $\mathrm{M}=\mathrm{M}_{\boldsymbol{c}}$ состояла только из положительных чисел:

$$
M=\frac{1}{2}\left(\begin{array}{ccc}
1 & 1 & 1 \\
1 & 1 & -1 \\
1 & -1 & 1
\end{array}\right)
$$

Полагая

$$
\theta_{j}=\theta_{\boldsymbol{a}_{j}}, \quad S_{j}=\psi_{\boldsymbol{a}_{j}+\boldsymbol{c}}+\psi_{\boldsymbol{a}_{j}}, \quad P_{j}=\psi_{\boldsymbol{a}_{j}+\boldsymbol{c}} \psi_{\boldsymbol{a}_{j}}, \quad j=1,2,3,
$$

перепишем систему дифференциальных уравнений (5.9) (или (5.19)) в виде

$$
\begin{aligned}
& \theta_{1}^{2} \delta S_{1}=4 \theta_{1}^{2} P_{1}-\left(\theta_{1}^{1} S_{1}^{2}-\theta_{2}^{2} S_{2}^{2}-\theta_{3}^{2} S_{3}^{2}\right), \\
& \theta_{2}^{2} \delta S_{2}=4 \theta_{2}^{2} P_{2}+\left(\theta_{1}^{2} S_{1}^{2}-\theta_{2}^{2} S_{2}^{2}-\theta_{3}^{2} S_{3}^{2}\right), \\
& \theta_{3}^{2} \delta S_{3}=4 \theta_{3}^{2} P_{3}+\left(\theta_{1}^{2} S_{1}^{2}-\theta_{2}^{2} S_{2}^{2}-\theta_{3}^{2} S_{3}^{2}\right) .
\end{aligned}
$$


Соотношения $(5.10),(5.11)$ в нашем случае примут вид

$$
\theta_{1}^{2}-\theta_{2}^{2}-\theta_{3}^{2}=0, \quad \theta_{1}^{2} S_{1}-\theta_{2}^{2} S_{2}-\theta_{3}^{2} S_{3}=0
$$

откуда

$$
\frac{S_{2}-S_{3}}{\theta_{1}^{2}}=\frac{S_{1}-S_{3}}{\theta_{2}^{2}}=\frac{S_{2}-S_{1}}{\theta_{3}^{2}}
$$

и, следовательно,

$$
\begin{aligned}
\theta_{1}^{2} S_{1}^{2}-\theta_{2}^{2} S_{2}^{2}-\theta_{3}^{2} S_{3}^{2} & =\theta_{1}^{2}\left(S_{1}-S_{2}\right)\left(S_{1}-S_{3}\right) \\
& =-\theta_{2}^{2}\left(S_{2}-S_{1}\right)\left(S_{2}-S_{3}\right) \\
& =-\theta_{3}^{2}\left(S_{3}-S_{1}\right)\left(S_{3}-S_{2}\right)
\end{aligned}
$$

Подставляя соотношения (5.23) в уравнения (5.21) и производя сокращения на тэта-константы, получим систему

$$
\begin{aligned}
& \delta S_{1}=4 P_{1}-\left(S_{1}-S_{2}\right)\left(S_{1}-S_{3}\right) \\
& \delta S_{2}=4 P_{2}-\left(S_{2}-S_{1}\right)\left(S_{2}-S_{3}\right) \\
& \delta S_{3}=4 P_{3}-\left(S_{3}-S_{1}\right)\left(S_{3}-S_{2}\right)
\end{aligned}
$$

В наших обозначениях система Охиямы (0.21) получается из системы (5.24) сложением любых двух уравнений:

$$
\begin{aligned}
& \delta\left(S_{1}+S_{2}\right)=4 P_{1}+4 P_{2}-\left(S_{1}-S_{2}\right)^{2}, \\
& \delta\left(S_{1}+S_{3}\right)=4 P_{1}+4 P_{3}-\left(S_{1}-S_{3}\right)^{2}, \\
& \delta\left(S_{2}+S_{3}\right)=4 P_{2}+4 P_{3}-\left(S_{2}-S_{3}\right)^{2},
\end{aligned}
$$

поскольку каждые две из трех пар $\left\{\boldsymbol{a}_{1}, \boldsymbol{a}_{1}+\boldsymbol{c}\right\},\left\{\boldsymbol{a}_{2}, \boldsymbol{a}_{2}+\boldsymbol{c}\right\},\left\{\boldsymbol{a}_{3}, \boldsymbol{a}_{3}+\boldsymbol{c}\right\}$ образуют вершины параллелограмма.

Таким образом, при $g=2$ теорема Охиямы следует из теоремы 2. Отметим, что этот способ доказательства системы (0.21) отличается от оригинального [22], [23], которое мы приводим в следующем параграфе.

Что можно сказать про величину (5.22), которая является двумерным аналогом (1.9)? Обозначая ее через $\varkappa=\varkappa_{c}$ и дифференцируя первое из ее определений (5.22) с помощью системы (5.24), находим

$$
\delta \varkappa=\frac{\delta S_{2}-\delta S_{3}-2 S_{1}\left(S_{2}-S_{3}\right)}{\theta_{1}^{2}}=\frac{\left(4 P_{2}-S_{2}^{2}\right)-\left(4 P_{3}-S_{3}^{2}\right)}{\theta_{1}^{2}} .
$$

Производя аналогичные дифференцирования второй и третьей величины, приходим к выводу, что

$$
\delta \varkappa=\frac{\left(4 P_{1}-S_{1}^{2}\right)-\left(4 P_{3}-S_{3}^{2}\right)}{\theta_{2}^{2}}=\frac{\left(4 P_{2}-S_{2}^{2}\right)-\left(4 P_{1}-S_{1}^{2}\right)}{\theta_{3}^{2}} .
$$


То, что величины (5.25) равны, следует также, подобно выводу соотношений (5.22), из равенства

$$
\theta_{1}^{2}\left(4 P_{1}-S_{1}^{2}\right)-\theta_{2}^{2}\left(4 P_{2}-S_{2}^{2}\right)-\theta_{3}^{2}\left(4 P_{3}-S_{3}^{2}\right)=0
$$

которое мы получили в доказательстве теоремы 2 (см. (5.17)). Отметим, что

$$
4 P_{j}-S_{j}^{2}=-\left(\psi_{\boldsymbol{a}_{j}+\boldsymbol{c}}-\psi_{\boldsymbol{a}_{j}}\right)^{2}
$$

поэтому величина величина (5.25) ненулевая (в противном случае четыре тэта-константы были бы связаны соотношением $\vartheta_{1} \vartheta_{2}=$ const $\vartheta_{3} \vartheta_{4}$, что невозможно). Для $\delta$-дифференцирования величин (5.25) приходится выходить за пределы нашего "маленького мирка", состоящего из шести функций, в “большой мир" десяти функций (0.18). Не утомляя читателя длинными выкладками, приведем окончательный результат:

$$
\begin{aligned}
\delta^{2} \varkappa & =\frac{2\left(4 P_{2}-S_{2}^{2}\right)\left(\Sigma-2 S_{2}-2 S_{1}\right)-2\left(4 P_{3}-S_{3}^{2}\right)\left(\Sigma-2 S_{3}-2 S_{1}\right)}{\theta_{1}^{2}} \\
& =\frac{2\left(4 P_{1}-S_{1}^{2}\right)\left(\Sigma-2 S_{1}-2 S_{2}\right)-2\left(4 P_{3}-S_{3}^{2}\right)\left(\Sigma-2 S_{3}-2 S_{2}\right)}{\theta_{2}^{2}} \\
& =\frac{2\left(4 P_{2}-S_{2}^{2}\right)\left(\Sigma-2 S_{2}-2 S_{3}\right)-2\left(4 P_{1}-S_{1}^{2}\right)\left(\Sigma-2 S_{1}-2 S_{3}\right)}{\theta_{3}^{2}}
\end{aligned}
$$

где через $\Sigma$ мы обозначили сумму всех десяти (в случае $g=2$ ) функций $(0.18)$. Величина (5.26), вообще говоря, тоже отлична от нуля. Мы обрываем на этом процесс дифференцирования величины $\varkappa$ и возврашаемся к обобшению тождеств (0.8) для $g=2$ и $g=3$ в $\S 8$.

\section{§ 6. Вперед к новым дифференциальным уравнениям}

Для доказательства теорем 1 и 2 мы использовали далеко не все ресурсы соотношений Римана и в этом параграфе постараемся заполнить этот пробел: предметом нашего исследования станут тождества (3.2). Во избежание громоздкости возникающих вычислений нам понадобится схема, отработанная в $\S 3$ и 5 .

Суммирование в правой части соотношений (3.2) происходит только по характеристикам $\boldsymbol{b} \in \mathfrak{K}$ таким, что

$$
\boldsymbol{b}, \boldsymbol{b}+\boldsymbol{c}, \boldsymbol{b}+\boldsymbol{d} \in \mathfrak{K}^{*} .
$$

Зафиксируем теперь две характеристики

$$
\boldsymbol{c}, \boldsymbol{d} \in \mathfrak{K}, \quad \boldsymbol{c} \neq \boldsymbol{d}, \quad \boldsymbol{c} \neq \mathbf{0}, \quad \boldsymbol{d} \neq \mathbf{0},
$$

с дополнительным условием

$$
|\boldsymbol{c}, \boldsymbol{d}| \equiv 0(\bmod 2)
$$

Поскольку

$$
(-1)^{|\boldsymbol{b}+\boldsymbol{c}+\boldsymbol{d}|}=(-1)^{|\boldsymbol{c}, \boldsymbol{d}|}(-1)^{|\boldsymbol{b}|}(-1)^{|\boldsymbol{b}+\boldsymbol{c}|}(-1)^{|\boldsymbol{b}+\boldsymbol{d}|},
$$


из (6.1) и (6.3) следует, что $\boldsymbol{b}+\boldsymbol{c}+\boldsymbol{d} \in \mathfrak{K}^{*}$. Как и в $\S 5$, факторгруппу $\mathfrak{K}_{\boldsymbol{c}, \boldsymbol{d}}=\mathfrak{K} /\{\mathbf{0}, \boldsymbol{c}$, $\boldsymbol{d}, \boldsymbol{c}+\boldsymbol{d}\}$ (и ее подмножества) мы будем отождествлять с множеством в $\mathfrak{K}$, фиксируя по одному элементу из классов эквивалентности. Множество четных характеристик $\mathfrak{K}_{\boldsymbol{c}, \boldsymbol{d}}^{*} \subset \mathfrak{K}_{\boldsymbol{c}, \boldsymbol{d}}$ состоит из классов эквивалентности, для которых выполнено (6.1) (а значит, и $\boldsymbol{b}+\boldsymbol{c}+\boldsymbol{d} \in \mathfrak{K}^{*}$ ).

Полагая на этот раз

$$
\begin{gathered}
\theta_{\boldsymbol{a}}(\boldsymbol{z})=(-1)^{t}\left(\boldsymbol{c}^{\prime}+\boldsymbol{d}^{\prime}\right) \boldsymbol{a}^{\prime \prime} \vartheta_{\boldsymbol{a}+\boldsymbol{c}+\boldsymbol{d}}(\boldsymbol{z}) \vartheta_{\boldsymbol{a}+\boldsymbol{c}}(\boldsymbol{z}) \vartheta_{\boldsymbol{a}+\boldsymbol{d}}(\boldsymbol{z}) \vartheta_{\boldsymbol{a}}(\boldsymbol{z}), \\
\widetilde{\theta}_{\boldsymbol{a}}(\boldsymbol{z})=\theta_{\boldsymbol{a}}(\mathbf{0}) \cdot \frac{1}{4}\left(\frac{\vartheta_{\boldsymbol{a}+\boldsymbol{c}+\boldsymbol{d}}(2 \boldsymbol{z})}{\vartheta_{\boldsymbol{a}+\boldsymbol{c}+\boldsymbol{d}}(\mathbf{0})}+\frac{\vartheta_{\boldsymbol{a}+\boldsymbol{c}}(2 \boldsymbol{z})}{\vartheta_{\boldsymbol{a}+\boldsymbol{c}}(\mathbf{0})}+\frac{\vartheta_{\boldsymbol{a}+\boldsymbol{d}}(2 \boldsymbol{z})}{\vartheta_{\boldsymbol{a}+\boldsymbol{d}}(\mathbf{0})}+\frac{\vartheta_{\boldsymbol{a}}(2 \boldsymbol{z})}{\vartheta_{\boldsymbol{a}}(\mathbf{0})}\right), \\
\boldsymbol{a} \in \mathfrak{K}_{\boldsymbol{c}, \boldsymbol{d}}^{*},
\end{gathered}
$$

перепишем соотношения (3.2) в виде

$$
\theta_{\boldsymbol{a}}(\boldsymbol{z})=\frac{1}{2^{g-2}} \sum_{\boldsymbol{b} \in \mathfrak{K}_{\boldsymbol{c}, \boldsymbol{d}}^{*}}(-1)^{|\boldsymbol{a}, \boldsymbol{b}|} \widetilde{\theta}_{\boldsymbol{b}}(\boldsymbol{z}), \quad \boldsymbol{a} \in \mathfrak{K}_{\boldsymbol{c}, \boldsymbol{d}}^{*} .
$$

На самом деле, корректность формулы (6.4) (независимость суммирования в правой части от выбора элемента из класса эквивалентности в $\left.\mathfrak{K}_{\boldsymbol{c}, \boldsymbol{d}}\right)$ следует из следующего соотношения:

$$
\vartheta_{\boldsymbol{a}+2 \boldsymbol{e}}(\boldsymbol{z})=(-1)^{t} \boldsymbol{a}^{\prime} \boldsymbol{e}^{\prime \prime} \vartheta_{\boldsymbol{a}}(\boldsymbol{z}), \quad \boldsymbol{a}, \boldsymbol{e} \in \mathbb{Z}^{2 g}
$$

(см. $[1 ;$ ч. 2, гл. $7, \S 1])$. Поэтому несложная проверка показывает, что в случае выполнения условий $(6.1)$ и $(6.6)$ величина $(-1)^{|\boldsymbol{a}, \boldsymbol{b}|} \widetilde{\boldsymbol{\theta}}_{\boldsymbol{b}}(\boldsymbol{z})$ остается неизменной при замене $\boldsymbol{b}$ на $\boldsymbol{b}+\boldsymbol{c}, \boldsymbol{b}+\boldsymbol{d}$ или $\boldsymbol{b}+\boldsymbol{c}+\boldsymbol{d}$.

Точно так же, как это делалось при доказательстве лемм 8 и 11, устанавливается, что отвечающая тождествам (6.4) симметричная квадратная матрица

$$
\mathrm{M}=\mathrm{M}_{\boldsymbol{c}, \boldsymbol{d}}=\frac{1}{2^{g-2}}\left((-1)^{|\boldsymbol{a}, \boldsymbol{b}|}\right)_{\boldsymbol{a}, \boldsymbol{b} \in \mathfrak{K}_{\boldsymbol{c}, \boldsymbol{d}}^{*}}
$$

удовлетворяет равенству

$$
\mathrm{M}^{2}=\frac{1}{2}(\mathrm{M}+E)
$$

и количество элементов множества $\mathfrak{K}_{\boldsymbol{c}, \boldsymbol{d}}^{*}$ равно $2^{g-3}\left(2^{g-2}+1\right)$.

Для простоты записи положим

$$
\begin{aligned}
\theta_{\boldsymbol{a}} & =\theta_{\boldsymbol{a}}(\mathbf{0})=(-1)^{t}\left(\boldsymbol{c}^{\prime}+\boldsymbol{d}^{\prime}\right) \boldsymbol{a}^{\prime \prime} \vartheta_{\boldsymbol{a}+\boldsymbol{c}+\boldsymbol{d}} \vartheta_{\boldsymbol{a}+\boldsymbol{c}} \vartheta_{\boldsymbol{a}+\boldsymbol{d}} \vartheta_{\boldsymbol{a}}, \\
S_{\boldsymbol{a}} & =\psi_{\boldsymbol{a}+\boldsymbol{c}+\boldsymbol{d}}+\psi_{\boldsymbol{a}+\boldsymbol{c}}+\psi_{\boldsymbol{a}+\boldsymbol{d}}+\psi_{\boldsymbol{a}}, \\
P_{\boldsymbol{a}} & =\frac{1}{2}\left(S_{\boldsymbol{a}}^{2}-\psi_{\boldsymbol{a}+\boldsymbol{c}+\boldsymbol{d}}^{2}-\psi_{\boldsymbol{a}+\boldsymbol{c}}^{2}-\psi_{\boldsymbol{a}+\boldsymbol{d}}^{2}-\psi_{\boldsymbol{a}}^{2}\right),
\end{aligned}
$$

ТЕОРема 3. Пусть характеристики (6.2) удовлетворяют дополнительному условию (6.3) и $\mathfrak{K}_{\boldsymbol{c}, \boldsymbol{d}}^{*}$ - отвечающее им множество характеристик. Тогда в обозначениях (6.6) имеет место система дифференциальных уравнений

$$
\theta_{\boldsymbol{a}} \delta S_{\boldsymbol{a}}=\frac{1}{3 \cdot 2^{g-5}} \sum_{\boldsymbol{b} \in \mathfrak{K}_{\boldsymbol{c}, \boldsymbol{d}}^{*}}(-1)^{|\boldsymbol{a}, \boldsymbol{b}|} \theta_{\boldsymbol{b}} P_{\boldsymbol{b}}+\frac{4}{3} \theta_{\boldsymbol{a}} P_{\boldsymbol{a}}-\theta_{\boldsymbol{a}} S_{\boldsymbol{a}}^{2}, \quad \boldsymbol{a} \in \mathfrak{K}_{\boldsymbol{c}, \boldsymbol{d}}^{*},
$$


и выполнены соотночения

$$
\begin{aligned}
\theta_{\boldsymbol{a}} & =\frac{1}{2^{g-2}} \sum_{\boldsymbol{b} \in \mathfrak{K}_{\boldsymbol{c}, \boldsymbol{d}}^{*}}(-1)^{|\boldsymbol{a}, \boldsymbol{b}|} \theta_{\boldsymbol{b}}, \quad \boldsymbol{a} \in \mathfrak{K}_{\boldsymbol{c}, \boldsymbol{d}}^{*}, \\
\theta_{\boldsymbol{a}} S_{\boldsymbol{a}} & =\frac{1}{2^{g-2}} \sum_{\boldsymbol{b} \in \mathfrak{K}_{\boldsymbol{c}, \boldsymbol{d}}^{*}}(-1)^{|\boldsymbol{a}, \boldsymbol{b}|} \theta_{\boldsymbol{b}} S_{\boldsymbol{b}}, \quad \boldsymbol{a} \in \mathfrak{K}_{\boldsymbol{c}, \boldsymbol{d}}^{*} .
\end{aligned}
$$

ДокАЗАТЕЛьство. Согласно лемме 2 имеем

$$
\begin{aligned}
& \theta_{\boldsymbol{a}}(\boldsymbol{z})=\theta \cdot\left(1-2 S_{\boldsymbol{a}}+4 P_{\boldsymbol{a}}+\frac{2}{3}\left(S_{\boldsymbol{a}}^{2}-2 P_{\boldsymbol{a}}\right)+\frac{2}{3} \delta S_{\boldsymbol{a}}\right)+O\left(\boldsymbol{z}^{6}\right) \\
& \widetilde{\theta}_{\boldsymbol{a}}(\boldsymbol{z})=\theta \cdot\left(1-2 S_{\boldsymbol{a}}+\frac{8}{3}\left(S_{\boldsymbol{a}}^{2}-2 P_{\boldsymbol{a}}\right)+\frac{8}{3} \delta S_{\boldsymbol{a}}\right)+O\left(\boldsymbol{z}^{6}\right)
\end{aligned}
$$

Подставляя разложения (6.9) в тождества (6.4) и сравнивая коэффициенты при однородных по $\boldsymbol{z}$ компонентах степени 0,2 и 4, получаем соотношения (6.8) и

$$
\begin{aligned}
\theta_{\boldsymbol{a}}\left(\frac{8}{3} P_{\boldsymbol{a}}+\frac{2}{3} S_{\boldsymbol{a}}^{2}+\frac{2}{3} \delta S_{\boldsymbol{a}}\right)=\frac{1}{2^{g-2}} \sum_{\boldsymbol{b} \in \mathfrak{K}_{\boldsymbol{c}, \boldsymbol{d}}^{*}}(-1)^{|\boldsymbol{a}, \boldsymbol{b}|} \theta_{\boldsymbol{b}}\left(-\frac{16}{3} P_{\boldsymbol{b}}+\frac{8}{3} S_{\boldsymbol{b}}^{2}+\frac{8}{3} \delta S_{\boldsymbol{b}}\right) \\
\boldsymbol{a} \in \mathfrak{K}_{\boldsymbol{c}, \boldsymbol{d}}^{*}
\end{aligned}
$$

Полагая

$$
\boldsymbol{W}=\left(\theta_{\boldsymbol{a}} P_{\boldsymbol{a}}\right)_{\boldsymbol{a} \in \mathfrak{K}_{\boldsymbol{c}, \boldsymbol{d}}^{*}}, \quad \boldsymbol{X}=\left(\theta_{\boldsymbol{a}} S_{\boldsymbol{a}}^{2}\right)_{\boldsymbol{a} \in \mathfrak{K}_{\boldsymbol{c}, \boldsymbol{d}}^{*}}, \quad \boldsymbol{Y}=\left(\theta_{\boldsymbol{a}} \delta S_{\boldsymbol{a}}\right)_{\boldsymbol{a} \in \mathfrak{K}_{\boldsymbol{c}, \boldsymbol{d}}^{*}}
$$

и переписывая соотношения (6.10) в матричном виде:

$$
\frac{8}{3} \boldsymbol{W}+\frac{2}{3} \boldsymbol{X}+\frac{2}{3} \boldsymbol{Y}=-\frac{16}{3} \mathrm{M} \boldsymbol{W}+\frac{8}{3} \mathrm{M} \boldsymbol{X}+\frac{8}{3} \mathrm{M} \boldsymbol{Y}
$$

находим

$$
\left(\mathrm{M}-\frac{1}{4} E\right)(\boldsymbol{X}+\boldsymbol{Y})=2\left(\mathrm{M}+\frac{1}{2} E\right) \boldsymbol{W}
$$

откуда

$$
\boldsymbol{Y}=\frac{8}{3}\left(\mathrm{M}+\frac{1}{2} E\right) \boldsymbol{W}-\boldsymbol{X}
$$

Раскрытие матричного равенства (6.11) завершает доказательство теоремы.

"Недостатком" системы (6.7) является то обстоятельство, что под знаком суммы в правой части появляются суммы попарных произведений функций (0.18) (ср. с системами (0.20) и (5.9)). Именно этот факт не позволяет получить в случае $g=3$ систему без знаменателей подобно тому, как это было проделано при доказательстве теорем Альфена и Охиямы. К сожалению, использование других специализаций соотношений Римана дает лишш косметический эффект воздействия на систему (6.7), разрушая при этом ее внутреннюю симметрию. 
В случае $g=2$ количество элементов в множестве $\mathfrak{K}_{\boldsymbol{c}, \boldsymbol{d}}^{*}$ равно 1 и, значит, матрица М является числом: $\mathrm{M}=1$. Следовательно, все тэта-коэффициенты в уравнениях (6.7) сокращаются и мы получаем систему (0.21):

$$
\delta S_{\boldsymbol{a}}=4 P_{\boldsymbol{a}}-S_{\boldsymbol{a}}^{2}
$$

Тем самым, теорема Охиямы в очередной раз доказана.

Покажем теперь, как в случае $g=2$ из системы (0.21) получаются дифференциальные уравнения для каждой функции (0.18). Сопоставляя парам $(0,0),(0,1)$, $(1,0)$ и $(1,1)$ числа $0,1,2,3$ соответственно, мы будем записывать характеристику $\boldsymbol{a}=\left(\boldsymbol{a}^{\prime}, \boldsymbol{a}^{\prime \prime}\right) \in \mathbb{Z}^{4} / 2 \mathbb{Z}^{4}$ в виде двух чисел, первое из которых отвечает $\boldsymbol{a}^{\prime}$, а второе $\boldsymbol{a}^{\prime \prime}$.

В случае $g=2$

$$
\mathfrak{K}^{*}=\{00,01,02,03,10,12,20,21,30,33\} .
$$

Несложный подсчет показывает, что при разном выборе характеристик (6.2) различных уравнений вида (6.12) в точности пятнадщать, отвечающих наборам характеристик

$\{00,01,02,03\},\{00,01,20,21\},\{02,03,20,21\},\{00,02,10,12\},\{01,03,10,12\}$, $\{00,03,30,33\},\{01,02,30,33\},\{00,10,20,30\},\{02,12,20,30\},\{01,10,21,30\}$, $\{03,12,21,30\},\{00,12,21,33\},\{02,10,21,33\},\{01,12,20,33\},\{03,10,20,33\}$,

причем каждая из десяти тэта-констант попадает ровно в шесть из них. Суммируя все эти уравнения, находим

$$
\delta \sum_{\boldsymbol{a} \in \mathfrak{K}^{*}} \psi_{\boldsymbol{a}}=-\sum_{\boldsymbol{a} \in \mathfrak{K}^{*}} \psi_{\boldsymbol{a}}^{2}+\frac{1}{3} \sum_{\substack{\boldsymbol{a}, \boldsymbol{b} \in \mathfrak{K}^{*} \\ \boldsymbol{a} \neq \boldsymbol{b}}} \psi_{\boldsymbol{a}} \psi_{\boldsymbol{b}}
$$

Каждую характеристику $\boldsymbol{a}_{0} \in \mathfrak{K}^{*}$ можно собрать с оставшимися девятью четными в три “параллелограмма":

$$
a_{0}, a_{1}, a_{2}, a_{3}, \quad a_{0}, a_{4}, a_{5}, a_{6}, \quad a_{0}, a_{7}, a_{8}, a_{9}
$$

(отметим, что выбор таких четверок можно осуществить в точности двумя способами). Каждая из четверок (6.14) удовлетворяет уравнению (6.12), суммируя которые, находим

$$
\begin{aligned}
2 \delta \psi_{\boldsymbol{a}_{0}} & +\delta \sum_{\boldsymbol{a} \in \mathfrak{K}^{*}} \psi_{\boldsymbol{a}} \\
= & -2 \psi_{\boldsymbol{a}_{0}}^{2}-\sum_{\boldsymbol{a} \in \mathfrak{K}^{*}} \psi_{\boldsymbol{a}}^{2}+2 \psi_{\boldsymbol{a}_{0}} \sum_{\substack{\boldsymbol{a} \in \mathfrak{K}^{*} \\
\boldsymbol{a} \neq \boldsymbol{a}_{0}}} \psi_{\boldsymbol{a}}+2\left(\psi_{\boldsymbol{a}_{1}} \psi_{\boldsymbol{a}_{2}}+\psi_{\boldsymbol{a}_{1}} \psi_{\boldsymbol{a}_{3}}+\psi_{\boldsymbol{a}_{2}} \psi_{\boldsymbol{a}_{3}}\right) \\
& +2\left(\psi_{\boldsymbol{a}_{4}} \psi_{\boldsymbol{a}_{5}}+\psi_{\boldsymbol{a}_{4}} \psi_{\boldsymbol{a}_{6}}+\psi_{\boldsymbol{a}_{5}} \psi_{\boldsymbol{a}_{6}}\right)+2\left(\psi_{\boldsymbol{a}_{7}} \psi_{\boldsymbol{a}_{8}}+\psi_{\boldsymbol{a}_{7}} \psi_{\boldsymbol{a}_{9}}+\psi_{\boldsymbol{a}_{8}} \psi_{\boldsymbol{a}_{9}}\right) .
\end{aligned}
$$


Вычитая из (6.15) уравнение (6.13), получаем

$$
\begin{aligned}
\delta \psi_{\boldsymbol{a}_{0}}= & -\psi_{\boldsymbol{a}_{0}}^{2}+\psi_{\boldsymbol{a}_{0}} \sum_{\substack{\boldsymbol{a} \in \mathfrak{K}^{*} \\
\boldsymbol{a} \neq \boldsymbol{a}_{0}}} \psi_{\boldsymbol{a}}-\frac{1}{6} \sum_{\substack{\boldsymbol{a}, \boldsymbol{b} \in \mathfrak{K}^{*} \\
\boldsymbol{a} \neq \boldsymbol{b}}} \psi_{\boldsymbol{a}} \psi_{\boldsymbol{b}}+\left(\psi_{\boldsymbol{a}_{1}} \psi_{\boldsymbol{a}_{2}}+\psi_{\boldsymbol{a}_{1}} \psi_{\boldsymbol{a}_{3}}+\psi_{\boldsymbol{a}_{2}} \psi_{\boldsymbol{a}_{3}}\right) \\
& +\left(\psi_{\boldsymbol{a}_{4}} \psi_{\boldsymbol{a}_{5}}+\psi_{\boldsymbol{a}_{4}} \psi_{\boldsymbol{a}_{6}}+\psi_{\boldsymbol{a}_{5}} \psi_{\boldsymbol{a}_{6}}\right)+\left(\psi_{\boldsymbol{a}_{7}} \psi_{\boldsymbol{a}_{8}}+\psi_{\boldsymbol{a}_{7}} \psi_{\boldsymbol{a}_{9}}+\psi_{\boldsymbol{a}_{8}} \psi_{\boldsymbol{a}_{9}}\right) \\
= & -2 \psi_{\boldsymbol{a}_{0}}^{2}-\frac{1}{3}\left(\psi_{\boldsymbol{a}_{0}}^{2}+\psi_{\boldsymbol{a}_{1}}^{2}+\cdots+\psi_{\boldsymbol{a}_{9}}^{2}\right)-\frac{1}{6}\left(\psi_{\boldsymbol{a}_{0}}+\psi_{\boldsymbol{a}_{1}}+\cdots+\psi_{\boldsymbol{a}_{9}}\right)^{2} \\
& +\frac{1}{2}\left(\psi_{\boldsymbol{a}_{0}}+\psi_{\boldsymbol{a}_{1}}+\psi_{\boldsymbol{a}_{2}}+\psi_{\boldsymbol{a}_{3}}\right)^{2}+\frac{1}{2}\left(\psi_{\boldsymbol{a}_{0}}+\psi_{\boldsymbol{a}_{4}}+\psi_{\boldsymbol{a}_{5}}+\psi_{\boldsymbol{a}_{6}}\right)^{2} \\
& +\frac{1}{2}\left(\psi_{\boldsymbol{a}_{0}}+\psi_{\boldsymbol{a}_{7}}+\psi_{\boldsymbol{a}_{8}}+\psi_{\boldsymbol{a}_{9}}\right)^{2} .
\end{aligned}
$$

Для завершения "персонализации” системы уравнений Охиямы, приведем все разбиения на четверки (6.14) для каждой четной характеристики $\boldsymbol{a}_{0}$ :

\begin{tabular}{|c|l|l|l|}
\hline $\boldsymbol{a}_{0}$ & $\left\{\boldsymbol{a}_{0}, \boldsymbol{a}_{1}, \boldsymbol{a}_{2}, \boldsymbol{a}_{3}\right\}$ & $\left\{\boldsymbol{a}_{0}, \boldsymbol{a}_{4}, \boldsymbol{a}_{5}, \boldsymbol{a}_{6}\right\}$ & $\left\{\boldsymbol{a}_{0}, \boldsymbol{a}_{7}, \boldsymbol{a}_{8}, \boldsymbol{a}_{9}\right\}$ \\
\hline 00 & $\{00,01,02,03\}$ & $\{00,10,20,30\}$ & $\{00,12,21,33\}$ \\
& $\{00,01,20,21\}$ & $\{00,02,10,12\}$ & $\{00,03,30,33\}$ \\
\hline 01 & $\{00,01,02,03\}$ & $\{01,10,21,30\}$ & $\{01,12,20,33\}$ \\
& $\{00,01,20,21\}$ & $\{01,03,10,12\}$ & $\{01,02,30,33\}$ \\
\hline 02 & $\{00,01,02,03\}$ & $\{02,12,20,30\}$ & $\{02,10,21,33\}$ \\
& $\{02,03,20,21\}$ & $\{00,02,10,12\}$ & $\{01,02,30,33\}$ \\
\hline 03 & $\{00,01,02,03\}$ & $\{03,12,21,30\}$ & $\{03,10,20,33\}$ \\
& $\{02,03,20,21\}$ & $\{01,03,10,12\}$ & $\{00,03,30,33\}$ \\
\hline 10 & $\{00,02,10,12\}$ & $\{01,10,21,30\}$ & $\{03,10,20,33\}$ \\
& $\{01,03,10,12\}$ & $\{00,10,20,30\}$ & $\{02,10,21,33\}$ \\
\hline 12 & $\{00,02,10,12\}$ & $\{03,12,21,30\}$ & $\{01,12,20,33\}$ \\
& $\{01,03,10,12\}$ & $\{02,12,20,30\}$ & $\{00,12,21,33\}$ \\
\hline 20 & $\{00,01,20,21\}$ & $\{02,12,20,30\}$ & $\{03,10,20,33\}$ \\
& $\{02,03,20,21\}$ & $\{00,10,20,30\}$ & $\{01,12,20,33\}$ \\
\hline 21 & $\{00,01,20,21\}$ & $\{03,12,21,30\}$ & $\{02,10,21,33\}$ \\
& $\{02,03,20,21\}$ & $\{01,10,21,30\}$ & $\{00,12,21,33\}$ \\
\hline 30 & $\{00,03,30,33\}$ & $\{02,12,20,30\}$ & $\{01,10,21,30\}$ \\
& $\{01,02,30,33\}$ & $\{00,10,20,30\}$ & $\{03,12,21,30\}$ \\
\hline 33 & $\{00,03,30,33\}$ & $\{02,10,21,33\}$ & $\{01,12,20,33\}$ \\
& $\{01,02,30,33\}$ & $\{00,12,21,33\}$ & $\{03,10,20,33\}$ \\
\hline
\end{tabular}

Каждое из разбиений (6.14) на четверки определяет уравнение (6.16), и для любой четной характеристики $\boldsymbol{a}_{0}$ выбор разбиений осушествляется двумя способами. Поэтому уравнения (6.16) дают десять квадратичных соотношений вида

$$
\begin{aligned}
& \left(\psi_{\boldsymbol{a}_{1}}+\psi_{\boldsymbol{a}_{2}}+\psi_{\boldsymbol{a}_{3}}\right)^{2}+\left(\psi_{\boldsymbol{a}_{4}}+\psi_{\boldsymbol{a}_{5}}+\psi_{\boldsymbol{a}_{6}}\right)^{2}+\left(\psi_{\boldsymbol{a}_{7}}+\psi_{\boldsymbol{a}_{8}}+\psi_{\boldsymbol{a}_{9}}\right)^{2} \\
& \quad=\left(\psi_{\boldsymbol{a}_{1}^{*}}+\psi_{\boldsymbol{a}_{2}^{*}}+\psi_{\boldsymbol{a}_{3}^{*}}\right)^{2}+\left(\psi_{\boldsymbol{a}_{4}^{*}}+\psi_{\boldsymbol{a}_{5}^{*}}+\psi_{\boldsymbol{a}_{6}^{*}}\right)^{2}+\left(\psi_{\boldsymbol{a}_{7}^{*}}+\psi_{\boldsymbol{a}_{8}^{*}}+\psi_{\boldsymbol{a}_{9}^{*}}\right)^{2}
\end{aligned}
$$


(звездочкой отмечены элементы второго разбиения). Как показывают вычисления (см. также [22; предложение 4.1]), при $g=2$ идеал $I$ в колье $\mathbb{Q}\left[x_{\boldsymbol{a}}\right]_{\boldsymbol{a} \in \mathfrak{K}^{*}}$, порожденный (в соответствии с указанной вьше таблицей) десятью однородными многочленами второй степени

$$
\begin{aligned}
& y_{\boldsymbol{a}_{0}}=\left(x_{\boldsymbol{a}_{1}}+x_{\boldsymbol{a}_{2}}+x_{\boldsymbol{a}_{3}}\right)^{2}+\left(x_{\boldsymbol{a}_{4}}+x_{\boldsymbol{a}_{5}}+x_{\boldsymbol{a}_{6}}\right)^{2}+\left(x_{\boldsymbol{a}_{7}}+x_{\boldsymbol{a}_{8}}+x_{\boldsymbol{a}_{9}}\right)^{2} \\
& \quad-\left(x_{\boldsymbol{a}_{1}^{*}}+x_{\boldsymbol{a}_{2}^{*}}+x_{\boldsymbol{a}_{3}^{*}}\right)^{2}-\left(x_{\boldsymbol{a}_{4}^{*}}+x_{\boldsymbol{a}_{5}^{*}}+x_{\boldsymbol{a}_{6}^{*}}\right)^{2}-\left(x_{\boldsymbol{a}_{7}^{*}}+x_{\boldsymbol{a}_{8}^{*}}+x_{\boldsymbol{a}_{9}^{*}}\right)^{2}, \quad \boldsymbol{a}_{0} \in \mathfrak{K}^{*},
\end{aligned}
$$

имеет размерность 6 .

На самом деле, система (6.16) с использованием двух разбиений на параллелограммы может быть переписана в следуюшей компактной форме:

$$
\delta \psi_{\boldsymbol{a}}=-2 \psi_{\boldsymbol{a}}^{2}-\frac{1}{3} \sum_{\boldsymbol{b} \in \mathfrak{K}^{*}} \psi_{\boldsymbol{b}}^{2}-\frac{1}{6}\left(\sum_{\boldsymbol{b} \in \mathfrak{K}^{*}} \psi_{\boldsymbol{b}}\right)^{2}+\frac{1}{4} \sum_{\mathfrak{G} \ni \boldsymbol{a}}\left(\sum_{\boldsymbol{b} \in \mathfrak{G}} \psi_{\boldsymbol{b}}\right)^{2}, \quad \boldsymbol{a} \in \mathfrak{K}^{*},
$$

где суммирование $\sum_{\mathfrak{G} \ni \boldsymbol{a}}$ осушествляется по всем (шести) параллелограммам, содержашим характеристику $\boldsymbol{a} \in \mathfrak{K}^{*}$.

Tеорема 4. Пусть $g=3$ и заданной паре характеристик (6.2) с условием (6.3) отвечает множество $\mathfrak{K}_{\boldsymbol{c}, \boldsymbol{d}}^{*}=\left\{\boldsymbol{a}_{1}, \boldsymbol{a}_{2}, \boldsymbol{a}_{3}\right\}$. Тогда в обозначениях (6.6) имеет место система дифференциальных уравнений

$$
\begin{aligned}
& \delta S_{\boldsymbol{a}_{1}}=4 P_{\boldsymbol{a}_{1}}-S_{\boldsymbol{a}_{1}}^{2}-\frac{4}{3} \frac{\left(S_{\boldsymbol{a}_{2}}-S_{\boldsymbol{a}_{3}}\right) P_{\boldsymbol{a}_{1}}+\left(S_{\boldsymbol{a}_{3}}-S_{\boldsymbol{a}_{1}}\right) P_{\boldsymbol{a}_{2}}+\left(S_{\boldsymbol{a}_{1}}-S_{\boldsymbol{a}_{2}}\right) P_{\boldsymbol{a}_{3}}}{S_{\boldsymbol{a}_{2}}-S_{\boldsymbol{a}_{3}}}, \\
& \delta S_{\boldsymbol{a}_{2}}=4 P_{\boldsymbol{a}_{2}}-S_{\boldsymbol{a}_{2}}^{2}-\frac{4}{3} \frac{\left(S_{\boldsymbol{a}_{2}}-S_{\boldsymbol{a}_{3}}\right) P_{\boldsymbol{a}_{1}}+\left(S_{\boldsymbol{a}_{3}}-S_{\boldsymbol{a}_{1}}\right) P_{\boldsymbol{a}_{2}}+\left(S_{\boldsymbol{a}_{1}}-S_{\boldsymbol{a}_{2}}\right) P_{\boldsymbol{a}_{3}}}{S_{\boldsymbol{a}_{3}}-S_{\boldsymbol{a}_{1}}}, \\
& \delta S_{\boldsymbol{a}_{3}}=4 P_{\boldsymbol{a}_{3}}-S_{\boldsymbol{a}_{3}}^{2}-\frac{4}{3} \frac{\left(S_{\boldsymbol{a}_{2}}-S_{\boldsymbol{a}_{3}}\right) P_{\boldsymbol{a}_{1}}+\left(S_{\boldsymbol{a}_{3}}-S_{\boldsymbol{a}_{1}}\right) P_{\boldsymbol{a}_{2}}+\left(S_{\boldsymbol{a}_{1}}-S_{\boldsymbol{a}_{2}}\right) P_{\boldsymbol{a}_{3}}}{S_{\boldsymbol{a}_{1}}-S_{\boldsymbol{a}_{2}}} .
\end{aligned}
$$

ДокАЗАТЕЛЬСТво. Несложньй подсчет показывает, что в случае $g=3$ множество $\mathfrak{K}_{\boldsymbol{c}, \boldsymbol{d}}^{*}$ трехэлементно. Не ограничивая общности, порядок элементов $\boldsymbol{a}_{1}, \boldsymbol{a}_{2}$, $\boldsymbol{a}_{3}$ считаем выбранным так, что матрица $\mathrm{M}=\mathrm{M}_{\boldsymbol{c}, \boldsymbol{d}}$ имеет вид (5.20). Согласно теореме 3 мы получаем систему дифференциальных уравнений

$$
\begin{aligned}
& \theta_{\boldsymbol{a}_{1}} \delta S_{\boldsymbol{a}_{1}}=4 \theta_{\boldsymbol{a}_{1}} P_{\boldsymbol{a}_{1}}-\theta_{\boldsymbol{a}_{1}} S_{\boldsymbol{a}_{1}}^{2}-\frac{4}{3}\left(\theta_{\boldsymbol{a}_{1}} P_{\boldsymbol{a}_{1}}-\theta_{\boldsymbol{a}_{2}} P_{\boldsymbol{a}_{2}}-\theta_{\boldsymbol{a}_{3}} P_{\boldsymbol{a}_{3}}\right), \\
& \theta_{\boldsymbol{a}_{2}} \delta S_{\boldsymbol{a}_{2}}=4 \theta_{\boldsymbol{a}_{2}} P_{\boldsymbol{a}_{2}}-\theta_{\boldsymbol{a}_{2}} S_{\boldsymbol{a}_{2}}^{2}+\frac{4}{3}\left(\theta_{\boldsymbol{a}_{1}} P_{\boldsymbol{a}_{1}}-\theta_{\boldsymbol{a}_{2}} P_{\boldsymbol{a}_{2}}-\theta_{\boldsymbol{a}_{3}} P_{\boldsymbol{a}_{3}}\right), \\
& \theta_{\boldsymbol{a}_{3}} \delta S_{\boldsymbol{a}_{3}}=4 \theta_{\boldsymbol{a}_{3}} P_{\boldsymbol{a}_{3}}-\theta_{\boldsymbol{a}_{3}} S_{\boldsymbol{a}_{3}}^{2}+\frac{4}{3}\left(\theta_{\boldsymbol{a}_{1}} P_{\boldsymbol{a}_{1}}-\theta_{\boldsymbol{a}_{2}} P_{\boldsymbol{a}_{2}}-\theta_{\boldsymbol{a}_{3}} P_{\boldsymbol{a}_{3}}\right) ;
\end{aligned}
$$

соотношения (6.8) преобразуются в нашем случае к виду

$$
\frac{S_{\boldsymbol{a}_{2}}-S_{\boldsymbol{a}_{3}}}{\theta_{\boldsymbol{a}_{1}}}=\frac{S_{\boldsymbol{a}_{1}}-S_{\boldsymbol{a}_{3}}}{\theta_{\boldsymbol{a}_{2}}}=\frac{S_{\boldsymbol{a}_{2}}-S_{\boldsymbol{a}_{1}}}{\theta_{\boldsymbol{a}_{3}}}
$$


Из соотношений (6.20) следует, что

$$
\begin{aligned}
\theta_{\boldsymbol{a}_{1}} & P_{\boldsymbol{a}_{1}}-\theta_{\boldsymbol{a}_{2}} P_{\boldsymbol{a}_{2}}-\theta_{\boldsymbol{a}_{3}} P_{\boldsymbol{a}_{3}} \\
& =\theta_{\boldsymbol{a}_{1}} \frac{\left(S_{\boldsymbol{a}_{2}}-S_{\boldsymbol{a}_{3}}\right) P_{\boldsymbol{a}_{1}}+\left(S_{\boldsymbol{a}_{3}}-S_{\boldsymbol{a}_{1}}\right) P_{\boldsymbol{a}_{2}}+\left(S_{\boldsymbol{a}_{1}}-S_{\boldsymbol{a}_{2}}\right) P_{\boldsymbol{a}_{3}}}{S_{\boldsymbol{a}_{2}}-S_{\boldsymbol{a}_{3}}} \\
& =-\theta_{\boldsymbol{a}_{2}} \frac{\left(S_{\boldsymbol{a}_{2}}-S_{\boldsymbol{a}_{3}}\right) P_{\boldsymbol{a}_{1}}+\left(S_{\boldsymbol{a}_{3}}-S_{\boldsymbol{a}_{1}}\right) P_{\boldsymbol{a}_{2}}+\left(S_{\boldsymbol{a}_{1}}-S_{\boldsymbol{a}_{2}}\right) P_{\boldsymbol{a}_{3}}}{S_{\boldsymbol{a}_{3}}-S_{\boldsymbol{a}_{1}}} \\
& =-\theta_{\boldsymbol{a}_{3}} \frac{\left(S_{\boldsymbol{a}_{2}}-S_{\boldsymbol{a}_{3}}\right) P_{\boldsymbol{a}_{1}}+\left(S_{\boldsymbol{a}_{3}}-S_{\boldsymbol{a}_{1}}\right) P_{\boldsymbol{a}_{2}}+\left(S_{\boldsymbol{a}_{1}}-S_{\boldsymbol{a}_{2}}\right) P_{\boldsymbol{a}_{3}}}{S_{\boldsymbol{a}_{1}}-S_{\boldsymbol{a}_{2}}} .
\end{aligned}
$$

Деля обе части уравнений (6.19) на соответстующие тэта-константы, с помощью соотношений (6.21) получаем систему дифференциальных уравнений (6.18). Теорема доказана.

Не останавливаясь подробно на дифференциальных уравнениях для каждой функции (0.18) в случае $g=3$, укажем способ их получения из систем (6.18). Фиксируя пару четных характеристик $\boldsymbol{a}_{1}, \boldsymbol{a}_{2}$ и отвечающую им характеристику $\boldsymbol{c}=\boldsymbol{a}_{2}-\boldsymbol{a}_{1}$ в паре (6.2), выберем четные характеристики $\boldsymbol{a}_{3}, \boldsymbol{a}_{4}=\boldsymbol{a}_{3}+\boldsymbol{c}$, $\boldsymbol{a}_{5}, \boldsymbol{a}_{6}=\boldsymbol{a}_{5}+\boldsymbol{c}$. Каждая из четверок

$$
\left\{a_{1}, a_{2}, a_{3}, a_{4}\right\}, \quad\left\{a_{1}, a_{2}, a_{5}, a_{6}\right\}, \quad\left\{a_{3}, a_{4}, a_{5}, a_{6}\right\}
$$

образует вершины параллелограмма, так что система (6.18) позволяет выписать дифференциальные уравнения для величин

$\delta\left(\psi \boldsymbol{a}_{1}+\psi \boldsymbol{a}_{2}+\psi_{\boldsymbol{a}_{3}}+\psi_{\boldsymbol{a}_{4}}\right), \quad \delta\left(\psi_{\boldsymbol{a}_{1}}+\psi_{\boldsymbol{a}_{2}}+\psi_{\boldsymbol{a}_{5}}+\psi_{\boldsymbol{a}_{6}}\right), \quad \delta\left(\psi_{\boldsymbol{a}_{3}}+\psi_{\boldsymbol{a}_{4}}+\psi_{\boldsymbol{a}_{5}}+\psi_{\boldsymbol{a}_{6}}\right)$,

из которых получаем дифференциальные уравнения для $\delta\left(\psi_{\boldsymbol{a}_{1}}+\psi_{\boldsymbol{a}_{2}}\right)$ и $\delta\left(\psi_{\boldsymbol{a}_{3}}+\psi_{\boldsymbol{a}_{4}}\right)$ со степенью знаменателя равной 3 . В частности, мы получаем уравнение для

$$
\delta\left(2 \psi_{\boldsymbol{a}_{1}}+2 \psi_{\boldsymbol{a}_{2}}-\psi_{\boldsymbol{a}_{3}}-\psi_{\boldsymbol{a}_{4}}\right)
$$

с тем же знаменателем в правой части. Аналогично определяются уравнения для

$$
\delta\left(2 \psi_{\boldsymbol{a}_{1}}+2 \psi_{\boldsymbol{a}_{3}}-\psi_{\boldsymbol{a}_{2}}-\psi_{\boldsymbol{a}_{4}}\right), \quad \delta\left(2 \psi_{\boldsymbol{a}_{1}}+2 \psi_{\boldsymbol{a}_{4}}-\psi_{\boldsymbol{a}_{2}}-\psi_{\boldsymbol{a}_{3}}\right),
$$

каждое из которых имеет свой знаменатель степени 3. Сложение получившихся дифференциальных уравнений для величин (6.22), (6.23) дает уравнение для $\delta \psi_{\boldsymbol{a}_{1}}$ со степенью знаменателя 9 в правой части. Разумеется, в силу свободы выбора характеристик $\boldsymbol{a}_{2}, \boldsymbol{a}_{3}, \boldsymbol{a}_{5}, \ldots$ дифференциальные уравнения для функции $\psi_{\boldsymbol{a}_{1}}$ получаются различные, значит возникает большое количество алгебраических соотношений между функциями (0.18). Но напомним, что в случае $g=3$ имеется 36 четных характеристик, поэтому комбинаторную задачу выявления всех возникающих алгебраических связей мы оставляем за пределами этой работы. 


\section{§7. Системы характеристик и тэта-соотношения}

В этом параграфе мы проведем ревизию встречавшихся ранее специализаций соотношений Римана (3.1). Прежде всего, мы легализуем аддитивные системы характеристик, частный случай которых в размерности $g=2$ мы называли параллелограммом.

Характеристики $\boldsymbol{a}, \boldsymbol{b} \in \mathfrak{K}=\mathbb{Z}^{2 g} / 2 \mathbb{Z}^{2 g}$ называются сизигетичнымми, если число $|\boldsymbol{a}, \boldsymbol{b}|$ четно. Для двух сизигетичных характеристик определим величину $\boldsymbol{a} \boldsymbol{b}={ }^{t} \boldsymbol{a}^{\prime} \boldsymbol{b}^{\prime \prime}(\bmod 2)$; поскольку $\boldsymbol{a} \boldsymbol{b}+\boldsymbol{b} \boldsymbol{a} \equiv|\boldsymbol{a}, \boldsymbol{b}|(\bmod 2)$, имеем $\boldsymbol{a} \boldsymbol{b}=\boldsymbol{b} \boldsymbol{a}$. Кроме того, $\boldsymbol{a} \boldsymbol{a}=|\boldsymbol{a}|$.

Под аддитивной группой характеристик $\mathfrak{A} \subset \mathfrak{K}$ мы понимаем группу по сложению в $\mathfrak{K}$, элементы которой попарно сизигетичны. В этой группе можно выбрать $\boldsymbol{l}$ базисных векторов $\boldsymbol{a}_{1}, \ldots, \boldsymbol{a}_{\boldsymbol{l}}$ (не единственным образом) так, что $\mathfrak{A}=\left\{\alpha_{1} \boldsymbol{a}_{1}+\cdots\right.$ $\left.+\alpha_{l} \boldsymbol{a}_{l}: \alpha_{1}, \ldots, \alpha_{l}=0,1\right\} ;$ при этом $(-1)^{\left|\boldsymbol{a}_{j}, \boldsymbol{a}_{k}\right|}=1$ для всех $j, k=1, \ldots, l$. Число элементов в аддитивной групше $\mathfrak{A}$ равно $2^{l}$, и мы называем $l$ размерностью, полагая $\operatorname{dim} \mathfrak{A}=l$. Сразу отметим, что $\operatorname{dim} \mathfrak{A} \leqslant g($ см. [1; ч. 2, гл. $7, \S 8])$. Определенная выше коммутативная операция умножения сизигетичных характеристик дистрибутивна относительно сложения в аддитивной группе $\mathfrak{A}$.

Аддитивная система характеристик $\mathfrak{G}$ - это множество четных характеристик, всевозможные попарные суммы которых образуют аддитивную группу; такую систему можно представить в виде $\mathfrak{G}=\boldsymbol{a}+\mathfrak{A} \subset \mathfrak{K}^{*}$, где $\boldsymbol{a}-$ произвольный элемент $\mathfrak{G}$, а $\mathfrak{A}$ - соответствующая (однозначно определенная) аддитивная группа. Под размерностью аддитивной системы мы будем понимать размерность соответствующей аддитивной группы $(\operatorname{dim} \mathfrak{G}=\operatorname{dim} \mathfrak{A})$, количество элементов при этом равно $2^{\operatorname{dim} \mathfrak{G}}$. Каждая четная характеристика является аддитивной системой размерности 0 . Аддитивная система максимальной размерности $g$ назьвается гёпелевой системой; примеры таких систем в размерности $g=2$ появлялись в связи с теоремой Охиямы.

ЛЕмма 12. Количество аддитивных систем в $\mathbb{Z}^{2 g} / 2 \mathbb{Z}^{2 g}$ размерности $l \leqslant g$ равно

$$
2^{g-l-1}\left(2^{g-l}+1\right) \cdot \frac{\left(2^{2 g}-1\right)\left(2^{2 g-2}-1\right) \cdots\left(2^{2(g-l+1)}-1\right)}{\left(2^{l}-1\right)\left(2^{l-1}-1\right) \cdots(2-1)} .
$$

В частности, количество гёпелевых систем равно

$$
\frac{\left(2^{2 g}-1\right)\left(2^{2 g-2}-1\right) \cdots\left(2^{2}-1\right)}{\left(2^{g}-1\right)\left(2^{g-1}-1\right) \cdots(2-1)}=\left(2^{g}+1\right)\left(2^{g-1}+1\right) \cdots(2+1) .
$$

ДокАЗАТЕльство. На самом деле, данная лемма является чисто комбинаторным фактом: количество аддитивных групп характеристик размерности $l$ в $\mathfrak{K}$ равHO

$$
\frac{\left(2^{2 g}-1\right)\left(2^{2 g-2}-1\right) \cdots\left(2^{2(g-l+1)}-1\right)}{\left(2^{l}-1\right)\left(2^{l-1}-1\right) \cdots(2-1)}
$$

(см. [1; ч. 2, гл. $7, \S 8])$. Для каждой такой группы $\mathfrak{A}$ факторгруппа $\mathfrak{K} / \mathfrak{A}$ изоморфна $\mathbb{Z}^{2 g-2 l} / 2 \mathbb{Z}^{2 g-2 l}$, поэтому количество соответствующих аддитивных систем равно количеству четных характеристик в последней, именно $2^{g-l-1}\left(2^{g-l}+1\right)$. Тем самьм, лемма доказана. 
СлЕДСТВИЕ. Количество аддитивных систем в $\mathbb{Z}^{2 g} / 2 \mathbb{Z}^{2 g}$ размерности $l \leqslant g$, содержсащих данную четную характеристику а, равно

$$
\left(2^{g-l}+1\right)\left(2^{g}-1\right) \cdot \frac{\left(2^{2 g-2}-1\right) \cdots\left(2^{2(g-l+1)}-1\right)}{\left(2^{l}-1\right)\left(2^{l-1}-1\right) \cdots(2-1)} .
$$

ДокАзАТЕЛЬство. Прежде всего, отметим, что количество $\varkappa_{\boldsymbol{a}}$ аддитивных систем из формулировки не зависит от выбора $\boldsymbol{a} \in \mathfrak{K}^{*}$. Поскольку количество элементов в $\mathfrak{K}^{*}$ равно $2^{g-1}\left(2^{g}+1\right)$ и в каждой аддитивной системе принимает участие $2^{l}$ характеристик, имеем

$$
\varkappa_{\boldsymbol{a}}=\frac{2^{l} \cdot \varkappa}{2^{g-1}\left(2^{g}+1\right)}=\frac{\varkappa}{2^{g-l-1}\left(2^{g}+1\right)}, \quad a \in \mathfrak{K}^{*},
$$

где $\varkappa$ - общее количество аддитивных систем размерности $l$. Пользуясь теперь точным выражением для $\varkappa$ из леммы 12 , получаем необходимое утверждение.

Все следуюшие рассуждения имеют смысл только при $g \geqslant 3$. Зафиксируем ненулевой элемент $\boldsymbol{c} \in \mathfrak{K}$, а значит, множество $\mathfrak{K}_{\boldsymbol{c}}^{*}=\mathfrak{K} /\{\boldsymbol{0}, \boldsymbol{c}\}$. Возврашаясь к обозначениям (5.3) из $\S 5$, перепишем соотношения (3.2) (или (6.4)) в виде

$$
\begin{gathered}
\theta_{\boldsymbol{a}+\boldsymbol{d}}(\boldsymbol{z}) \theta_{\boldsymbol{a}}(\boldsymbol{z})=\frac{1}{2^{g-2}} \sum_{\boldsymbol{b} \in \mathfrak{K}_{c}^{*}}(-1)^{|\boldsymbol{a}, \boldsymbol{b}|+{ }^{t} \boldsymbol{d}^{\prime}\left(\boldsymbol{a}^{\prime \prime}+\boldsymbol{b}^{\prime \prime}\right)} \frac{1}{2}\left(\widetilde{\theta}_{\boldsymbol{b}+\boldsymbol{d}}(\boldsymbol{z}) \theta_{\boldsymbol{b}}+\theta_{\boldsymbol{b}+\boldsymbol{d}} \widetilde{\theta}_{\boldsymbol{b}}(\boldsymbol{z})\right), \\
\boldsymbol{a} \in \mathfrak{K}_{\boldsymbol{c}}^{*}, \quad \boldsymbol{d} \in \mathfrak{D},
\end{gathered}
$$

где $\mathfrak{D}=\mathfrak{D}_{\boldsymbol{c}}=\mathfrak{K}_{\boldsymbol{c}}^{*}+\boldsymbol{a} \subset \mathfrak{K}_{\boldsymbol{c}}$ для некоторого (любого) $\boldsymbol{a} \in \mathfrak{K}_{\boldsymbol{c}}^{*}$.

Преобразуем (7.1) к виду

$$
\begin{aligned}
\theta_{\boldsymbol{a}+\boldsymbol{d}}(\boldsymbol{z}) \theta_{\boldsymbol{a}}(\boldsymbol{z})-\frac{1}{2^{g-1}}\left(\widetilde{\theta}_{\boldsymbol{a}+\boldsymbol{d}}(\boldsymbol{z}) \theta_{\boldsymbol{a}}+\theta_{\boldsymbol{a}+\boldsymbol{d}} \widetilde{\theta}_{\boldsymbol{a}}(\boldsymbol{z})\right) \\
\quad=\frac{1}{2^{g-2}} \sum_{\substack{\boldsymbol{b} \in \mathfrak{K}_{\boldsymbol{c}}^{*} \\
\boldsymbol{b} \neq \boldsymbol{a}, \boldsymbol{a}+\boldsymbol{d}}}(-1)^{|\boldsymbol{a}, \boldsymbol{b}|+{ }^{t} \boldsymbol{d}^{\prime}\left(\boldsymbol{a}^{\prime \prime}+\boldsymbol{b}^{\prime \prime}\right)} \frac{1}{2}\left(\widetilde{\theta}_{\boldsymbol{b}+\boldsymbol{d}}(\boldsymbol{z}) \theta_{\boldsymbol{b}}+\theta_{\boldsymbol{b}+\boldsymbol{d}} \widetilde{\theta}_{\boldsymbol{b}}(\boldsymbol{z})\right),
\end{aligned}
$$

$$
a \in \mathfrak{K}_{c}^{*}, \quad d \in \mathfrak{D},
$$

домножим обе части соотношений $(7.2)$ на $(-1)^{|\boldsymbol{a}, \boldsymbol{d}|} \theta_{\boldsymbol{a}+\boldsymbol{d}}$ и просуммируем по $\boldsymbol{d} \in$ $\mathfrak{D} \backslash\{\mathbf{0}\}$, пользуясь (только в левой части) формулами $(5.4),(5.5)$ :

$$
\begin{aligned}
& 2\left(2^{g-2}-1\right)\left(\theta_{\boldsymbol{a}}^{2}(\boldsymbol{z}) \theta_{\boldsymbol{a}}-\frac{1}{2^{g-1}} \widetilde{\theta}_{\boldsymbol{a}}(\boldsymbol{z}) \theta_{\boldsymbol{a}}^{2}\right) \\
& =\frac{1}{2^{g-2}} \sum_{\mathfrak{G}=\left\{\boldsymbol{a}, \boldsymbol{a}_{1}, \boldsymbol{a}_{2}, \boldsymbol{a}_{3}\right\}}(-1)^{|\mathfrak{G}|}\left(\widetilde{\theta}_{\boldsymbol{a}_{1}}(\boldsymbol{z}) \theta_{\boldsymbol{a}_{2}} \theta_{\boldsymbol{a}_{3}}+\theta_{\boldsymbol{a}_{1}} \widetilde{\theta}_{\boldsymbol{a}_{2}}(\boldsymbol{z}) \theta_{\boldsymbol{a}_{3}}+\theta_{\boldsymbol{a}_{1}} \theta_{\boldsymbol{a}_{2}} \widetilde{\theta}_{\boldsymbol{a}_{3}}(\boldsymbol{z})\right), \\
& \boldsymbol{a} \in \mathfrak{K}_{\boldsymbol{c}}^{*},
\end{aligned}
$$


где суммирование в правой части происходит по всем аддитивным системам $\mathfrak{G}=$ $\{\boldsymbol{a}, \boldsymbol{a}+\boldsymbol{d}, \boldsymbol{a}+\boldsymbol{e}, \boldsymbol{a}+\boldsymbol{d}+\boldsymbol{e}\}$ в $\mathfrak{K}_{\boldsymbol{c}}^{*}$ размерности 2, а величина

$$
|\mathfrak{G}|=|\boldsymbol{d}|+|\boldsymbol{e}|+\boldsymbol{d e}(\bmod 2)
$$

не зависит от выбора элемента $\boldsymbol{a} \in \mathfrak{G}$ и образуюших $\boldsymbol{d}, \boldsymbol{e}$ соответствуюшей аддитивной группы $\mathfrak{G}+\boldsymbol{a} \subset \mathfrak{K}_{\boldsymbol{c}}$.

Подстановка $\boldsymbol{z}=\mathbf{0}$ в (7.3) дает

$$
\frac{1}{3} \cdot \frac{2\left(2^{g-2}-1\right)\left(2^{g-1}-1\right)}{2^{g-1}} \theta_{\boldsymbol{a}}^{3}=\frac{1}{2^{g-2}} \sum_{\mathfrak{G}=\left\{\boldsymbol{a}, \boldsymbol{a}_{1}, \boldsymbol{a}_{2}, \boldsymbol{a}_{3}\right\}}(-1)^{|\mathfrak{G}|} \theta_{\boldsymbol{a}_{1}} \theta_{\boldsymbol{a}_{2}} \theta_{\boldsymbol{a}_{3}}, \quad \boldsymbol{a} \in \mathfrak{K}_{\boldsymbol{c}}^{*} .
$$

Умножая обе части (7.3) на $\theta_{\boldsymbol{a}}$, обе части (7.4) на $\widetilde{\theta}_{\boldsymbol{a}}(\boldsymbol{z})$ и складывая получающиеся соотношения, находим

$$
2\left(2^{g-2}-1\right)\left(\theta_{\boldsymbol{a}}^{2}(\boldsymbol{z}) \theta_{\boldsymbol{a}}^{2}+\frac{1}{3}\left(1-\frac{1}{2^{g-3}}\right) \widetilde{\theta}_{\boldsymbol{a}}(\boldsymbol{z}) \theta_{\boldsymbol{a}}^{3}\right)=\frac{1}{2^{g-2}} \sum_{\mathfrak{G} \ni \boldsymbol{a}} \widetilde{\theta}_{\mathfrak{G}}(\boldsymbol{z}), \quad \boldsymbol{a} \in \mathfrak{K}_{\boldsymbol{c}}^{*}
$$

где

$$
\begin{gathered}
\widetilde{\theta}_{\mathfrak{G}}(\boldsymbol{z})=(-1)^{|\mathfrak{G}|} \theta_{\boldsymbol{a}} \theta_{\boldsymbol{a}_{1}} \theta_{\boldsymbol{a}_{2}} \theta_{\boldsymbol{a}_{3}} \cdot\left(\frac{\widetilde{\theta}_{\boldsymbol{a}}(\boldsymbol{z})}{\theta_{\boldsymbol{a}}}+\frac{\widetilde{\theta}_{\boldsymbol{a}_{1}}(\boldsymbol{z})}{\theta_{\boldsymbol{a}_{1}}}+\frac{\widetilde{\theta}_{\boldsymbol{a}_{2}}(\boldsymbol{z})}{\theta_{\boldsymbol{a}_{2}}}+\frac{\widetilde{\theta}_{\boldsymbol{a}_{3}}(\boldsymbol{z})}{\theta_{\boldsymbol{a}_{3}}}\right), \\
\mathfrak{G}=\left\{\boldsymbol{a}, \boldsymbol{a}_{1}, \boldsymbol{a}_{2}, \boldsymbol{a}_{3}\right\} \ni \boldsymbol{a} .
\end{gathered}
$$

Вспоминая теперь наши определения (5.3) и переходя от двумерных аддитивных систем $\mathfrak{G}$ в $\mathfrak{K}_{\boldsymbol{c}}$ к трехмерньп системам $\mathfrak{F}=\{\boldsymbol{a}, \boldsymbol{a}+\boldsymbol{c}: \boldsymbol{a} \in \mathfrak{G}\}$ в $\mathfrak{K}$, перепишем соотношения (7.5):

$$
\begin{aligned}
& \left(2^{g-2}-1\right)(-1)^{|\boldsymbol{a}, \boldsymbol{c}|} \vartheta_{\boldsymbol{a}+\boldsymbol{c}}^{2}(\boldsymbol{z}) \vartheta_{\boldsymbol{a}+\boldsymbol{c}}^{2} \vartheta_{\boldsymbol{a}}^{2}(\boldsymbol{z}) \vartheta_{\boldsymbol{a}}^{2} \\
& \quad+\frac{\left(2^{g-3}-1\right)\left(2^{g-2}-1\right)}{3 \cdot 2^{g-2}}(-1)^{|\boldsymbol{a}, \boldsymbol{c}|}\left(\vartheta_{\boldsymbol{a}+\boldsymbol{c}}(2 \boldsymbol{z}) \vartheta_{\boldsymbol{a}}+\vartheta_{\boldsymbol{a}+\boldsymbol{c}} \vartheta_{\boldsymbol{a}}(2 \boldsymbol{z})\right) \vartheta_{\boldsymbol{a}+\boldsymbol{c}}^{3} \vartheta_{\boldsymbol{a}}^{3} \\
& \quad=\frac{1}{2^{g-3}} \sum_{\mathfrak{F} \supset\{\boldsymbol{a}, \boldsymbol{a}+\boldsymbol{c}\}} \vartheta_{\mathfrak{F}}(2 \boldsymbol{z}), \quad \boldsymbol{a} \in \mathfrak{K}^{*}, \quad \boldsymbol{c} \neq \mathbf{0},
\end{aligned}
$$

где суммирование в правой части происходит по всем аддитивным системам $\mathfrak{F}=$ $\{\boldsymbol{a}, \boldsymbol{a}+\boldsymbol{c}, \boldsymbol{a}+\boldsymbol{d}, \boldsymbol{a}+\boldsymbol{e}, \boldsymbol{a}+\boldsymbol{c}+\boldsymbol{d}, \boldsymbol{a}+\boldsymbol{c}+\boldsymbol{e}, \boldsymbol{a}+\boldsymbol{d}+\boldsymbol{e}, \boldsymbol{a}+\boldsymbol{c}+\boldsymbol{d}+\boldsymbol{e}\}$ в $\mathfrak{K}$ и

$$
\vartheta_{\mathfrak{F}}(\boldsymbol{z})=(-1)^{|\boldsymbol{c}|+|\boldsymbol{d}|+|\boldsymbol{e}|+\boldsymbol{c d}+\boldsymbol{d e}+\boldsymbol{e c}} \prod_{\boldsymbol{b} \in \mathfrak{F}} \vartheta_{\boldsymbol{b}} \cdot \frac{1}{8} \sum_{\boldsymbol{b} \in \mathfrak{F}} \frac{\vartheta_{\boldsymbol{b}}(\boldsymbol{z})}{\vartheta_{\boldsymbol{b}}} .
$$

ЛЕмма 13. Величина

$$
\begin{gathered}
\vartheta_{\mathfrak{F}}=\vartheta_{\mathfrak{F}}(\mathbf{0})=(-1)^{|\boldsymbol{c}|+|\boldsymbol{d}|+|\boldsymbol{e}|+\boldsymbol{c d}+\boldsymbol{d e}+\boldsymbol{e} \boldsymbol{c}} \vartheta_{\boldsymbol{a}} \vartheta_{\boldsymbol{a}+\boldsymbol{c}} \vartheta_{\boldsymbol{a}+\boldsymbol{d}} \vartheta_{\boldsymbol{a}+\boldsymbol{e}} \\
\times \vartheta_{\boldsymbol{a}+\boldsymbol{c}+\boldsymbol{d}} \vartheta_{\boldsymbol{a}+\boldsymbol{c}+\boldsymbol{e} \vartheta_{\boldsymbol{a}+\boldsymbol{d}+\boldsymbol{e}} \vartheta_{\boldsymbol{a}+\boldsymbol{c}+\boldsymbol{d}+\boldsymbol{e}}}
\end{gathered}
$$

не зависит от выбора әлемента а аддитивной системы $\mathfrak{F}$ и образующих $\boldsymbol{c}, \boldsymbol{d}, \boldsymbol{e}$ соответствующей аддитивной әруппъъ $\mathfrak{F}+\boldsymbol{a}$. 
ДоКАЗАТЕЛЬСТво. Ввиду симметрии величины (7.8) относительно выбора образуюших $\boldsymbol{c}, \boldsymbol{d}, \boldsymbol{e}$ аддитивной группы $\mathfrak{F}+\boldsymbol{a}$ достаточно проверить инвариантность $\vartheta_{\mathfrak{F}}$ при заменах $\boldsymbol{a}=\boldsymbol{b}+\boldsymbol{c}$ на $\boldsymbol{b}$ (независимость от выбора элемента аддитивной системы $\mathfrak{F}$ ) и $\boldsymbol{c}=\boldsymbol{b}+\boldsymbol{d}$ на $\boldsymbol{b}$ (независимость от выбора образующих аддитивной группы $\mathfrak{F}+\boldsymbol{a})$. Справедливость этих утверждений доказывается непосредственньми вычислениями, при этом используется формула (6.5). Детали вычислений мы оставляем читателю.

Из леммы 13 следует корректность определенной нами величины (7.7). Таким образом, мы доказали следующее утверждение.

ЛЕмма 14. Справедливь следующие соотношения:

$$
\begin{aligned}
\left(2^{g-2}-1\right)(-1)^{|\boldsymbol{a}, \boldsymbol{b}|} \vartheta_{\boldsymbol{a}}^{2}(\boldsymbol{z}) \vartheta_{\boldsymbol{a}}^{2} \vartheta_{\boldsymbol{b}}^{2}(\boldsymbol{z}) \vartheta_{\boldsymbol{b}}^{2} & \\
& +\frac{\left(2^{g-3}-1\right)\left(2^{g-2}-1\right)}{3 \cdot 2^{g-2}}(-1)^{|\boldsymbol{a}, \boldsymbol{b}|}\left(\vartheta_{\boldsymbol{a}}(2 \boldsymbol{z}) \vartheta_{\boldsymbol{b}}+\vartheta_{\boldsymbol{a}} \vartheta_{\boldsymbol{b}}(2 \boldsymbol{z})\right) \vartheta_{\boldsymbol{a}}^{3} \vartheta_{\boldsymbol{b}}^{3} \\
& =\frac{1}{2^{g-3}} \sum_{\mathfrak{F} \supset\{\boldsymbol{a}, \boldsymbol{b}\}} \vartheta_{\mathfrak{F}}(2 \boldsymbol{z}), \quad \boldsymbol{a}, \boldsymbol{b} \in \mathfrak{K}^{*}, \quad \boldsymbol{a} \neq \boldsymbol{b},
\end{aligned}
$$

где суммирование в правой части происходит по всем аддитивным системам $\mathfrak{F} \supset\{\boldsymbol{a}, \boldsymbol{b}\}$ в $\mathfrak{K}$ размерности 3, а функиии $\vartheta_{\mathfrak{F}}(\boldsymbol{z})$ определяются равенствами (7.7). В частности, при $g=3$ справедливы соотношения

$$
(-1)^{|\boldsymbol{a}, \boldsymbol{b}|} \vartheta_{\boldsymbol{a}}^{2}(\boldsymbol{z}) \vartheta_{\boldsymbol{a}}^{2} \vartheta_{\boldsymbol{b}}^{2}(\boldsymbol{z}) \vartheta_{\boldsymbol{b}}^{2}=\sum_{\mathfrak{F} \supset\{\boldsymbol{a}, \boldsymbol{b}\}} \vartheta_{\mathfrak{F}}(2 \boldsymbol{z}), \quad \boldsymbol{a}, \boldsymbol{b} \in \mathfrak{K}^{*}, \quad \boldsymbol{a} \neq \boldsymbol{b} .
$$

ДокАЗАтЕЛЬство. Для доказательства достаточно положить $\boldsymbol{b}=\boldsymbol{a}+\boldsymbol{c}$ B (7.6).

Займемся суммированием (7.9) по всем $\boldsymbol{b} \in \mathfrak{K}^{*} \backslash\{\boldsymbol{a}\}$. Для левых частей мы воспользуемся следствиями из соотношений Римана (3.1):

$$
\begin{aligned}
& \sum_{\substack{\boldsymbol{b} \in \mathfrak{K}^{*} \\
\boldsymbol{b} \neq \boldsymbol{a}}}(-1)^{|\boldsymbol{a}, \boldsymbol{b}|} \vartheta_{\boldsymbol{b}}^{2}(\boldsymbol{z}) \vartheta_{\boldsymbol{b}}^{2}=\left(2^{g}-1\right) \vartheta_{\boldsymbol{a}}^{2}(\boldsymbol{z}) \vartheta_{\boldsymbol{a}}^{2}, \quad \boldsymbol{a} \in \mathfrak{K}^{*}, \\
& \sum_{\substack{\boldsymbol{b} \in \mathfrak{K}^{*} \\
\boldsymbol{b} \neq \boldsymbol{a}}}(-1)^{|\boldsymbol{a}, \boldsymbol{b}|} \vartheta_{\boldsymbol{b}}^{4}=\left(2^{g}-1\right) \vartheta_{\boldsymbol{a}}^{4}, \quad \boldsymbol{a} \in \mathfrak{K}^{*}, \\
& \sum_{\substack{\boldsymbol{b} \in \mathfrak{K}^{*} \\
\boldsymbol{b} \neq \boldsymbol{a}}}(-1)^{|\boldsymbol{a}, \boldsymbol{b}|} \vartheta_{\boldsymbol{b}}(2 \boldsymbol{z}) \vartheta_{\boldsymbol{b}}^{3}=2^{g} \vartheta_{\boldsymbol{a}}^{4}(\boldsymbol{z})-\vartheta_{\boldsymbol{a}}(2 \boldsymbol{z}) \vartheta_{\boldsymbol{a}}^{3}, \quad \boldsymbol{a} \in \mathfrak{K}^{*}
\end{aligned}
$$

Следовательно,

$$
\begin{aligned}
& \sum_{\substack{\boldsymbol{b} \in \mathfrak{K}^{*} \\
\boldsymbol{b} \neq \boldsymbol{a}}}\left(\left(2^{g-2}-1\right)(-1)^{|\boldsymbol{a}, \boldsymbol{b}|} \vartheta_{\boldsymbol{a}}^{2}(\boldsymbol{z}) \vartheta_{\boldsymbol{a}}^{2} \vartheta_{\boldsymbol{b}}^{2}(\boldsymbol{z}) \vartheta_{\boldsymbol{b}}^{2}\right. \\
& \left.\quad+\frac{\left(2^{g-2}-1\right)\left(2^{g-3}-1\right)}{3 \cdot 2^{g-2}}(-1)^{|\boldsymbol{a}, \boldsymbol{b}|}\left(\vartheta_{\boldsymbol{a}}(2 \boldsymbol{z}) \vartheta_{\boldsymbol{b}}+\vartheta_{\boldsymbol{a}} \vartheta_{\boldsymbol{b}}(2 \boldsymbol{z})\right) \vartheta_{\boldsymbol{a}}^{3} \vartheta_{\boldsymbol{b}}^{3}\right) \\
& =\frac{7\left(2^{g-2}-1\right)\left(2^{g-1}-1\right)}{3} \vartheta_{\boldsymbol{a}}^{4}(\boldsymbol{z}) \vartheta_{\boldsymbol{a}}^{4}+\frac{\left(2^{g-3}-1\right)\left(2^{g-2}-1\right)\left(2^{g-1}-1\right)}{3 \cdot 2^{g-3}} \vartheta_{\boldsymbol{a}}(2 \boldsymbol{z}) \vartheta_{\boldsymbol{a}}^{7}, \\
& \boldsymbol{a} \in \mathfrak{K}^{*} .
\end{aligned}
$$


Количество аддитивных систем $\mathfrak{F} \supset\{\boldsymbol{a}, \boldsymbol{b}\}$ в $\mathfrak{K}=\mathbb{Z}^{2 g} / 2 \mathbb{Z}^{2 g}$ размерности 3 равно количеству аддитивных систем $\mathfrak{G} \ni \boldsymbol{a}$ в $\mathfrak{K}_{\boldsymbol{b}-\boldsymbol{a}} \simeq \mathbb{Z}^{2 g-2} / 2 \mathbb{Z}^{2 g-2}$ размерности 2 , которое согласно следствию из леммы 12 равно

$$
\left(2^{g-3}+1\right)\left(2^{g-1}-1\right) \frac{2^{2 g-4}-1}{\left(2^{2}-1\right)(2-1)} .
$$

Обшее количество элементов множества $\mathfrak{K}^{*} \backslash\{\boldsymbol{a}\}$ равно

$$
2^{g-1}\left(2^{g}+1\right)-1=\left(2^{g-1}+1\right)\left(2^{g}-1\right) .
$$

Поэтому после суммирования правой части (7.9) по $\boldsymbol{b} \in \mathfrak{K}^{*} \backslash\{\boldsymbol{a}\}$ мы получим

$$
\begin{aligned}
\varkappa_{1} & =\left(2^{g-3}+1\right)\left(2^{g-1}+1\right)\left(2^{g-1}-1\right)\left(2^{g}-1\right) \frac{2^{2 g-4}-1}{\left(2^{2}-1\right)(2-1)} \\
& =\left(2^{g-3}+1\right)\left(2^{g}-1\right) \frac{\left(2^{2 g-2}-1\right)\left(2^{2 g-4}-1\right)}{\left(2^{2}-1\right)(2-1)}
\end{aligned}
$$

слагаемых, каждое из которых отвечает некоторой аддитивной системе $\mathfrak{F} \ni \boldsymbol{a}$ в $\mathfrak{K}$ размерности 3 . Количество таких аддитивных систем согласно следствию из леммы 12 равно

$$
\varkappa_{2}=\left(2^{g-3}+1\right)\left(2^{g}-1\right) \frac{\left(2^{2 g-2}-1\right)\left(2^{2 g-4}-1\right)}{\left(2^{3}-1\right)\left(2^{2}-1\right)(2-1)} .
$$

Следовательно,

$\frac{1}{2^{g-3}} \sum_{\substack{\boldsymbol{b} \in \mathfrak{K}^{*} \\ \boldsymbol{b} \neq \boldsymbol{a}}} \sum_{\mathfrak{F} \supset\{\boldsymbol{a}, \boldsymbol{b}\}} \vartheta_{\mathfrak{F}}(2 \boldsymbol{z})=\frac{1}{2^{g-3}} \cdot \frac{\varkappa_{1}}{\varkappa_{2}} \sum_{\mathfrak{F} \ni \boldsymbol{a}} \vartheta_{\mathfrak{F}}(2 \boldsymbol{z})=\frac{7}{2^{g-3}} \sum_{\mathfrak{F} \ni \boldsymbol{a}} \vartheta_{\mathfrak{F}}(2 \boldsymbol{z}), \quad \boldsymbol{a} \in \mathfrak{K}^{*}$.

Собирая вместе результаты суммирований (7.10) и (7.11), получаем следуюшее утверждение.

ЛЕмма 15. Справедливы следующие соотношения:

$$
\begin{aligned}
& \frac{\left(2^{g-2}-1\right)\left(2^{g-1}-1\right)}{3} \vartheta_{\boldsymbol{a}}^{4}(\boldsymbol{z}) \vartheta_{\boldsymbol{a}}^{4}+\frac{\left(2^{g-3}-1\right)\left(2^{g-2}-1\right)\left(2^{g-1}-1\right)}{3 \cdot 7 \cdot 2^{g-3}} \vartheta_{\boldsymbol{a}}(2 \boldsymbol{z}) \vartheta_{\boldsymbol{a}}^{7} \\
& =\frac{1}{2^{g-3}} \sum_{\mathfrak{F} \ni \boldsymbol{a}} \vartheta_{\mathfrak{F}}(2 \boldsymbol{z}), \quad \boldsymbol{a} \in \mathfrak{K}^{*}
\end{aligned}
$$

где суммирование в правой части происходит по всем аддитивным системам $\mathfrak{F} \ni \boldsymbol{a}$ в $\mathfrak{K}$ размерности 3 , а функиии $\vartheta_{\mathfrak{F}}(\boldsymbol{z})$ определяются равенствами (7.7). В частности, при $g=3$ справедливь соотношения

$$
\vartheta_{\boldsymbol{a}}^{4}(\boldsymbol{z}) \vartheta_{\boldsymbol{a}}^{4}=\sum_{\mathfrak{F} \ni \boldsymbol{a}} \vartheta_{\mathfrak{F}}(2 \boldsymbol{z}), \quad \boldsymbol{a} \in \mathfrak{K}^{*}
$$

Для аддитивной системы $\mathfrak{F}$ в $\mathfrak{K}, \operatorname{dim} \mathfrak{F}=3$, через $S_{\mathfrak{F}}$ и $P_{\mathfrak{F}}$ обозначим сумму функций $\psi_{\boldsymbol{b}}, \boldsymbol{b} \in \mathfrak{F}$, и сумму их всевозможных различных попарных произведений. Соотношения (7.12) позволяют получить новую систему дифференциальных уравнений для функций (0.18). 
ТЕОРема 5. Функиии (0.18) удовлетворяют системе дифференциальных уравнений

$\vartheta_{\boldsymbol{a}}^{8} \delta \psi_{\boldsymbol{a}}=-\frac{2}{3}\left(13-\frac{1}{2^{g-4}}\right) \vartheta_{\boldsymbol{a}}^{8} \psi_{\boldsymbol{a}}^{2}+\frac{1}{2^{g-3}\left(2^{g-2}-1\right)\left(2^{g-1}-1\right)} \sum_{\mathfrak{F} \ni \boldsymbol{a}} \vartheta_{\mathfrak{F}} P_{\mathfrak{F}}, \quad \boldsymbol{a} \in \mathfrak{K}^{*}$,

где суммирование в правой части происходит по всем аддитивным системам $\mathfrak{F} \ni \boldsymbol{a}$ в $\mathfrak{K}$ размерности 3 , функции $\vartheta_{\mathfrak{F}}$ определяются равенствами (7.8). Кроме того, справедливы соотношения

$$
\begin{aligned}
\vartheta_{\boldsymbol{a}}^{8} & =\frac{21}{\left(2^{g-2}-1\right)\left(2^{g-1}-1\right)\left(2^{g}-1\right)} \sum_{\mathfrak{F} \ni \boldsymbol{a}} \vartheta_{\mathfrak{F}}, \quad \boldsymbol{a} \in \mathfrak{K}^{*}, \\
\vartheta_{\boldsymbol{a}}^{8} \psi_{\boldsymbol{a}} & =\frac{21}{8\left(2^{g-2}-1\right)\left(2^{g-1}-1\right)\left(2^{g}-1\right)} \sum_{\mathfrak{F} \ni \boldsymbol{a}} \vartheta_{\mathfrak{F}} S_{\mathfrak{F}}, \quad \boldsymbol{a} \in \mathfrak{K}^{*} .
\end{aligned}
$$

ДоКАЗАТЕЛЬСТво. Для каждой аддитивной системы $\mathfrak{F}$ в $\mathfrak{K}$ размерности 3 положим

$$
\left(S_{\mathfrak{F}}\right)^{2}=\left(\sum_{\boldsymbol{a} \in \mathfrak{F}} \psi_{\boldsymbol{a}}\right)^{2}, \quad S_{\mathfrak{F}}^{2}=\sum_{\boldsymbol{a} \in \mathfrak{F}} \psi_{\boldsymbol{a}}^{2}
$$

и воспользуемся в обеих частях соотношений (7.12) разложениями (4.1) до $O\left(\boldsymbol{z}^{6}\right)$ :

$$
\begin{aligned}
& \frac{\left(2^{g-2}-1\right)\left(2^{g-1}-1\right)}{3} \vartheta_{\boldsymbol{a}}^{8} \cdot\left(1-8 \psi_{\boldsymbol{a}}+\frac{80}{3} \psi_{\boldsymbol{a}}^{2}+\frac{8}{3} \delta \psi_{\boldsymbol{a}}\right) \\
& \quad+\frac{\left(2^{g-3}-1\right)\left(2^{g-2}-1\right)\left(2^{g-1}-1\right)}{3 \cdot 7 \cdot 2^{g-3}} \vartheta_{\boldsymbol{a}}^{8} \cdot\left(1-8 \psi_{\boldsymbol{a}}+\frac{32}{3} \psi_{\boldsymbol{a}}^{2}+\frac{32}{3} \delta \psi_{\boldsymbol{a}}\right) \\
& =\frac{1}{2^{g-3}} \sum_{\mathfrak{F} \ni \boldsymbol{a}} \vartheta_{\mathfrak{F}} \cdot\left(1-S_{\mathfrak{F}}+\frac{4}{3} S_{\mathfrak{F}}^{2}+\frac{4}{3} \delta S_{\mathfrak{F}}\right), \quad \boldsymbol{a} \in \mathfrak{K}^{*},
\end{aligned}
$$

откуда

$$
\begin{gathered}
\frac{\left(2^{g-2}-1\right)\left(2^{g-1}-1\right)\left(2^{g}-1\right)}{3 \cdot 7} \vartheta_{\boldsymbol{a}}^{8}=\sum_{\mathfrak{F} \ni \boldsymbol{a}} \vartheta_{\mathfrak{F}}, \quad \boldsymbol{a} \in \mathfrak{K}^{*}, \\
\frac{\left(2^{g-2}-1\right)\left(2^{g-1}-1\right)\left(2^{g}-1\right)}{3 \cdot 7} \vartheta_{\boldsymbol{a}}^{8} \psi_{\boldsymbol{a}}=\frac{1}{8} \sum_{\mathfrak{F} \ni \boldsymbol{a}} \vartheta_{\mathfrak{F}} S_{\mathfrak{F}}, \quad \boldsymbol{a} \in \mathfrak{K}^{*}, \\
\frac{\left(2^{g-3}-1\right)\left(2^{g-2}-1\right)\left(2^{g-1}-1\right)}{3 \cdot 7} \vartheta_{\boldsymbol{a}}^{8}\left(74 \psi_{\boldsymbol{a}}^{2}+11 \delta \psi_{\boldsymbol{a}}\right) \\
+\frac{\left(2^{g-2}-1\right)\left(2^{g-1}-1\right)}{3} \vartheta_{\boldsymbol{a}}^{8}\left(10 \psi_{\boldsymbol{a}}^{2}+\delta \psi_{\boldsymbol{a}}\right)=\frac{1}{2} \sum_{\mathfrak{F} \ni \boldsymbol{a}} \vartheta_{\mathfrak{F}}\left(S_{\mathfrak{F}}^{2}+\delta S_{\mathfrak{F}}\right), \quad \boldsymbol{a} \in \mathfrak{K}^{*} .
\end{gathered}
$$

Соотношения (7.15), (7.16) приводят к (7.14); $\delta$-дифференцируя соотношения (7.16), получаем

$$
\frac{\left(2^{g-2}-1\right)\left(2^{g-1}-1\right)\left(2^{g}-1\right)}{3 \cdot 7} \vartheta_{\boldsymbol{a}}^{8}\left(8 \psi_{\boldsymbol{a}}^{2}+\delta \psi_{\boldsymbol{a}}\right)=\frac{1}{8} \sum_{\mathfrak{F} \ni \boldsymbol{a}} \vartheta_{\mathfrak{F}}\left(\left(S_{\mathfrak{F}}\right)^{2}+\delta S_{\mathfrak{F}}\right), \quad \boldsymbol{a} \in \mathfrak{K}^{*} .
$$


Умножая (7.18) на 4 и вычитая (7.17), находим

$$
\begin{aligned}
& \frac{2}{3}\left(13 \cdot 2^{g-3}-2\right)\left(2^{g-2}-1\right)\left(2^{g-1}-1\right) \vartheta_{\boldsymbol{a}}^{8} \psi_{\boldsymbol{a}}^{2}+2^{g-3}\left(2^{g-2}-1\right)\left(2^{g-1}-1\right) \vartheta_{\boldsymbol{a}}^{8} \delta \psi_{\boldsymbol{a}} \\
& \quad=\frac{1}{2} \sum_{\mathfrak{F} \ni \boldsymbol{a}} \vartheta_{\mathfrak{F}}\left(\left(S_{\mathfrak{F}}\right)^{2}-S_{\mathfrak{F}}^{2}\right), \quad \boldsymbol{a} \in \mathfrak{K}^{*}
\end{aligned}
$$

откуда следует система дифференциальных уравнений (7.13).

СледСТВИЕ. При $g=3$ функиии (0.18) удовлетворяют системе дифференииальных уравнений

$$
\vartheta_{\boldsymbol{a}}^{8} \delta \psi_{\boldsymbol{a}}=-\frac{22}{3} \vartheta_{\boldsymbol{a}}^{8} \psi_{\boldsymbol{a}}^{2}+\frac{1}{3} \sum_{\mathfrak{F} \ni \boldsymbol{a}} \vartheta_{\mathfrak{F}} P_{\mathfrak{F}}, \quad \boldsymbol{a} \in \mathfrak{K}^{*},
$$

где суммирование в правой части происходит по всем гёпелевым системам $\mathfrak{F} \ni \boldsymbol{a}$, функиии $\vartheta_{\mathfrak{F}}$ определяются равенствами (7.8) и $P_{\mathfrak{F}}-$ сумма всевозможсных попарных произведений функиий $\psi_{\boldsymbol{b}}, \boldsymbol{b} \in \mathfrak{F}$. Кроме того, справедливы соотношения

$$
\vartheta_{\boldsymbol{a}}^{8}=\sum_{\mathfrak{F} \ni \boldsymbol{a}} \vartheta_{\mathfrak{F}}, \quad \vartheta_{\boldsymbol{a}}^{8} \psi_{\boldsymbol{a}}=\frac{1}{8} \sum_{\mathfrak{F} \ni \boldsymbol{a}} \vartheta_{\mathfrak{F}} S_{\mathfrak{F}}, \quad \boldsymbol{a} \in \mathfrak{K}^{*}
$$

Отметим, что согласно следствию из леммы 12 количество гёпелевых систем, по которым происходит суммирование в (7.19) и (7.20), равно 30.

\section{§8. Модулярная природа}

До настоящего момента мы абсолютно не принимали во внимание модулярные свойства тэта-констант и их логарифмических производных. Подобная роскошь была вполне позволительной, поскольку нас интересовали только дифференциальные уравнения для этих функций. Кроме того, до сих пор оставался открытым вопрос о существовании соотношений, выражающих тэта-константы через их логарифмические производные (в случае $g=1$ таковьми являются доказанные в $\S 1$ формулы (0.8)).

Остановимся сначала на одномерном случае. Действие группы $S L_{2}(\mathbb{R})$, а также $S L_{2}(\mathbb{Z})$ и любой ее конгруэни-подгруппь

$$
\Gamma \subset\left\{\left(\begin{array}{ll}
1 & 0 \\
0 & 1
\end{array}\right)(\bmod L)\right\} \subset S L_{2}(\mathbb{Z})
$$

$($ уровня $L)$ на верхней полуплоскости $\mathfrak{H}_{1}=\{\tau: \operatorname{Im} \tau>0\}$ определяется формулой

$$
\tau \mapsto \gamma \tau=\frac{a \tau+b}{c \tau+d}, \quad \gamma=\left(\begin{array}{ll}
a & b \\
c & d
\end{array}\right) \in S L_{2}(\mathbb{R}) .
$$

Голоморфиня на $\mathfrak{H}_{1}$ функция $F(\tau)$ называется ( голоморфной) модулярной формой веса ш относительно Г, если

$$
F(\gamma \tau)=(c \tau+d)^{w} F(\tau) \quad \text { для всех } \gamma=\left(\begin{array}{ll}
a & b \\
c & d
\end{array}\right) \in \Gamma
$$


и для любого $\gamma \in S L_{2}(\mathbb{Z})$ функция $(c \tau+d)^{-w} F(\gamma \tau)$ раскладьвается в ряд Фурье

$$
\sum_{n=0}^{\infty} f_{n} e^{2 \pi i n \tau / L}
$$

Множество модулярных форм веса $w$ относительно заданной конгруэнц-подгруппы Г образует векторное пространство, которое мы обозначим через $\operatorname{Mod}_{w}(\Gamma)$. Вычисляя логарифмическую производную обеих частей равенства (8.1) для произвольной формы $F \in \operatorname{Mod}_{w}(\Gamma)$, получаем

$$
\frac{d F / d \tau}{F}\left(\frac{a \tau+b}{c \tau+d}\right)=w c(c \tau+d)+(c \tau+d)^{2} \frac{d F / d \tau}{F}\left(\frac{a \tau+b}{c \tau+d}\right), \quad \gamma=\left(\begin{array}{ll}
a & b \\
c & d
\end{array}\right) \in \Gamma
$$

Из (8.2) несложно показать, что дифференциальный оператор

$$
\mathscr{D}: F \mapsto \delta\left(\frac{\delta F}{F}\right)-\frac{1}{w}\left(\frac{\delta F}{F}\right)^{2}, \quad \delta=\frac{1}{\pi i} \frac{d}{d \tau}
$$

отображает пространство $\operatorname{Mod}_{w}(\Gamma)$ в $\operatorname{Mod}_{4}(\Gamma)$, т.е. функция $\mathscr{D} F$ является модулярной формой веса 4 относительно Г.

Для пары форм $F_{1}, F_{2} \in \operatorname{Mod}_{w}(\Gamma)$ из равенств (8.2) следует, что функция

$$
\frac{\delta F_{1}}{F_{1}}-\frac{\delta F_{2}}{F_{2}}
$$

является модулярной формой веса 2 относительно Г. Этот факт и функциональное уравнение для тэта-констант

$$
\begin{gathered}
\vartheta_{j}\left(\frac{a \tau+b}{c \tau+d}\right)=\xi(c \tau+d)^{1 / 2} \vartheta_{j}(\tau), \quad j=2,3,4, \quad \xi^{8}=1, \\
\gamma=\left(\begin{array}{ll}
a & b \\
c & d
\end{array}\right) \in \Gamma_{1,2}=\left\{\left(\begin{array}{ll}
a & b \\
c & d
\end{array}\right): a, b, c, d \in \mathbb{Z}, a d-b c=1, a b \in 2 \mathbb{Z}, c d \in 2 \mathbb{Z}\right\}
\end{gathered}
$$

(выбор $\xi=\xi(\gamma)$ и ветви квадратного корня описан в теореме 7.1 из [3; гл. I]) позволяют дать новое доказательство тождеств (0.8).

В случае $g>1$ на верхнем полупространстве Зигеля $\mathfrak{H}_{g} \subset \operatorname{Sym}_{g}(\mathbb{C})$ действует симплектическая группа

$$
S p_{2 g}(\mathbb{R})=\left\{\gamma=\left(\begin{array}{ll}
A & B \\
C & D
\end{array}\right):^{t} \gamma\left(\begin{array}{cc}
0 & E \\
-E & 0
\end{array}\right) \gamma=\left(\begin{array}{cc}
0 & E \\
-E & 0
\end{array}\right)\right\}
$$

где $A, B, C, D$ - квадратные вешественнозначные матрицы размера $g, E$ - единичная матрица. Это действие описывается формулой

$$
\mathrm{T} \mapsto \gamma \mathrm{T}=(A \mathrm{~T}+B)(C \mathrm{~T}+D)^{-1}, \quad \gamma=\left(\begin{array}{cc}
A & B \\
C & D
\end{array}\right) \in S p_{2 g}(\mathbb{R})
$$


Определение модулярной группь Зигеля $S p_{2 g}(\mathbb{Z})$ отличается от $(8.5)$ только тем, что элементы матриц $A, B, C, D$ целочисленны.

ЛЕмма 16 (см. [25; недоказанная формула (4.2)]). Пусть функция $F(\mathrm{~T})$, $F: \mathfrak{H}_{g} \rightarrow \mathbb{R}$, мероморфна, матричный дифференциальный оператор $\Delta$ определяется равенством (0.19) (т.е. $\Delta F$ - симметричная квадратная матрица размера $g$, әлементы которой - соответствующие частные $\delta_{j k}$-производные функиии F). Тогда

$$
\begin{gathered}
(\Delta F)(\gamma \mathrm{T})=(C \mathrm{~T}+D) \cdot \Delta F(\gamma \mathrm{T}) \cdot{ }^{t}(C \mathrm{~T}+D) \\
\text { для всех } \gamma=\left(\begin{array}{ll}
A & B \\
C & D
\end{array}\right) \in S p_{2 g}(\mathbb{Z}) .
\end{gathered}
$$

ДокАЗАТЕЛЬСтво. Пусть $\tau_{j k}$ и $\tau_{j k}^{\prime}, j, k=1, \ldots, g,-$ элементы матриц $\mathrm{T} \in \mathfrak{H}_{g}$ и $\mathrm{T}^{\prime}=\gamma \mathrm{T} \in \mathfrak{H}_{g}$, где $\gamma \in S p_{2 g}(\mathbb{Z})$; соответствуюшие $\delta$-дифференцирования обозначим через $\delta_{j k}$ и $\delta_{j k}^{\prime}, j, k=1, \ldots, g$. Ввиду симметричности матриц Т и Т' независимыми являются элементы с номерами $j, k, 1 \leqslant j \leqslant k \leqslant g$. Пользуясь правилом дифференцирования сложной функции, мы получаем

$$
\delta_{j k} F\left(\mathrm{~T}^{\prime}\right)=\left(\sum_{l, m=1}^{g} \frac{\partial \tau_{l m}^{\prime}}{\partial \tau_{j k}} \delta_{l m}^{\prime} F\right)\left(\mathrm{T}^{\prime}\right), \quad j, k=1, \ldots, g
$$

Действительно,

$$
\begin{aligned}
\delta_{j j} F\left(\mathrm{~T}^{\prime}\right) & =\frac{1}{\pi i} \frac{\partial F\left(\mathrm{~T}^{\prime}\right)}{\partial \tau_{j j}}=\frac{1}{\pi i}\left(\sum_{1 \leqslant l \leqslant m \leqslant g} \frac{\partial \tau_{l m}^{\prime}}{\partial \tau_{j j}} \frac{\partial F}{\partial \tau_{l m}^{\prime}}\right)\left(\mathrm{T}^{\prime}\right) \\
& =\left(\sum_{l, m=1}^{g} \frac{\partial \tau_{l m}^{\prime}}{\partial \tau_{j j}} \delta_{l m}^{\prime} F\right)\left(\mathrm{T}^{\prime}\right), \quad j=1, \ldots, g \\
\delta_{j k} F\left(\mathrm{~T}^{\prime}\right) & =\frac{1}{2 \pi i} \frac{\partial F\left(\mathrm{~T}^{\prime}\right)}{\partial \tau_{j k}}=\frac{1}{2 \pi i}\left(\sum_{1 \leqslant l \leqslant m \leqslant g} \frac{\partial \tau_{l m}^{\prime}}{\partial \tau_{j k}} \frac{\partial F}{\partial \tau_{l m}^{\prime}}\right)\left(\mathrm{T}^{\prime}\right) \\
& =\left(\sum_{l, m=1}^{g} \frac{\partial \tau_{l m}^{\prime}}{\partial \tau_{j k}} \delta_{l m}^{\prime} F\right)\left(\mathrm{T}^{\prime}\right), \quad j, k=1, \ldots, g .
\end{aligned}
$$

Равенство (8.6) достаточно проверить для образующих группы $S p_{2 g}(\mathbb{Z})$ :

$$
\gamma_{1}=\left(\begin{array}{cc}
E & B \\
0 & E
\end{array}\right), \quad \gamma_{2}=\left(\begin{array}{cc}
{ }^{t} A & 0 \\
0 & A^{-1}
\end{array}\right), \quad \gamma_{3}=\left(\begin{array}{cc}
0 & E \\
-E & 0
\end{array}\right)
$$

где матрица $A \in G L_{g}(\mathbb{Z})$ и симметричная целочисленная матрица $B$ произвольны (см. [3; гл. II, приложение к $\S 5$, предложение А.5]). С помощью прямого матричного вычисления несложно показать, что если равенство (8.6) вьполнено для $\gamma, \gamma^{\prime} \in S p_{2 g}(\mathbb{Z})$, то оно верно и для композиции $\gamma \gamma^{\prime}$.

В случае $\mathrm{T}^{\prime}=\gamma_{1} \mathrm{~T}=\mathrm{T}+B$ имеем $\tau_{j k}^{\prime}=\tau_{j k}+b_{j k}, j, k=1, \ldots, g$, откуда

$$
\delta_{j k} F\left(\mathrm{~T}^{\prime}\right)=\left(\delta_{j k} F\right)\left(\mathrm{T}^{\prime}\right), \quad j, k=1, \ldots, g .
$$


Следовательно,

$$
\Delta F\left(\gamma_{1} \mathrm{~T}\right)=(\Delta F)\left(\gamma_{1} \mathrm{~T}\right) .
$$

Если $\mathrm{T}^{\prime}=\gamma_{2} \mathrm{~T}={ }^{t} A \mathrm{~T} A$, где $A=\left(a_{j l}\right)_{j, l=1, \ldots, g}$, то

$$
\tau_{l m}^{\prime}=\sum_{j, k=1}^{g} a_{j l} a_{k m} \tau_{j k}, \quad l, m=1, \ldots, g
$$

откуда

$$
\frac{\partial \tau_{l m}^{\prime}}{\partial \tau_{j k}}=a_{j l} a_{k m}, \quad j, k=1, \ldots, g, \quad l, m=1, \ldots, g .
$$

Согласно формуле (8.7)

$$
\delta_{j k} F\left(\mathrm{~T}^{\prime}\right)=\left(\sum_{l, m=1}^{g} a_{j l} a_{k m} \delta_{l m}^{\prime} F\right)\left(\mathrm{T}^{\prime}\right), \quad j, k=1, \ldots, g,
$$

значит

$$
\Delta F\left(\gamma_{2} \mathrm{~T}\right)=A \cdot(\Delta F)\left(\gamma_{2} \mathrm{~T}\right) \cdot{ }^{t} A .
$$

Для последнего образующего элемента $\gamma_{3}$ имеем $\mathrm{T}^{\prime}=\gamma_{3} \mathrm{~T}=-\mathrm{T}^{-1}$, иными словами, $\mathrm{T}^{\prime} \mathrm{T}=-E$. В этом случае

$$
0=\frac{\partial}{\partial \tau_{j k}}\left(\mathrm{~T}^{\prime} \mathrm{T}\right)=\frac{\partial}{\partial \tau_{j k}} \mathrm{~T}^{\prime} \cdot \mathrm{T}+\mathrm{T}^{\prime} \cdot \frac{\partial}{\partial \tau_{j k}} \mathrm{~T}, \quad j, k=1, \ldots, g
$$

(в левой части стоит нулевая матрица), откуда

$$
\frac{\partial}{\partial \tau_{j k}} \mathrm{~T}^{\prime}=-\mathrm{T}^{\prime} \cdot \frac{\partial}{\partial \tau_{j k}} \mathrm{~T} \cdot \mathrm{T}^{-1}=\mathrm{T}^{\prime} \cdot \frac{\partial}{\partial \tau_{j k}} \mathrm{~T} \cdot \mathrm{T}^{\prime}, \quad j, k=1, \ldots, g,
$$

и, следовательно,

$$
\frac{\partial \tau_{l m}^{\prime}}{\partial \tau_{j k}}=\tau_{l j}^{\prime} \tau_{k m}^{\prime}, \quad j, k=1, \ldots, g, \quad l, m=1, \ldots, g
$$

Подставляя полученный результат в соотношения (8.7), находим

$$
\delta_{j k} F\left(\mathrm{~T}^{\prime}\right)=\left(\sum_{l, m=1}^{g} \tau_{l j}^{\prime} \tau_{k m}^{\prime} \delta_{l m}^{\prime} F\right)\left(\mathrm{T}^{\prime}\right), \quad j, k=1, \ldots, g,
$$

значит

$$
\Delta F\left(\gamma_{3} \mathrm{~T}\right)=\mathrm{T}^{\prime} \cdot(\Delta F)\left(\gamma_{3} \mathrm{~T}\right) \cdot \mathrm{T}^{\prime}=\mathrm{T}^{-1} \cdot(\Delta F)\left(\gamma_{3} \mathrm{~T}\right) \cdot \mathrm{T}^{-1} .
$$

Собирая вместе равенства (8.9)-(8.11), заключаем, что

$$
\Delta F(\gamma \mathrm{T})=(C \mathrm{~T}+D)^{-1} \cdot(\Delta F)(\gamma \mathrm{T}) \cdot{ }^{t}(C \mathrm{~T}+D)^{-1}
$$

(матрица Т симметрична) для образуюших элементов (8.8) групшы $S p_{2 g}(\mathbb{Z}) . \mathrm{Pa}$ венство (8.6) следует из (8.12). Таким образом, равенство (8.6) справедливо для всех $\gamma \in S p_{2 g}(\mathbb{Z})$. Лемма доказана. 
Голоморфная (мероморфная) на $\mathfrak{H}_{g}$ функция $F(\mathrm{~T})$ назьвается голоморфной (соответственно мероморфной) модулярной формой веса ш относительно конгруэнц-подгруппы

$$
\Gamma \subset\left\{\left(\begin{array}{cc}
E & 0 \\
0 & E
\end{array}\right)(\bmod L)\right\} \subset S p_{2 g}(\mathbb{Z})
$$

если

$$
F(\gamma \mathrm{T})=\operatorname{det}^{w}(C \mathrm{~T}+D) \cdot F(\mathrm{~T}) \quad \text { для всех } \gamma=\left(\begin{array}{cc}
A & B \\
C & D
\end{array}\right) \in \Gamma
$$

Дополнительные условия, ограничивающие рост функции $F(\mathrm{~T})$ в “вершинах", при $g>1$ выполняются автоматически - это так называемый принщип Кёхера (см. [2; гл. V, лемма 19]).

В полном соответствии с одномерным случаем множество модулярных форм веca $w$ относительно заданной конгруэнщ-подгруппы Г образует векторное пространство, которое мы обозначаем через $\operatorname{Mod}_{w}(\Gamma)=\operatorname{Mod}_{w}^{(g)}(\Gamma)$.

Лемма 17. Пусть $F: \mathfrak{H}_{g} \rightarrow \mathbb{R}$ - модулярная форма веса ш относительно конгруәни-подгруппь $\Gamma \subset S p_{2 g}(\mathbb{Z})$. Тогда (матричнозначная) функиия

$$
\Psi=\frac{\Delta F}{F}: \mathfrak{H}_{g} \rightarrow \operatorname{Sym}_{g}(\mathbb{C})
$$

удовлетворяет функииональному уравнению

$$
\Psi(\gamma \mathrm{T})=\pi i w(C \mathrm{~T}+D) \cdot{ }^{t} C+(C \mathrm{~T}+D) \cdot \Psi(\mathrm{T}) \cdot{ }^{t}(C \mathrm{~T}+D), \quad \gamma \in \Gamma .
$$

ДокАЗАТЕЛьство. Отметим сразу несложное равенство

$$
\frac{\Delta \operatorname{det}(C \mathrm{~T}+D)}{\operatorname{det}(C \mathrm{~T}+D)}=\pi i^{t} C \cdot{ }^{t}(C \mathrm{~T}+D)^{-1}
$$

для произвольных матриц $C, D$. Проверяется оно непосредственным вычислением (ср. с [25; формула (4.3)]).

Логарифмируя равенства (8.13) и применяя дифференциальньй оператор $\Delta, \mathrm{c}$ учетом (8.16) получим

$$
\frac{\Delta F(\gamma \mathrm{T})}{F(\gamma \mathrm{T})}=\pi i w^{t} C \cdot{ }^{t}(C \mathrm{~T}+D)^{-1}+\frac{\Delta F(\mathrm{~T})}{F(\mathrm{~T})}, \quad \gamma \in \Gamma,
$$

откуда

$$
\begin{aligned}
& (C \mathrm{~T}+D) \cdot \frac{\Delta F(\gamma \mathrm{T})}{F(\gamma \mathrm{T})} \cdot{ }^{t}(C \mathrm{~T}+D) \\
& \quad=\pi i w(C \mathrm{~T}+D) \cdot{ }^{t} C+(C \mathrm{~T}+D) \cdot \frac{\Delta F(\mathrm{~T})}{F(\mathrm{~T})} \cdot{ }^{t}(C \mathrm{~T}+D), \quad \gamma \in \Gamma .
\end{aligned}
$$

Согласно лемме 16

$$
(C \mathrm{~T}+D) \cdot \frac{\Delta F(\gamma \mathrm{T})}{F(\gamma \mathrm{T})} \cdot{ }^{t}(C \mathrm{~T}+D)=\left(\frac{\Delta F}{F}\right)(\gamma \mathrm{T}), \quad \gamma \in S p_{2 g}(\mathbb{Z})
$$

Сравнивая соотношения (8.17) и (8.18), получаем (8.15). 
СЛЕДСТВИЕ 1. Пусть $F_{1}$ и $F_{2}$ - произвольные модулярные форми веса $ш$ относительно конгруэни-подгруппь $\Gamma \subset S p_{2 g}(\mathbb{Z})$. Тогда функиия

$$
\operatorname{det}\left(\frac{\Delta F_{1}}{F_{1}}-\frac{\Delta F_{2}}{F_{2}}\right)(\mathrm{T})
$$

является мероморфной модулярной формой веса 2 относительно той жсе самой подгруппь $Г$.

ДокАЗАТЕльство. Применяя лемму 17 к функциям $F_{1}, F_{2}$, вычитая одно получающееся равенство из другого и вычисляя определители, находим

$$
\operatorname{det}\left(\frac{\Delta F_{1}}{F_{1}}-\frac{\Delta F_{2}}{F_{2}}\right)(\gamma \mathrm{T})=\operatorname{det}^{2}(C \mathrm{~T}+D) \cdot \operatorname{det}\left(\frac{\Delta F_{1}}{F_{1}}-\frac{\Delta F_{2}}{F_{2}}\right)(\mathrm{T}), \quad \gamma \in \Gamma
$$

Последнее соотношение в точности означает, что функция (8.19) является модулярной формой веса 2 относительно конгруэнц-подгруппы $Г$.

ЗАМЕчАнИЕ. В отличие от случая $g=1$ элементы матричнозначной функции (8.14) не являются голоморфными на $\mathfrak{H}_{g}$ функциями, поэтому мы вынуждены подчеркнуть, что функция (8.19) является мероморфной модулярной формой. В случае голоморфных модулярных форм $F_{1}, F_{2}$ их $\Delta$-производные состоят из голоморфных функций, значит мы можем уточнить последний результат следующим образом.

СлЕДСТВИЕ 2. Пусть $F_{1}$ и $F_{2}$ - произвольные голоморфные модулярные формы веса $w$ относительно конгруәни-подгруппь $\Gamma \subset S p_{2 g}(\mathbb{Z})$. Тогда функиия

$$
\left(F_{1} F_{2}\right)^{g} \cdot \operatorname{det}\left(\frac{\Delta F_{1}}{F_{1}}-\frac{\Delta F_{2}}{F_{2}}\right)(\mathrm{T})
$$

является голоморфной модулярной формой веса $2 w g+2$ относительно той жее самой подгруппь $\Gamma$.

ДоКАЗАТЕЛЬСТво состоит в подсчете веса голоморфной модулярной формы (8.20), так как ее модулярность получается из следствия 1.

Следствия из леммы 17 в некотором смысле обобщают дифференциальную операцию (8.4) на случай произвольной размерности. Отметим, что диффференциальный оператор (8.3) также имеет многомерные обобщения (см. [26]).

Как и в одномерном случае, при $g>1$ сушествует функциональное уравнение, связьвающее любую из тэта-констант:

$$
\begin{gathered}
\vartheta_{\boldsymbol{a}}\left((A \mathrm{~T}+B)(C \mathrm{~T}+D)^{-1}\right)=\xi \cdot \operatorname{det}^{1 / 2}(C \mathrm{~T}+D) \cdot \vartheta_{\boldsymbol{a}}(\mathrm{T}), \quad \boldsymbol{a} \in \mathfrak{K}^{*}, \quad \xi^{8}=1, \\
\left(\begin{array}{cc}
A & B \\
C & D
\end{array}\right) \in \Gamma_{1,2}=\Gamma_{1,2}^{(g)}
\end{gathered}
$$

(см. [3; гл. II, $\S 5])$. Конгруэнщ-подгруппа $\Gamma_{1,2} \subset S p_{2 g}(\mathbb{Z})$ (уровня 2 ) порождается элементами (8.8), где выбор матрищы $A \in G L_{g}(\mathbb{Z})$ и симметричной целочисленной 
матрицы $B$ с четной главной диагональю произволен (см. [3; гл. II, приложение к $\S 5$, предложение А.4]). Определение (8.13) модулярных форм можно расширить, допуская появление корней из единицы в качестве коэффициентов в правой части (8.13). Не заостряя на этом внимание, отметим, что наличие постоянного множителя $\xi$ не влияет на логарифмическое $\Delta$-дифференцирование уравнения (8.21), поэтому согласно лемме 17 мы получаем следуюшее утверждение.

Лемма 18. Для любой четной характеристики а матричнозначная функиия

$$
\Psi_{\boldsymbol{a}}=\frac{\Delta \vartheta_{\boldsymbol{a}}}{\vartheta_{\boldsymbol{a}}}: \mathfrak{H}_{g} \rightarrow \operatorname{Sym}_{g}(\mathbb{C}),
$$

составленная из логарифмических производных тэта-констант (см. формуль (0.16)), удовлетворяет функциональному уравнению

$$
\Psi_{\boldsymbol{a}}(\gamma \mathrm{T})=\frac{\pi i}{2}(C \mathrm{~T}+D) \cdot{ }^{t} C+(C \mathrm{~T}+D) \cdot \Psi_{\boldsymbol{a}}(\mathrm{T}) \cdot{ }^{t}(C \mathrm{~T}+D), \quad \gamma \in \Gamma_{1,2}
$$

СлЕДСТвИЕ 1. Для любых четных характеристик $\boldsymbol{a}, \boldsymbol{b} \in \mathfrak{K}$ функция

$$
\left(\vartheta_{\boldsymbol{a}} \vartheta_{\boldsymbol{b}}\right)^{g} \cdot \operatorname{det}\left(\Psi_{\boldsymbol{a}}-\Psi_{\boldsymbol{b}}\right)(\mathrm{T})
$$

является голоморфной модулярной формой веса $g+2$ относительно $\Gamma_{1,2}$.

Разумеется, следствие 1 является только одним следствием из леммы 18. Приведем более общий результат в духе $\S 7$.

СлЕДСТВИЕ 2. Пусть аддитивная система характеристик $\mathfrak{G}$ в $\mathfrak{K}$ размерности $l \leqslant g$ разбита на две непересекающиеся аддитивные подсистемы $\mathfrak{F}+\boldsymbol{c}$ u $\mathfrak{F}$ размерности $l-1: \mathfrak{G}=(\mathfrak{F}+\boldsymbol{c}) \cup \mathfrak{F}$, где $\boldsymbol{c} \in \mathfrak{K}-$ ненулевая характеристика из аддитивной группы, соответствующей $\mathfrak{G}$. Тогда функиия

$$
\left(\prod_{\boldsymbol{a} \in \mathfrak{F}} \vartheta_{\boldsymbol{a}+\boldsymbol{c}} \vartheta_{\boldsymbol{a}}\right)^{g} \cdot \operatorname{det}\left(\sum_{\boldsymbol{a} \in \mathfrak{F}}\left(\Psi_{\boldsymbol{a}+\boldsymbol{c}}-\Psi_{\boldsymbol{a}}\right)\right)
$$

является голоморфной модулярной формой веса $2^{l-1} g+2$ относительно $\Gamma_{1,2}$.

В случаях $g=2$ и $g=3$ этим результатам удается придать количественную форму. Для произвольного подмножества $\mathfrak{G} \subset \mathfrak{K}^{*}$ введем обозначения

$$
\vartheta_{\mathfrak{G}}=\prod_{\boldsymbol{a} \in \mathfrak{G}} \vartheta_{\boldsymbol{a}}, \quad \Psi_{\mathfrak{G}}=\sum_{\boldsymbol{a} \in \mathfrak{G}} \Psi_{\boldsymbol{a}}
$$

Положим

$$
\chi_{g}= \begin{cases}\vartheta_{\mathfrak{K}^{*}}^{4} & \text { при } g=1, \\ \vartheta_{\mathfrak{K}^{*}}^{2} & \text { при } g=2, \\ \vartheta_{\mathfrak{K}^{*}} & \text { при } g=3\end{cases}
$$

(минимальные параболические модулярные формы относительно $S p_{2 g}(\mathbb{Z})$ в размерностях 1, 2 и 3 соответственно, см. [15]). 
Лемма 19. Пусть $g \leqslant 3$ и гёпелева система характеристик $\mathfrak{G}$ в $\mathfrak{K}$ разбита на две непересекающиеся аддитивные подсистемы $\mathfrak{F}+\boldsymbol{c}$ и $\mathfrak{F}$ размерности $g-1$. Тогда

$$
\operatorname{det}\left(\Psi_{\mathfrak{F}+\boldsymbol{c}}-\Psi_{\mathfrak{F}}\right)= \pm \frac{1}{2^{2 g}} \frac{\chi_{g}}{\left(\vartheta_{\mathfrak{F}+\boldsymbol{c}} \vartheta_{\mathfrak{F}}\right)^{4}} .
$$

ДокАЗАТЕЛЬСтво. При $g=1$ соотношения (8.24) совпадают с формулами (0.8) (в последних, правда, знак “土” конкретизирован). Согласно основному результату $\S 5$ из [2] голоморфные формы (8.22) могут быть представлены как многочлены от квадратов тэта-констант при $g=2$ и от тэта-констант при $g=3$. Поэтому доказательство сводится к вычислению первых коэффициентов разложений Фурье для голоморфных функций (8.22), а также для мономов от квадратов тэта-констант степени 6 при $g=2$ и мономов от тэта-констант степени 28 при $g=3$. Детали этих вычислений остаются за пределами данной работы.

Теорема 6. При $g \leqslant 3$ тэта-константы алгебраичны над полем, порожденным их $\delta$-логарифмическими производными (0.16).

ДокАЗАТЕльство. В случае $g=1$ согласно формулам (0.8) четвертые степени тэта-констант являются (с точностью до постоянного множителя) разностями $\delta$-логарифмических производных $(0.5)$.

Формулы (8.24) не зависят (с точностью до знака) от разбиения гёпелевой системы $\mathfrak{G}=(\mathfrak{F}+\boldsymbol{c}) \cup \mathfrak{F}$ на две непересекающиеся аддитивные системы. Поэтому мы зафиксируем для каждой гёпелевой системы одно из таких разбиений, положив

$$
\lambda_{\mathfrak{G}}=\operatorname{det}\left(\Psi_{\mathfrak{F}+\boldsymbol{c}}-\Psi_{\mathfrak{F}}\right),
$$

и перепишем соотношения (8.24) в виде

$$
\pm 2^{2 g} \lambda_{\mathfrak{G}}=\chi_{g} \vartheta_{\mathfrak{G}}^{-4}
$$

Перемножая равенства (8.26) по всем гёпелевым системам в $\mathfrak{K}$, число которых согласно лемме 12 равно 15 при $g=2$ и 135 при $g=3$, мы получим

$$
\begin{array}{ccc} 
\pm 2^{60} \prod_{\mathfrak{G}} \lambda_{\mathfrak{G}} & =\chi_{2}^{15} \prod_{\mathfrak{G}} \vartheta_{\mathfrak{G}}^{-4}=\chi_{2}^{15} \prod_{\boldsymbol{a} \in \mathfrak{K}^{*}} \vartheta_{\boldsymbol{a}}^{-24}=\chi_{2}^{3} \quad \text { при } g=2, \\
\pm 2^{6 \cdot 135} \prod_{\mathfrak{G}} \lambda_{\mathfrak{G}} & =\chi_{3}^{135} \prod_{\mathfrak{G}} \vartheta_{\mathfrak{G}}^{-4}=\chi_{3}^{135} \prod_{\boldsymbol{a} \in \mathfrak{K}^{*}} \vartheta_{\boldsymbol{a}}^{-120}=\chi_{3}^{15} & \text { при } g=3 .
\end{array}
$$

Мы воспользовались определением (8.23) величин $\chi_{2}, \chi_{3}$ и следствием из леммы 12: каждая четная характеристика участвует в 6 гёпелевых системах при $g=2$ и в 30 системах - при $g=3$.

Фиксируя четную характеристику $\boldsymbol{a}$, перемножим равенства (8.26) по всем гёпелевьм системам $\mathfrak{G}$, содержащим $\boldsymbol{a}$ :

$$
\begin{aligned}
& \pm 2^{24} \prod_{\mathfrak{G} \ni \boldsymbol{a}} \lambda_{\mathfrak{G}}=\chi_{2}^{6} \prod_{\mathfrak{G} \ni \boldsymbol{a}} \vartheta_{\mathfrak{G}}^{-4}=\chi_{2}^{6} \cdot \vartheta_{\boldsymbol{a}}^{-16} \prod_{\boldsymbol{b} \in \mathfrak{K}^{*}} \vartheta_{\boldsymbol{b}}^{-8}=\chi_{2}^{2} \cdot \vartheta_{\boldsymbol{a}}^{-16} \quad \text { при } g=2 \\
& \pm 2^{6 \cdot 30} \prod_{\mathfrak{G} \ni \boldsymbol{a}} \lambda_{\mathfrak{G}}=\chi_{3}^{30} \prod_{\mathfrak{G} \ni \boldsymbol{a}} \vartheta_{\mathfrak{G}}^{-4}=\chi_{3}^{30} \cdot \vartheta_{\boldsymbol{a}}^{-96} \prod_{\boldsymbol{b} \in \mathfrak{K}^{*}} \vartheta_{\boldsymbol{b}}^{-24}=\chi_{3}^{6} \cdot \vartheta_{\boldsymbol{a}}^{-96} \quad \text { при } g=3 .
\end{aligned}
$$


Возводя равенства (8.28) в куб, (8.29) в пятую степень и подставляя в них соотношения (8.27), находим

$$
\begin{aligned}
\vartheta_{\boldsymbol{a}}^{48} & = \pm \chi_{2}^{6} \cdot 2^{72} \prod_{\mathfrak{G} \ni \boldsymbol{a}} \lambda_{\mathfrak{G}}^{-3}= \pm 2^{120} \prod_{\mathfrak{G}} \lambda_{\mathfrak{G}}^{2} \cdot 2^{72} \prod_{\mathfrak{G} \ni \boldsymbol{a}} \lambda_{\mathfrak{G}}^{-3} \\
& = \pm 2^{192} \prod_{\mathfrak{G} \not \boldsymbol{a}} \lambda_{\mathfrak{G}}^{2} \cdot \prod_{\mathfrak{G} \ni \boldsymbol{a}} \lambda_{\mathfrak{G}}^{-1} \quad \text { при } g=2, \\
\vartheta_{\boldsymbol{a}}^{480} & = \pm \chi_{2}^{30} \cdot 2^{900} \prod_{\mathfrak{G} \ni \boldsymbol{a}} \lambda_{\mathfrak{G}}^{-5}= \pm 2^{12 \cdot 135} \prod_{\mathfrak{G}} \lambda_{\mathfrak{G}}^{2} \cdot 2^{900} \prod_{\mathfrak{G} \ni \boldsymbol{a}} \lambda_{\mathfrak{G}}^{-5} \\
& = \pm 2^{2520} \prod_{\mathfrak{G} \not \boldsymbol{a}} \lambda_{\mathfrak{G}}^{2} \cdot \prod_{\mathfrak{G} \ni \boldsymbol{a}} \lambda_{\mathfrak{G}}^{-3} \quad \text { при } g=3 .
\end{aligned}
$$

Соотношения (8.30) означают, что при $g=2$ и $g=3$ тэта-константы алгебраичны над полем, порожденным функциями (8.24), которые являются многочленами от логарифмических производных (0.16) тэта-констант. Теорема доказана.

И (8.24), и (8.30) можно рассматривать как обобщения формул (0.8) на случаи $g=2$ и $g=3$. Отметим также подобные формулы, доказательство которых "совпадает" с доказательством леммы 19, и поэтому мы опускаем его.

Лемма 20. а) Пусть $g=2$. Тогда для любих четных характеристик $\boldsymbol{a}, \boldsymbol{b}$ справедливо соотношение

$$
\operatorname{det}\left(\Psi_{\boldsymbol{a}}-\Psi_{\boldsymbol{b}}\right)= \pm \frac{1}{2^{4}} \chi_{2} \cdot \prod_{\mathfrak{G} \supset\{\boldsymbol{a}, \boldsymbol{b}\}} \vartheta_{\mathfrak{G}}^{-2},
$$

где произведение $\prod_{\mathfrak{G} \supset\{\boldsymbol{a}, \boldsymbol{b}\}}$ берется по всем (двум) гёпелевым системам, содержащим характеристики $\boldsymbol{a}, \boldsymbol{b}$.

б) Пусть $g=3$ и четные характеристики $\boldsymbol{a}_{1}, \boldsymbol{a}_{2}, \boldsymbol{a}_{3}, \boldsymbol{a}_{4}$ образуют аддитивную систему $\mathfrak{A}$ (размерности 2). Тогда

$$
\operatorname{det}\left(\Psi_{\boldsymbol{a}_{1}}+\Psi_{\boldsymbol{a}_{2}}-\Psi_{\boldsymbol{a}_{3}}-\Psi_{\boldsymbol{a}_{4}}\right)= \pm \frac{1}{2^{6}} \chi_{3} \cdot \prod_{\mathfrak{G} \supset \mathfrak{A}} \vartheta_{\mathfrak{G}}^{-2},
$$

где произведение $\prod_{\mathfrak{G} \supset \mathfrak{A}}$ берется по всем (двум) гёпелевым системам, содержащим данную систему $\mathfrak{A}$.

\section{Список литературы}

1. Krazer A. Lehrbuch der Thetafunktionen. Leipzig, 1903; Reprint. New York: Chelsea Publ., 1970.

2. Igusa J. Theta functions. Grundlehren Math. Wiss. V. 194. Berlin-Heidelberg-New York: Springer-Verlag, 1972.

3. Мамфорд Д. Лекции о тэта-функциях. М.: Мир, 1988.

4. Jacobi C. G. J. Fundamenta nova theoriae functionum ellipticarum. Regiomonti: Sumtibus Fratrum Borntraeger, 1829; // Gesammelte Werke. V. 1. Berlin: Akademie der Wissenschaften, 1881. P. 49-239; // Reprint. New York: Chelsea Publ., 1969.

5. Jacobi C. G. J. Theorie der elliptischen Functionen aus den Eigenschaften der Thetareihen abgeleitet // Gesammelte Werke. V. 1. Berlin: Akademie der Wissenschaften, 1881. P. 497-538; // Reprint. New York: Chelsea Publ., 1969.

6. Уиттекер Э. Т., Ватсон Дж. Н. Курс современного анализа. Т. 2. М.: Физматлит, 1963. 
7. Jacobi C. G. J. Über die Differentialgleichung, welcher die Reihen $1 \pm 2 q+2 q^{4} \pm 2 q^{9}+$ etc., $2 \sqrt[4]{q}+2 \sqrt[4]{q^{9}}+2 \sqrt[4]{q^{25}}+$ etc. Genüge leisten // Crelle's J. für Math. 1848. V. 36. № 2 . P. 97-112; // Gesammelte Werke. V. 2. Berlin: Akademie der Wissenschaften, 1882. P. 171-190; // Reprint. New York: Chelsea Publ., 1969.

8. Bertrand D. Formes modulaires et transcendance. Notes de cours du Centre Émile Borel. V. 17. Paris: Institut Henri Poincaré, 1999.

9. Mahler $K$. On algebraic differential equations satisfied by automorphic functions // J. Austral. Math. Soc. 1969. V. 10. P. 445-450.

10. Bertrand $D$. Theta functions and transcendence. International symposium on number theory (Madras, 1996) // Ramanujan J. 1997. V. 1. № 4. P. 339-350.

11. Bertrand D. Chapter $1 . \Theta(\tau, z)$ and transcendence // Introduction to algebraic independence theory / ed. Yu. V. Nesterenko, P. Philippon. Indépendance algébrique (Luminy, 1997). Berlin: Springer-Verlag, 2000. P. 1-11.

12. Halphen $M$. Sur un système d'équations différentielles // C. R. Acad. Sci. Paris. 1881. V. 92. P. 1101-1103.

13. Radimacher $H$. Topics in analytic number theory. Grundlehren Math. Wiss. V. 169. Berlin-Heidelberg-New York: Springer-Verlag, 1973.

14. Ramanujan S. On certain arithmetical functions // Trans. Cambridge Philosoph. Soc. 1916. V. 22. № 9. P. 159-184; // Collected papers / ed. G. H. Hardy, P. V. Sechu Aiyar, B. M. Wilson. Cambridge: Cambridge Univ. Press, 1927. P. 136-162; // Reprint. New York: Chelsea Publ., 1962.

15. Freitag E. Siegelsche Modulfunktionen. Berlin-Heidelberg: Springer-Verlag, 1983.

16. Lawden D. F. Elliptic functions and applications. Appl. Math. Sci. V. 80. Springer: New York-Berlin, 1989.

17. Нестеренко Ю. В. Модулярные функции и вопросы трансцендентности // Матем. сб. 1996. Т. 187. №9. С. 65-96.

18. Нестеренко Ю. В. О мере алгебраической независимости значений функций Рамануджана // Труды МИАН. 1997. Т. 218. С. 299-334.

19. Нестеренко Ю.В. Оценки кратностей нулей для тэта-констант // Теоретическая и прикладная матем. 1999. Т. 5. № 1. С. 1-6.

20. Ohyama $Y$. Systems of nonlinear differential equations related to second order linear equations // Osaka J. Math. 1996. V. 33. P. 927-949.

21. Bertrand D., Zudilin W. On the transendence degree of the differential field generated by Siegel modular forms // Prépubl. de l'Institut de Math. de Jussieu. V. 248, 2000; // http://xxx.lanl.gov/abs/math/0006176.

22. Ohyama Y. Differential equations of theta constants of genus two // Algebraic Analysis of Singular Perturbations (in Japanese) (Kyoto, 1996). Sūrikaisekikenkyūsho Kōkyūroku. V. 968. Kyoto: Kyoto Univ., 1996. P. 96-103; Preprint. Osaka: Osaka Univ., 1996.

23. Ohyama Y. Nonlinear equations on theta constants of genus two. Preprint. Osaka: Osaka Univ., 1996.

24. Adin R. M., Kopeliovich Ya. Short eigenvectors and multidimensional theta functions // Linear Algebra Appl. 1997. V. 257. P. 49-63.

25. Shimura G. On the derivatives of theta functions and modular forms // Duke Math. J. 1977. V. 44. P. 365-387.

26. Resnikoff H. L. Automorphic forms and automorphy preserving differential operators on products of halfplanes // Abh. Math. Sem. Univ. Hamburg. 1972. V. 38. P. 168-198.

Московский государственнњй

Поступила в редакцию

университет им. М.В. Ломоносова

19.11.1999

E-mail: wadim@ips.ras.ru 